\title{
Aeromagnetic surveying with unmanned aircraft systems
}

\author{
by \\ Michael Cunningham \\ B.Sc. (Hons.) Physics, 2010 \\ B.Sc. (Hons.) Earth Sciences, 2014
}

A thesis submitted to the Faculty of Graduate and Postdoctoral Affairs in partial fulfillment for the degree of

\author{
Master of Science \\ in \\ Earth Sciences \\ Carleton University \\ Ottawa, Ontario
}

(C)2016

Michael Cunningham 


\section{Abstract}

UASs have been under rapid development for applications in the mineral exploration industry, mainly for aeromagnetic surveying. They provide improved detection of smaller, deeper and weakly magnetic targets. A traditional system flying an altitude of $100 \mathrm{~m}$ above ground level can detect a spherical ore body at a depth of $40 \mathrm{~m}$ and magnetic susceptibility of $10^{-4}$, with a radius of $\sim 16 \mathrm{~m}$. A UAS flying at $50 \mathrm{~m}$ or $2 \mathrm{~m}$ altitude AGL would require the radius to be $11 \mathrm{~m}$ or $5 \mathrm{~m}$, respectively. Using the Venturer fixed-wing UAS and the SkyLance rotary-wing UAS, two aeromagnetic surveys were performed. The Venturer was stable in flight with a fourth difference noise level within industry standards of $\pm 0.05 \mathrm{nT}$. A magnetic map was produced showing local variations. The SkyLance flew over a zinc deposit with 3 magnetic anomalies. It produced repeatable data that compared well with upward continuation maps of ground magnetic data. 


\section{Statement of Originality}

I certify that the material presented in this thesis is my own work.

Chapter 3 - UAS Capabilities, is based on a paper submitted to Unmanned System Canada for their student papers competition (Cunningham and Samson, 2015). I am the first author of this paper which was later presented at the Unmanned Systems Canada Conference. The material within this chapter is fully my own work. The analysis was performed using tools developed by myself within MATLAB.

Chapter 4 - Claresholm, AB, is an aeromagnetic survey performed by the Venturer fixed-wing UAS. This material is from an article that is presently in press in the journal The Leading Edge entitled "Experimental aeromagnetic survey using an unmanned air system" (Wood, A., et al., 2016). The actual aeromagnetic survey was performed prior to my starting a M.Sc., so I was not involved with data acquisition. However, I was solely responsible for the geophysical analysis of the data. I developed tools in MATLAB and performed the data corrections, such as the diurnal correction and tie-line levelling. Further analysis on flight stability was performed by myself.

Chapter 5 - Nash Creek, NB describes ground and airborne magnetic surveys. The ground magnetic survey was planned and executed by myself. Andrew Hay served as my field assistant during the survey. I was responsible for data analysis using tools developed by myself in MATLAB. The aeromagnetic survey was planned by me. I further assisted the Stratus Aeronautics team, Alan Wood (hardware engineer and pilot) and Ian Cook (computer scientist), with the setup of the SkyLance 
rotary-wing UAS. Data analysis was performed by myself with tools developed in MATLAB. 


\section{Acknowledgements}

I would first like to enormously thank my supervisor, Dr. Claire Samson, for her guidance, expertise, and patience. Dr. Samson helped ensure this project was successful as well as enjoyable. She took time out of her busy schedule to not only provide guidance, but to also assist at the Nash Creek, New Brunswick, property during the SkyLance UAS survey; driving both ways from Ottawa to Nash Creek, New Brunswick in order to ensure we were there.

I also sincerely thank the Stratus Aeronautics team: Alan Wood, Ian Cook, Buddy Doyle, Doug Pollitt, and David Rutt; for without them this M.Sc. project would not have been possible. It was a pleasure to have the opportunity to visit the office in Vancouver, British Columbia; to meet with everyone and have my questions answered as well as to discuss both the Venturer and SkyLance UASs in detail. A key to this projects success was Stratus Aeronautics openness and dedication through their sharing of the aeromagnetic survey data from the Venturer UAS near Claresholm, Alberta, and putting in the long days and late nights in order to make sure the SkyLance UAS was ready to fly. Most importantly, I thank them for prioritizing my research and coming to the Nash Creek, New Brunswick property during October 2015 to perform an aeromagnetic survey with the SkyLance UAS.

A big thanks goes out to Dr. Bernd Milkereit, from the University of Toronto, for inviting me to join him and his students in early August, 2015, to visit the Nash Creek, New Brunswick property, and for allowing me use of his ground magnetic survey equipment in order to survey the full property. Furthermore, for taking the 
time to discuss the geology of the property in detail to help ensure the survey and this project were successful.

To Andrew Hay, a sincere thanks for taking the time in August, 2015 to drive us both to Nash Creek, New Brunswick, and for assisting with the ground magnetic survey over the property. Thanks for also providing insight into magnetic surveying methods and techniques, as well as being someone whom I could discuss results and data analysis methods with.

I would also like to thank my fellow students, Kyle Harris, Peter Oliver, and Jennifer Blanchard for having productive discussions about my analysis methods as well as providing insight into how I could solve specific problems. Thanks to Jennifer for providing me with access to geophysical analysis software (Geosoft's Oasis Montaj) to perform upward continuation analysis on the Nash Creek, New Brunswick, ground magnetic data.

Finally, a heartfelt thanks to my partner, Trisha Payne, for supporting me during this project; for putting in many hours reading over my papers, repeatedly listening to my presentations, and ensuring I stay on task to meet my many deadlines. Without you, I would never have gotten through this project. 


\section{Table of Contents}

Abstract ................................................................................................................................................

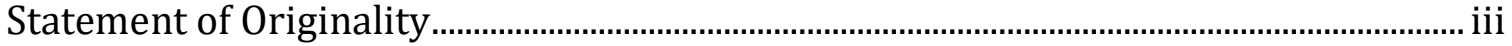

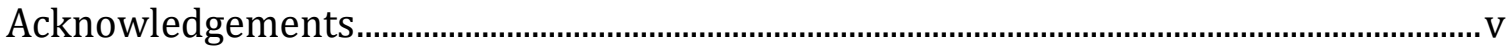

Table of Contents ...........................................................................................................................vii

List of Tables .......................................................................................................................ix

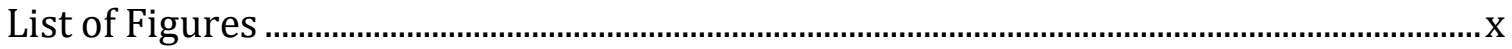

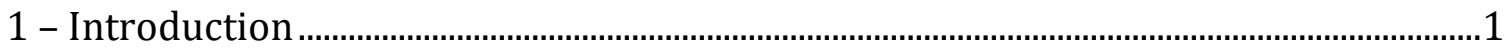

1.1 - Research Objectives ............................................................................................

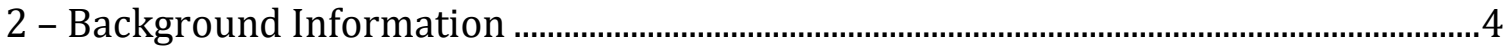

2.1 - Magnetic Methods..........................................................................................................

2.1.1 - Magnetic Induction Principles............................................................................9

2.1.2 - Total Magnetic Intensity ....................................................................................11

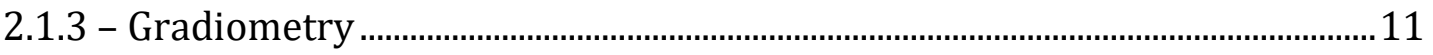

2.1.4 - Magnetometers ..........................................................................................................12

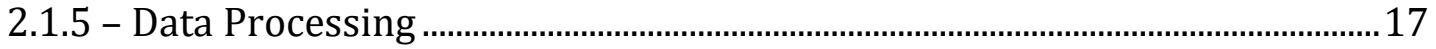

2.2 - Unmanned Aircraft Survey Systems ……………………………………………....23

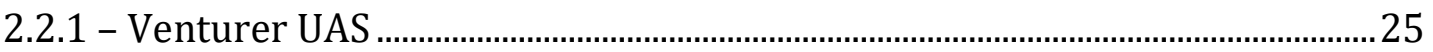

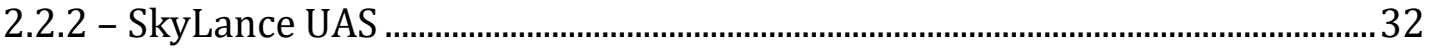

3 - UAS Theoretical Capabilities...............................................................................................

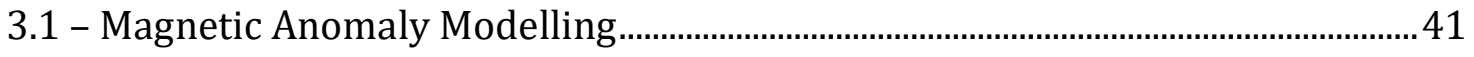

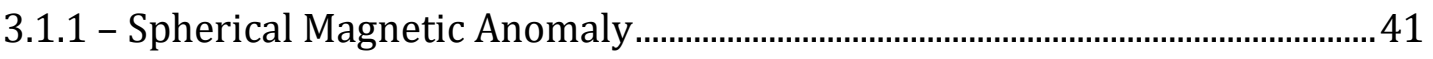

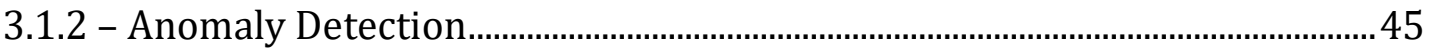

3.1.3 - Gradiometry Capabilities ......................................................................................52

3.1.4 - Spatial Resolution .................................................................................................56

3.2 - Results .............................................................................................................

3.2.1 - Minimum Detection Limits ..............................................................................61

3.2.2 - Gradiometry Capabilities .....................................................................................66

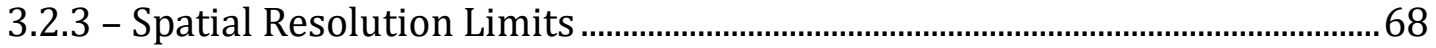

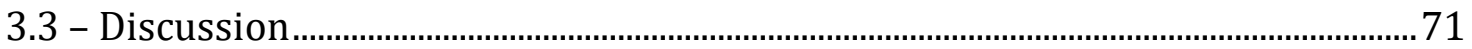

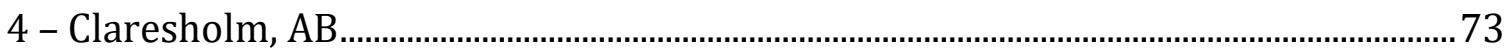

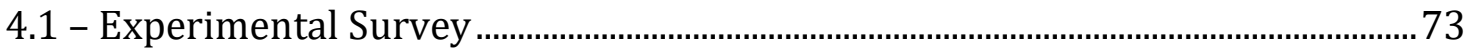




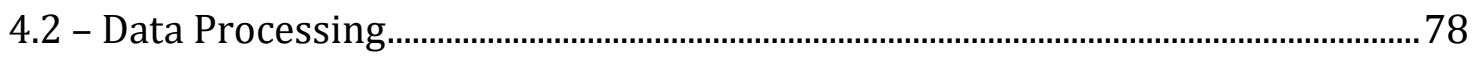

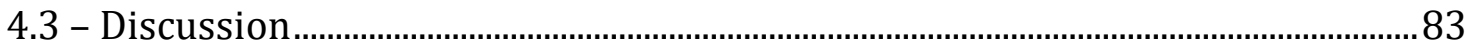

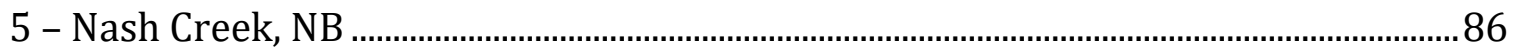

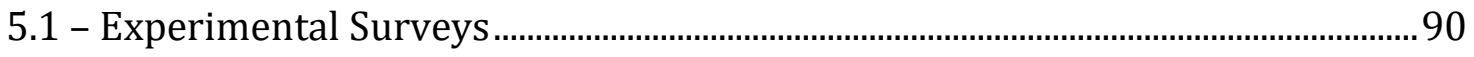

5.1.1 - Ground Magnetic Survey ................................................................................. 90

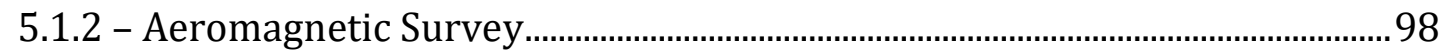

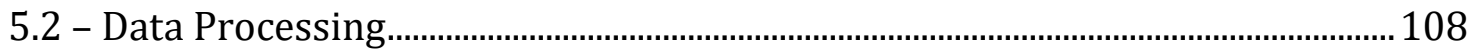

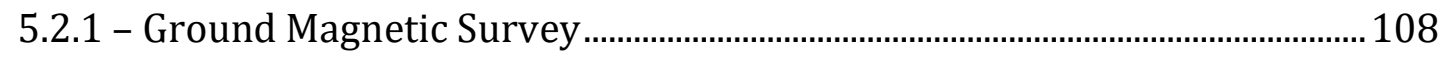

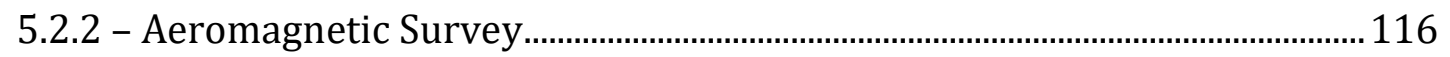

5.2.3 - Ground Magnetic and Aeromagnetic Survey Comparison........................... 124

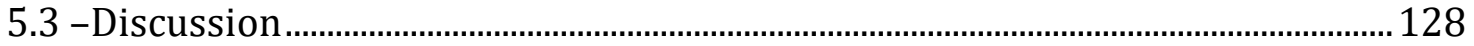

6 - Summary and Concluding Remarks ...........................................................................130

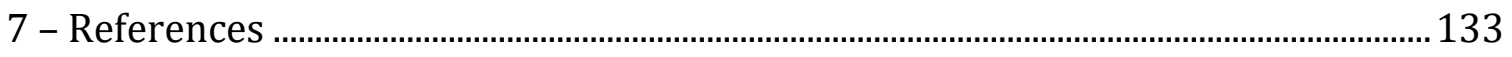

Appendix A - Derivation of gradiometry error propagation ...........................................139

Appendix B - First derivative of the total magnetic field .................................................. 141

Appendix C - Minimum detection capability surface plots ................................................142 


\section{List of Tables}

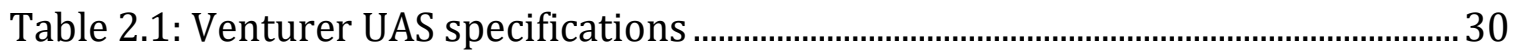

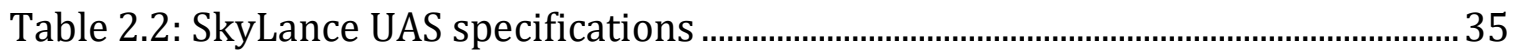

Table 3.1: Specification of the Cessna Grand Caravan ...........................................................40

Table 3.2: Flight speed and spatial sampling intervals for each survey system .........47

Table 5.1: Daily notes for ground magnetic survey ….......................................................96

Table 5.2: Mean flight altitude and attitudes for each SkyLance UAS flight............... 103 


\section{List of Figures}

Figure 2.1: Aeromagnetic anomaly map of North America....................................................

Figure 2.2: Magnetic anomaly map of the Elk Creek deposit in Nebraska, USA..............8

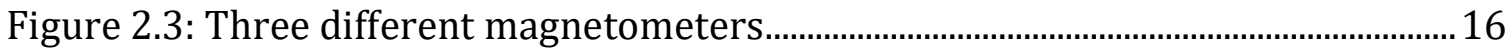

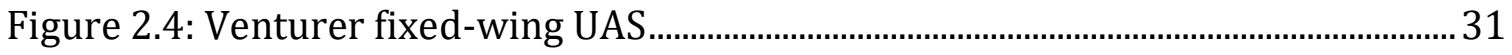

Figure 2.5: SkyLance rotary-wing UAS....................................................................................36

Figure 3.1: A scale comparison of three aeromagnetic systems considered ..................39

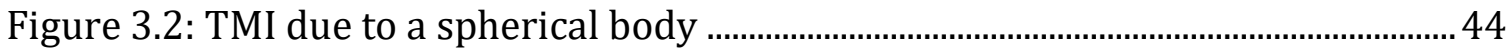

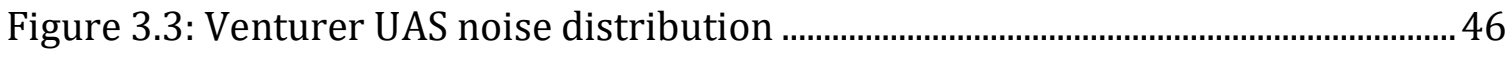

Figure 3.4: $\chi^{2}$ GOF test comparison .......................................................................................50

Figure 3.5: Spherical body detection success rates .............................................................51

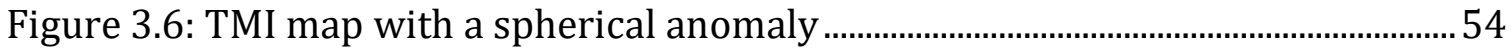

Figure 3.7: Gradiometry error as a function of magnetometer separation .....................55

Figure 3.8: Magnetic anomaly signals caused by pairs of magnetic bodies.....................58

Figure 3.9: Surface plot of the minimum detection limits for each survey system.....64

Figure 3.10: Minimum detection limits for a magnetic body ……………………….........65

Figure 3.11: Transverse magnetic gradient maps ……………………………………...67

Figure 3.12: Resolution limit plots between two magnetic bodies ....................................69

Figure 3.13: Resolution limit for the three survey systems ……………………………... 70

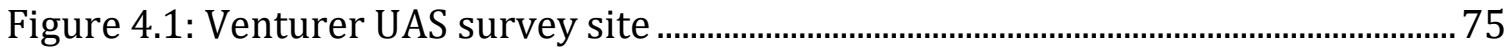

Figure 4.2: Claresholm, $\mathrm{AB}$ regional residual magnetic field variation ............................76

Figure 4.3: Venturer UAS flight path of the experimental survey .......................................77

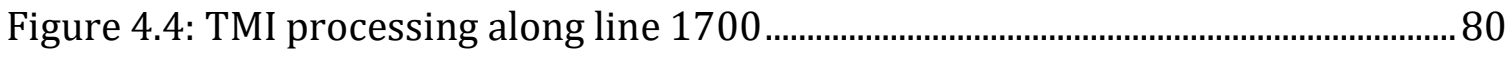

Figure 4.5: Venturer UAS fourth difference noise envelope ...............................................82

Figure 4.6: Venturer UAS TMI map of experimental survey area ......................................85

Figure 5.1: SkyLance UAS survey area with regional geology …………………………...8 
Figure 5.2: Aeromagnetic survey of Nash Creek, NB .........................................................89

Figure 5.3: Ground magnetic survey terrain and bush...................................................... 92

Figure 5.4: Nash Creek, NB survey area and survey lines ....................................................93

Figure 5.5: Elevation profile of line 1550...............................................................................94

Figure 5.6: Preliminary ground magnetic survey map ........................................................97

Figure 5.7: The SkyLance UAS survey team..............................................................................99

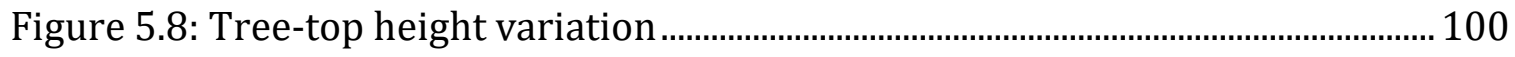

Figure 5.9: SkyLance UAS flight line 1450 ……………………………………………….. 104

Figure 5.10: SkyLance UAS flight altitude variation ......................................................... 105

Figure 5.11: Map view of raw TMI data from the SkyLance UAS flights ....................... 106

Figure 5.12: Profile of raw TMI data from the SkyLance UAS ........................................... 107

Figure 5.13: Diurnal TMI variation for the three ground magnetic survey days ..... 111

Figure 5.14: Processed ground magnetic survey walking path ...................................... 112

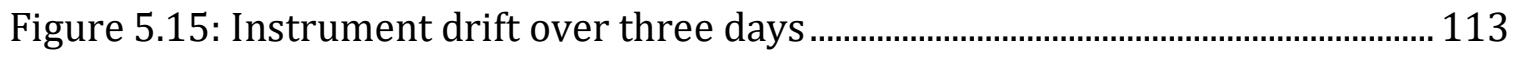

Figure 5.16: Interpolated TMI ground magnetic survey map .........................................114

Figure 5.17: Profile view of TMI along line 1450 ………………………………………... 115

Figure 5.18: SkyLance UAS magnetic data during system power-up............................ 118

Figure 5.19: SkyLance UAS fourth difference noise envelope ........................................ 119

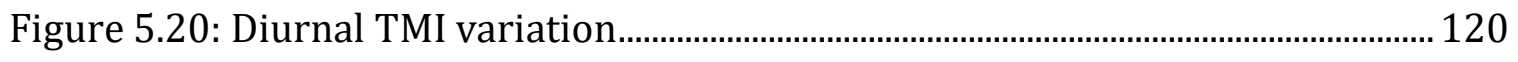

Figure 5.21: TMI variation related to pitch and roll ........................................................ 121

Figure 5.22: TMI variation related to pitch - zoomed in .................................................. 122

Figure 5.23: Profile view of processed SkyLance UAS aeromagnetic survey ............. 123

Figure 5.24: Upward continuation TMI maps of the ground magnetic survey .......... 125

Figure 5.25: TMI map of SkyLance UAS compared with upward continuation.......... 126

Figure 5.26: Profile TMI of SkyLance UAS compared with upward continuation....127

Figure C.1: Surface plots of minimum detection limits for 100m flight altitude....... 142

Figure C.2: Surface plots of minimum detection limits for 50m flight altitude ......... 143 
Figure C.3: Surface plots of minimum detection limits for $2 \mathrm{~m}$ flight altitude ............ 144 


\section{1 - Introduction}

Unmanned aircraft systems (UASs) have been in continuous development at a rapid pace over the past decade (Pajares, 2015). With the improvements in the UASs themselves as well as in the sensors installed onboard, UASs are beginning to replace traditional manned aircraft or satellites in remote sensing projects. Most of these projects involve different forms of ground imagery (visible spectrum, infrared, multispectral, and hyperspectral), yet some UASs are being developed to perform geophysical surveys; more specifically aeromagnetic surveys.

UASs are called several names: unmanned air/aerial vehicle (UAV); remotely piloted aircraft system (RPAS); model aircraft; remote control aircraft; and drone. In most cases these names are synonyms and are used interchangeably. Within Canada UASs have become popular for both recreational and commercial purposes. A UAS is considered recreational if it is not being used for providing a service to someone else and its weight is under 35kg (Transport Canada, 2015). Typically, recreationalists describe their UASs as a model aircraft. The terms UAS, UAV, RPAS, and drone tend to be associated with more complex commercial operations in which operators are performing a service for financial return. These commercial UASs will typically be accompanied by a remote control station along with a communication system (Pajares, 2015). An unmanned aircraft system, as opposed to an unmanned air vehicle, is considered a full system that includes not only the aircraft but the on-board sensors, a remote control station, and all other ground support elements (i.e. communication links, and take-off, landing and recovery systems). 
UASs are automated vehicles that are controlled by the operator through the input of waypoints for the on-board autopilot to follow. Currently, these systems are, at best, semi-autonomous. The systems are capable of performing simple tasks without operator intervention (in-flight manoeuvers, take-off and landing, as well as simple collision avoidance) via the autopilot; however, they cannot perform more advanced tasks without operator intervention. An autonomous vehicle is a system that is self-governing and capable of making its own decisions (such as much more advanced collision avoidance).

An emerging application for UASs is geophysical surveying. This is a branch of the applied geophysics field, which includes methods for "making and interpreting measurements of physical properties of the Earth to determine sub-surface conditions, usually with an economic objective, e.g. discovery of fuel or mineral deposits" (Reynolds, 2011). Geophysical survey methods include, but are not limited to: gravity; seismic reflection and refraction; electrical resistivity; induced polarisation; ground penetrating radar; radiometrics; electromagnetics; and magnetics. The focus for this thesis is the magnetic survey method, with the use of UASs.

\section{1 - Research Objectives}

The research presented in this thesis is focussed on aeromagnetic surveying using UASs, specifically the Venturer fixed-wing UAS, and the SkyLance rotary-wing UAS. These systems have been designed to perform high-resolution aeromagnetic 
surveys for mineral exploration, to identify metallic ore bodies that have a magnetic signature.

There are three main objectives of this research project:

1) To determine the theoretical detection capabilities of two UASs flying at different altitudes, $50 \mathrm{~m}$ and $2 \mathrm{~m}$ above ground level (AGL), and to compare their performance to that of a traditional fixed-wing system flying at $100 \mathrm{~m}$ AGL. This theoretical analysis is shown in Chapter 3.

2) To demonstrate the aeromagnetic survey capabilities of the Venturer fixed-wing UAS. Chapter 4 presents the data acquired by the Venturer during a survey flown near the town of Claresholm, AB.

3) To demonstrate the aeromagnetic survey capabilities of the SkyLance rotary-wing UAS by comparing it to a ground magnetic survey. These surveys were performed in Nash Creek, NB, and are discussed in detail in Chapter 5. 


\section{2 - Background Information}

The following two sections provide an overview of the background theory for aeromagnetic surveying (Section 2.1), as well as further details into UASs (Section 2.2). In Section 2.1 an overview of the magnetic theory is provided followed by a description of how aeromagnetic surveying is performed, and what type of magnetic sensors (magnetometers) are used. Section 2.2 discusses some current UAS applications and provides a detailed description of the two UASs flown as part of this research project; the Venturer fixed-wing UAS and the SkyLance rotary-wing UAS.

\section{1 - Magnetic Methods}

Due to fluid motion within the Earth's outer iron core, a magnetic field is generated and maintained; this is known as a geodynamo. The metallic outer core is an electrically conductive fluid that rotates and convects. It is in a continual state of convection due to both chemical differentiation and radioactive heating (Ranalli, 1995). This allows for a process that is essentially a naturally occurring electrical generator where the kinetic energy within the outer core is converted to electrical and magnetic energy. The fluid motions within the outer core induce electric currents within itself. The currents, in turn, generate their own magnetic fields. This process is self-sustaining due to internal feedback within the outer core. The magnetic field of the Earth will interact with magnetically susceptible bodies on and below the Earth's surface. 
Magnetic surveying is performed to investigate the subsurface to improve the understanding of the regional or local geological setting and history; or to identify potential economic resources (mineral deposits). Magnetic anomalies are variations (high or low) of the Earth's local magnetic field resulting from the magnetic properties of underlying rocks and minerals (Keary, et al., 2002). Most minerals that compose a rock are non-magnetic; however, there are some minerals that have sufficient magnetic susceptibility that they can cause an increase or decrease of the local magnetic field on or near the surface of the Earth. The Earth's magnetic field fluctuates globally depending on location with typical magnetic field measurements at high latitudes ranging between 50,000nT and 60,000nT (Keary, et al., 2002).

Aeromagnetic surveying is performed at two different levels: regional and local. Through regional, large-size surveying, it is possible to identify and follow major geological trends. Most of North America has been mapped magnetically, as seen in Figure 2.1 (United States Geological Survey, 2015). In Figure 2.1 it is possible to follow geological units across North America: the Rocky Mountain belt on the Western coast of North America and the Grenville Orogeny mostly in Ontario and Quebec (Tollo, et al., 2004). More local higher resolution surveys are performed for mineral exploration purposes. These surveys are performed in the hopes of finding a geological structure that has or is associated to an ore body of economic value; such as precious metals or rare earth elements. An example of a magnetic map that contains a magnetic anomaly is shown in Figure 2.2. The data in Figure 2.2, of Elk Creek, Nebraska, USA, have been used to identify potential economic mineral deposits 
containing carbonatite hosting niobium and other rare earth elements (Drenth, 2014). 


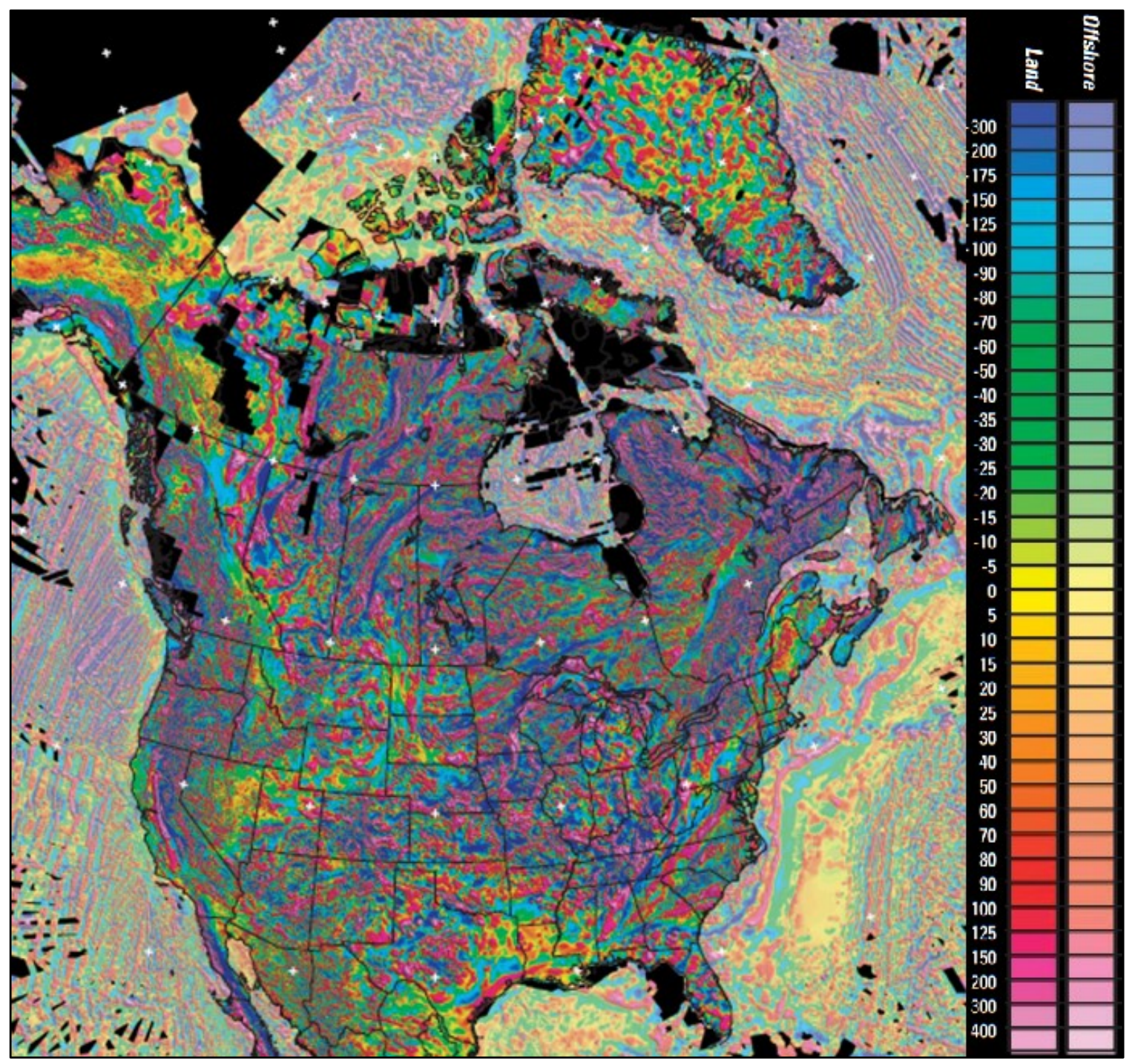

Figure 2.1: Residual aeromagnetic anomaly map of North America. Colour bar units are in nano-teslas (nT) (United States Geological Survey, 2015). 


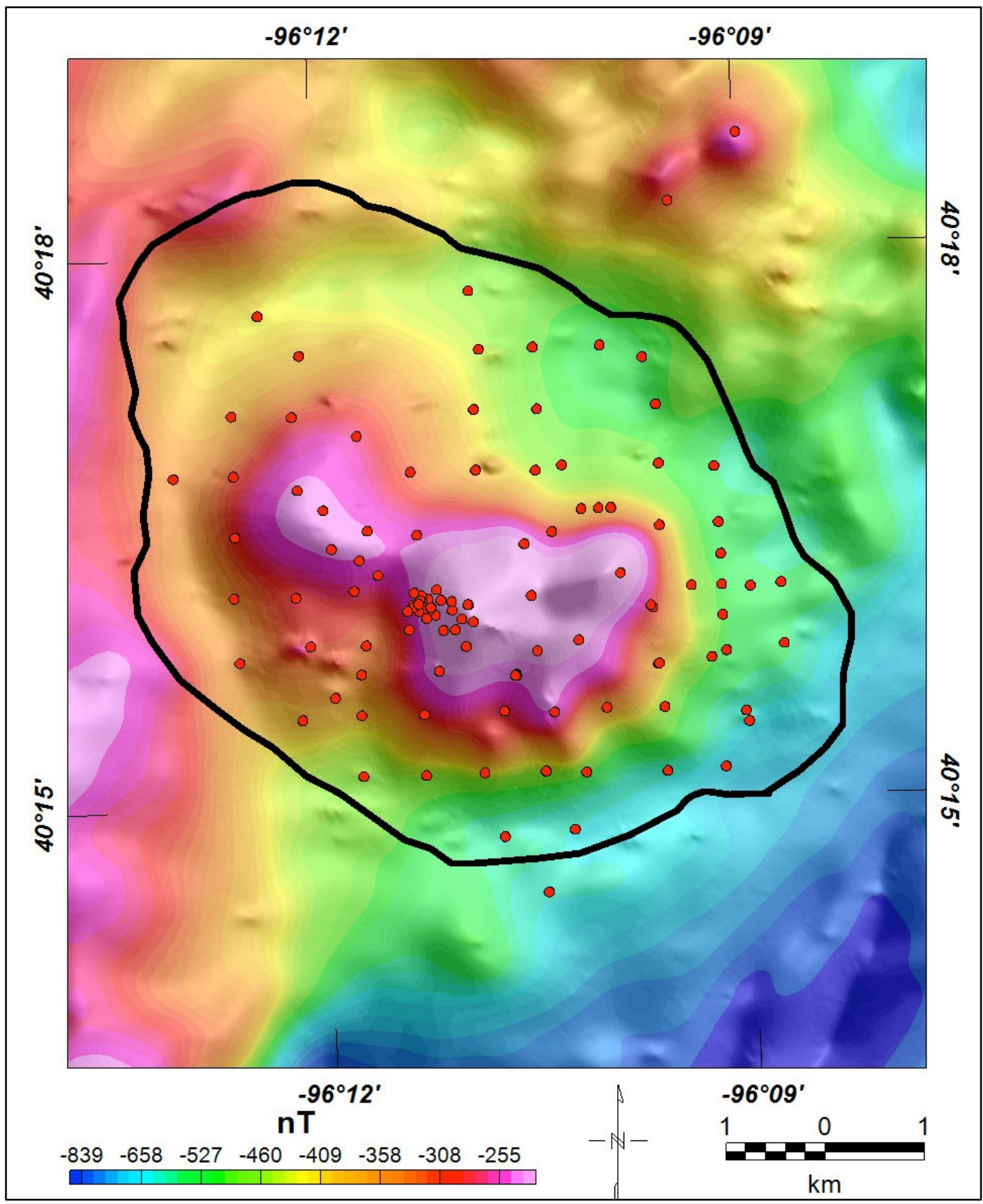

Figure 2.2: Residual magnetic anomaly map of the Elk Creek deposit in Nebraska, USA. Red dots are borehole locations (Drenth, 2014). 


\subsection{1 - Magnetic Induction Principles}

The magnetic induction principles are important for understanding how a magnetic anomaly, due to a mineral or rock body within the Earth's subsurface, will affect the Earth's magnetic field. The following will explain the induction principles, which are important for the theoretical analyses performed in Chapter 3.

The magnetic effect from electrical currents and magnetic materials can be obtained from Coulomb's law for magnetic poles (Telford, et al., 1976),

$$
\boldsymbol{F}=\frac{m_{1} m_{2}}{4 \pi \mu r^{2}} \boldsymbol{r}
$$

where $\boldsymbol{F}$ is the force vector in Newtons which is acting on pole $m_{2}$ from pole $m_{1}$. Variable $r$ is the distance between the two poles and $\boldsymbol{r}$ is a unit vector directed from $m_{1}$ to $m_{2}$. The permeability of the medium surrounding the magnetic poles $\left(m_{1}\right.$ and $m_{2}$ ) is $\mu$.

A quantity that is more widely used in practice is the magnetic field strength from a magnetic pole of strength $m$.

$$
\boldsymbol{H}=\frac{\boldsymbol{F}}{m_{2}}=\frac{m}{4 \pi \mu r^{2}} \boldsymbol{r}
$$

The field strength $(\boldsymbol{H})$ can be produced as a function of the magnetized material or also by the current flowing within material (i.e. a wire).

Magnetic poles always exist in pairs, so the fundamental body is known as a magnetic dipole where two poles of strength $+m$ and $-m$ are separated by a distance 
$l$. This defines the magnetic moment $(\boldsymbol{M})$ a vector in the direction of $\boldsymbol{r}$ extending from the negative pole towards the positive pole; a property that determines the force that a magnet can exert on a body.

$$
\boldsymbol{M}=m l \boldsymbol{r}=M \boldsymbol{r}
$$

If a magnetic body is placed in an external magnetic field, it can become magnetized by induction. The intensity $(I)$ of magnetization is proportional to the field strength and direction.

$$
\boldsymbol{I}=\boldsymbol{M} / \mathrm{v}=\boldsymbol{I} \boldsymbol{r}
$$

The degree to which a body is magnetized is known as the magnetic susceptibility $(k)$. Magnetic susceptibility is a fundamental rock property in magnetic surveying. The magnetic response from rocks or minerals is determined by the amount of magnetic material in them or how strong $k$ is compared to surrounding rocks and minerals.

$$
k=I / H \text { or } \boldsymbol{I}=k \boldsymbol{H}
$$

A magnetic body that is placed within an external field $(\boldsymbol{H})$ will produce its own field $\left(\boldsymbol{H}^{\prime}\right)$. This magnetic field will increase the total magnetic field $(\boldsymbol{B})$ by magnetic induction.

$$
\boldsymbol{B}=\boldsymbol{H}+\boldsymbol{H}^{\prime}=\boldsymbol{H}+4 \pi \boldsymbol{I}=(1+4 \pi k) \boldsymbol{H}=\mu \mathbf{H}
$$

From equation 2.6, we see that the relationship between magnetic susceptibility $(k)$ and magnetic permeability $(\mu)$ is shown in equation 2.7 


$$
\mu=(1+4 \pi k)
$$

\subsection{2 - Total Magnetic Intensity}

The magnetic signals from rocks or minerals that are superimposed onto the Earth's magnetic field will create magnetic anomalies (Teskey, et al., 1991). The Earth's magnetic field itself, because it is a vector quantity, varies in both amplitude as well as direction depending on the location on the Earth. Furthermore, the direction of the induced magnetic field from the rock or mineral in the Earth's subsurface might have a magnetic field vector which could be pointing in a different direction than the Earth's magnetic field. Instead of recording the vector components of the Earth's magnetic field, many magnetic surveys will record the total magnetic intensity (TMI); that is, the amplitude of the magnetic field vector. This approach simplifies the processing of the magnetic data and still allows for the identification of local magnetic anomalies associated to TMI variations.

\subsection{3 - Gradiometry}

Magnetic gradiometry is a survey technique that identifies locations where there is a large change in TMI over short distances (Hood \& Teskey, 1989). The magnetic gradient is defined as the difference between two magnetic measurements

$\left(B_{1}\right.$ and $\left.B_{2}\right)$ divided by the separation distance between the two measurements $\left(x_{1}\right.$ and $x_{2}$ ). 


$$
\frac{\Delta B}{\Delta x}=\frac{B_{1}-B_{2}}{x_{1}-x_{2}}
$$

Typically multiple magnetometers located at a fixed separation and orientation are used to make gradiometric measurements. These magnetometers can be spaced horizontally or vertically to measure either the horizontal or the vertical gradient of the magnetic field. Transverse gradiometry is the horizontal gradient measured perpendicular to the survey line. It is also possible to measure the gradient using two successive measurements from the same magnetometer that were recorded at two different positions, this is known as in-line gradiometry.

The use of gradiometry is mainly employed to identify shallow magnetic features (Keary, et al., 2002), where the gradient(s) will assist in resolving complex anomalies into their individual components. An advantage of gradiometry is that it allows for the automatic removal of temporal and regional magnetic variations, like diurnal effects.

\subsection{4 - Magnetometers}

Civilization has been using magnetic devices for centuries. The first known device that responded to the Earth's magnetic field was used by Chinese diviners in the first century $\mathrm{AD}$, while compass needles have been used in Asia since approximately the year 1000 for navigation (Reynolds, 2011). The methods and devices geophysicists now use to measure the Earth's magnetic field intensity and 
vector components were only introduced in the early 1900's (Keary, et al., 2002); these devices are known as magnetometers.

Currently there are a few different types of magnetometers with different advantages and limitations when compared to one another. These include the fluxgate magnetometer; the proton precession magnetometer and the optically pumped magnetometer (Keary, et al., 2002).

The fluxgate magnetometer (Figure 2.3 - top) has been in use since the 1940s. It uses a set of primary and secondary coils, and while in an external magnetic field induced voltages in each coil become out of phase. The output of these coils is proportional to the external magnetic field component being investigated. Thus, most fluxgates contain sets of coils that are orientated in each of the three cardinal axes, allowing for the measurement of the magnetic field vector components. However, the fluxgate magnetometer is only accurate to about $\pm 1 \mathrm{nT}$; this is much less precise than other magnetometers. Typically fluxgates are used for airborne flight orientation instead of magnetic field surveying. Both of the UASs used in this project, the Venturer UAS (Section 2.2.1) and the SkyLance UAS (Section 2.2.2) make use of the fluxgate magnetometer for this purpose.

The proton precession magnetometer (Figure 2.3 - middle) is the most commonly used magnetometer (Keary, et al., 2002). The sensor consists of a bottle containing a proton-rich liquid, such as water or kerosene, which is wrapped by a coil to which the measuring or recording device is connected (Reynolds, 2011). A current is applied through the coil, generating a magnetic field about 50 to 100 times stronger 
than the Earth's magnetic field. This causes protons to align to the new magnetic field. Once the current in the coil is switched off, the polarized protons will then rapidly return to their original alignment to the external magnetic field by spiralling or precessing. This precession has a frequency that can be related to the external field (i.e. Earth's magnetic field). This technique allows for the measurement of the TMI to a precision of approximately $\pm 0.1 \mathrm{nT}$. However, due to the time required to turn the current on and off these devices are not continuously recording; typically 1 to 3 seconds are required between recordings (Keary, et al., 2002).

The latest generation of proton precession magnetometers has taken advantage of a phenomenon known as the Overhauser Effect. To perform this, a liquid is added to the proton-rich fluid that contains free, unpaired orbit electrons. This allows for the protons to become polarized at lower power levels $(75 \%$ decrease in power consumption), produces a signal approximately 100 times stronger than that of the electron-deficient fluid, and reduces the noise levels. This style of magnetometer is primarily used for ground magnetic surveys, but it is also usable in airborne (or sea) surveys as well (Reynolds, 2011). An Overhauser magnetometer was used for ground magnetic surveying in Nash Creek, New Brunswick as part of this project (see Chapter 5).

A third type of magnetometer is the optically pumped magnetometer (Figure 2.3 - bottom) which is a TMI sensor that can achieve much higher precision than other magnetic sensors (up to $\pm 0.01 \mathrm{nT}$ ). Within the sensor an evaporated alkali metal such as cesium (in the case of the cesium vapour magnetometer), potassium, or rubidium 
is contained within a glass cell. This vapour is energized by light of a specific wavelength so that the atoms will have their electrons, that exist in its valence energy levels (A and B), excited to a higher unstable energy level (C). These high valence electrons will then decay back to their original energy levels. Repeating this process is known as optical pumping and it will cause the first energy level (A) to become fully populated, but the second energy level (B) will be underpopulated. The energy difference between valence levels $A$ and $B$ is proportional to the external magnetic field being measured. This type of magnetometer is primarily used in airborne magnetic systems, since its precision capabilities are primarily desirable for gradiometry measurements (Keary, et al., 2002). Both the Venturer (Section 2.2.1 and Chapter 4) and SkyLance UASs (Section 2.2.2 and Chapter 5) used this style of magnetometer for the aeromagnetic surveys during this project. 

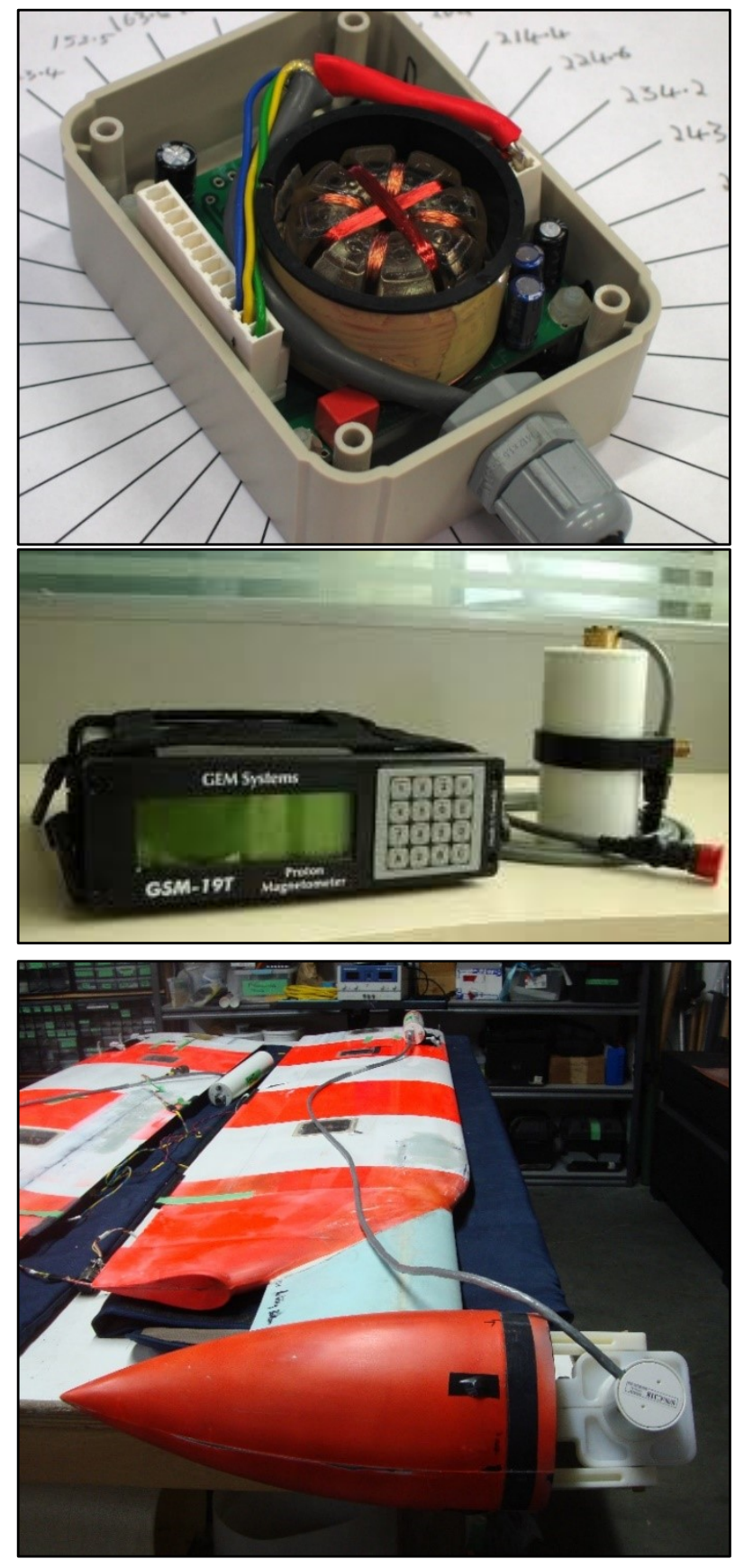

Figure 2.3: Three different magnetometers. Top - Fluxgate magnetometer which can be smaller than about $8 \mathrm{~cm}$ in length and $4 \mathrm{~cm}$ wide, and as light at $150 \mathrm{~g}$. Photo source: Wikipedia (2015); Middle - Overhauser proton-precession magnetometer developed by GEM systems. The console (left) is about $24 \mathrm{~cm}$ long and $22 \mathrm{~cm}$ wide and the sensor is connected to a $1.7 \mathrm{~m}$ long pole providing a total weight of about $4.3 \mathrm{~kg}$. Photo source: Trade India (2015); Bottom - Cesium vapour magnetometer mounted within the Venturer UAS wingtip pod. This Geometrics magnetometer sensor is about $6 \mathrm{~cm}$ in diameter and $16 \mathrm{~cm}$ long with a weight of about $340 \mathrm{~g}$ without the connecting cable. 


\subsection{5 - Data Processing}

Raw magnetic survey data can contain undesirable magnetic effects for mineral exploration purposes. These noise effects can include: random effects from the magnetometers themselves and nearby electrical components; effects from changing attitude and position of the survey system; vibrations of the system; aircraft heading effects; and magnetic field variation due to diurnal effects of the sun and the moon. The effect from the sun is approximately 30 times stronger than the moon (Keary, et al., 2002; Matsushita and Maeda, 1965). Before performing geological interpretation, various corrections to the magnetic survey data must first be applied.

Aeromagnetic surveys typically perform in-flight real-time attitude compensation, removing effects due to the changing attitude of the aircraft, before any data analysis is performed. This is typically done by on-board hardware that is fed the aeromagnetic and attitude data prior to recording it. Effects removed are variations in magnetic field measurements due to: remanent magnetism (permanent magnets on-board); induced magnetization within the aircraft that is generated by the Earth's magnetic field; and eddy currents generated by electrical conductors that are also moving through the Earth's magnetic field (Leach, 1979). The attitude of the aircraft is described in terms of an 18 coefficient tensor which is solved by flying a figure of merit (FOM) pattern (Mandea \& Korte, 2011) then solved through the use of linear least squares regression (Leach, 1979), neural networks (Williams, 1993), or other methods. This is done to remove effects in aeromagnetic data due to a change in the aircrafts attitude in the sky. This FOM pattern is usually a "figure 8" pattern so 
that directional effects in all directions are captured in order to calibrate the processing algorithms. It is performed at very high altitudes to remove influences from geological and cultural sources. At the current stage of development for UASs, such as for the Venturer and SkyLance, there is no attitude compensation hardware available and the high-altitude figure of merit cannot be performed because UASs are not designed or allowed to fly at such heights. The compensation of UAS magnetic data is an open research question within the community, and is not addressed in this research project. The implementation of an attitude compensation system for UASs could decrease the noise levels in the aeromagnetic data enough such that they could be within industry standards (see Section 2.1.5.1).

Further details on noise analysis performed on aeromagnetic data (the fourth difference); corrections applied to magnetic data to remove diurnal and heading effects (the diurnal correction and tie-line levelling); as well as post-processing techniques to bring various survey datasets to the same elevation (upward continuation) are presented in the following sub-sections (2.1.5.1 - 2.5.1.4).

\subsubsection{1 - Fourth Difference}

The noise in aeromagnetic data can come from not only the recording device but can also be partially caused by the survey aircraft itself and can include either static or dynamic effects (Teskey, et al., 1991). Static effects are due to permanent or induced magnetic fields from components on-board the aircraft. Induced currents within the aircraft frame or other metallic (ferrous) components can cause a change 
in the intensity of the magnetic readings which depends on the aircraft's heading (direction the aircraft is pointing with respect to magnetic north). Dynamic effects are created from the motions of on-board components and the airframe itself during flight; such as the aircraft's wings oscillating up and down.

Both static and dynamic effects can produce either discontinuous or continuous noise. Discontinuous noise appears as an isolated spike or set of closelyspaced spikes on aeromagnetic data. Causes for this type of noise can often be related to the crew's actions such as radio transmissions or switching DC current output. External causes could include lightning strikes or cultural sources (e.g. train, power lines). Continuous noise comes from the motions of the aircraft, such as the oscillation of wings (flexing) while in flight through turbulent weather, which produce a highfrequency unwanted signal. Further effects could be observed through manoeuvers performed to keep the aircraft on a flight line or turning to the next flight line.

The fourth difference calculation is a method for determining the levels of noise in the aeromagnetic data used by the survey operators to ensure that these noise effects are within an acceptable level.

$$
4 \text { th Difference }=-\frac{X_{-2}-4 X_{-1}+6 X_{0}-4 X_{1}+X_{2}}{16}
$$

The fourth difference is calculated at the magnetic value $X_{0}$, at time $t_{o}$, and considers the two previous $\left(X_{-2}\right.$, and $\left.X_{-1}\right)$ as well as the following two $\left(X_{1}\right.$, and $\left.X_{2}\right)$ recorded values in time. This empirical function (Eq. 2.9) allows for monitoring of the aeromagnetic survey systems noise levels while in flight or in post-processing. For 
the data to be considered acceptable for commercial surveys, the fourth difference noise levels must be within an envelope of $\pm 0.05 \mathrm{nT}$ ( $0.1 \mathrm{nT}$ peak-to-peak) (Coyle, et al., 2014). If there are any issues with the data quality, the contractor must resolve these issues before the commercial survey can continue.

\subsubsection{2 - Diurnal Correction}

Magnetic effects due to external origins that cause daily variations in the Earth's magnetic field are known as diurnal variations (Keary, et al., 2002). These variations are typically smooth and regular with an amplitude range of approximately 20 to $80 \mathrm{nT}$. They result from the magnetic field induced by the motion of charged particles from the solar wind interacting with the Earth's ionosphere.

Diurnal variations can be removed via several methods (Keary, et al., 2002); however, the preferred, and most effective, technique is to use a stationary magnetic sensor on the ground as a magnetic base station (Teskey, et al., 1991). This base station will operate over the full aeromagnetic survey time period, where it will continuously record the Earth's magnetic field. According to the time of observation, the magnetic base station readings are removed from the aeromagnetic survey data. This allows for the removal of temporal variations in the same data as well. 


\subsubsection{3 - Tie-line Levelling}

After attitude (real-time attitude compensation, if applied) and diurnal corrections are applied to aeromagnetic data, there still may be residual errors which are usually small but can still provide undesirable artifacts between survey lines (Minty, 1991). Data can suffer from spurious cross-line variations or levelling errors (Saul \& Pearson, 1998). These effects can be due to navigational inaccuracies of the system such as: different flight altitudes between each line; the magnetic sensor being located some distance away from the on-board GPS causing a parallax error; effects due to different aircraft attitudes (roll, pitch, and yaw); and magnetometer instrument drift (Luyendyk, 1997). There are multiple methods for correcting the errors mentioned above, including: loop closure methods; gridding and contouring; micro-levelling; and polynomial levelling (Saul \& Pearson, 1998). Levelling methods require data from cross-over lines (tie-lines) perpendicular to the survey lines of an aeromagnetic survey. The locations in which the survey lines and the tie-lines intersect are known as cross-over points.

The technique used in this research project is polynomial levelling. Polynomial levelling fits a polynomial function to the cross-over points along a survey line by using the method of least squares fitting (Luyendyk, 1997). The fitted polynomial data is then subtracted from the original data, which minimizes the intersection errors between the survey lines and tie-lines without requiring that the data be exactly matched (since data may contain noise). 


\subsubsection{4 - Upward Continuation}

Magnetic surveys can be performed at various altitudes AGL. For sound comparisons between different datasets the magnetic data needs to be at a consistent altitude. Upward continuation is a processing technique that allows for different magnetic surveys to be compared or merged even though they were flown at different altitudes. It brings each survey up to a consistent altitude. Occasionally upward continuation is also applied to enhance anomalies caused by deeper, larger sources and attenuate anomalies caused by shallow, small sources (Blakely, 1996). Upward continuation transforms the potential field measured on one surface to the field that would be measured on another surface farther away from the source (Wang, 2006).

Upward continuation is typically performed by using the Fast Fourier transform (FFT) technique. The recorded data is Fourier transformed into the frequency spectrum, then multiplied by an exponential, upward continuation filter (Eq. 2.10). The data is then transformed back into the time domain by the inverse FFT.

$$
\text { exponential filter }=e^{-\Delta z|k|}
$$

The variable $k$ is the wavenumber, and $\Delta z$ is the positive change in altitude (Blakely, 1996). 


\section{2 - Unmanned Aircraft Survey Systems}

Remote sensing, a technique for capturing information from a distance, is traditionally performed by manned aircrafts or satellites (Pajares, 2015). Over the past decade there has been rapid development of UASs as well as of the remote sensing equipment installed onboard. UASs are becoming a desirable alternative or a complementary approach for remote sensing since they allow for high versatility and flexibility as a platform in comparison to traditional airborne and satellite systems. Operations with manned systems also pose high risks to humans (such as low altitude flying and operator fatigue) which are mitigated with the use of UASs. Furthermore, UASs are also capable of flying at very low altitudes (some as low as 2m AGL) and at much slower flight speeds, giving them the ability to acquire higher resolution (spatially and temporally) data.

UASs are being developed for many different applications (Pajares, 2015). Military applications: targeted drone attacks; border patrolling; and surveillance. Imaging applications for UASs include: video cameras in the visual spectrum; thermal infrared video sensors, which detect a different emitted wavelength of light that is related to the temperature of the emitting body; Lidar systems used for surveillance and mapping, which measure the reflection travel time for light emitted by a laser; and multispectral and hyperspectral sensors, which build coherent images through line scanning of various specific light frequencies. Non-electromagnetic (light) applications include: chemical sensors, which measure amounts of water vapour $\left(\mathrm{H}_{2} \mathrm{O}\right)$, carbon dioxide $\left(\mathrm{CO}_{2}\right)$, methane $\left(\mathrm{CH}_{4}\right)$, etc.; sonar systems used to measure 
distances and perform surface topographical mapping; and atmospheric instruments used for detecting particles $(>10 \mathrm{~nm}$ in size) in the atmosphere, measuring concentrations of particles, determining temperature and relative humidity, estimating cloud droplet size, and the amount of solar radiation. Radiation detection UASs have also been developed to measure gamma rays due to nuclear and radioactive accidents, incidents, and sources on the ground or in the atmosphere. Lastly, magnetometers have been mounted on UASs to generate detailed magnetic maps and to identify magnetic sources in the Earth's subsurface (Pajares, 2015).

Magnetic surveying with UASs have been gaining momentum in the mineral exploration industry. There is a demand in the industry for high-resolution, smallsize surveys to be flown at lower altitudes than traditional methods (airborne surveying with fixed-wing and rotary-wing manned aircraft, and ground magnetic surveying) (Barnard, 2008). UASs represent an opportunity to conduct such detailed surveys, in particular in remote areas such as arctic and offshore regions. They require fewer personnel than their traditional counterparts without the risk for pilots who would be flying at low-altitudes (Kroll, 2013).

Currently, there are various UASs under development for aeromagnetic surveying. Primarily these systems are fixed-wing UASs such as the GeoRanger (Fugro/CGG) - a modified fixed-wing UAS for geophysical applications (now retired), which was flown for several missions (Anderson \& Pita, 2005); and the GeoSurv II a UAS specifically designed for aeromagnetic surveying and built as a joint effort between Sander Geophysics and Carleton University (Caron et al., 2014; Caron et al., 
2011; Samson et al., 2010). More recently Abitibi Geophysics and GEM Systems partnered to introduce the AeroVision fixed-wing UAS on the market (Dion-Ortega, 2015). The Surveyor UAV is under development by Pioneer Exploration Consultants Ltd. and is one of the few small rotary-wing UAS (Burns, 2015). This thesis focuses on two UASs under development by Stratus Aeronautics Inc. specifically for aeromagnetic surveying: the Venturer fixed-wing UAS and the SkyLance rotary-wing UAS.

\subsection{1 - Venturer UAS}

The specifications of the Venturer UAS (Figure 2.4) are listed in Table 2.1. The airframe of the UAS is constructed primarily from carbon fiber and fiber glass composite materials, which are non-magnetic. Carbon fiber is used for the fuselage and the main structure of the wing. Fiberglass is used for the fin and magnetometer sensor pods. The fiber glass composite ensures no electromagnetic interference with magnetometer instrumentation. The UAS uses a two-stroke gasoline powered engine for propulsion and is coupled to an electrical generator to power the on-board avionics and payload electronics. Auxiliary backup power is provided by lithium batteries which are kept charged by the generator during flight. This distribution of power from the generator to the various on-board systems (system monitors, avionics, payload, and batteries) is controlled by a power management system. The avionics components consist of an autopilot, differential GPS, and servos. The payload consists of an inertial measurement unit (IMU); a fluxgate magnetometer; and two 
cesium vapour magnetometers. The two cesium magnetometers are mounted in pods at each wing tip (as shown in Figure 2.3 - bottom) and have a sampling frequency of 10Hz. A differential real-time kinematic (RTK) GPS unit is used to provide a positioning accuracy of approximately $10 \mathrm{~cm}(2 \mathrm{~cm}$ if within the home base range). The Venturer UAS measures above sea level (ASL) altitude with a barometric altimeter connected to a pitot-static tube system. AGL altitude measurements are made with a laser and/or a radar altimeter. It is also possible to use the ASL readings in conjunction with a digital elevation model to determine the AGL.

The Venturer UAS is designed to fly at an optimum airspeed of $95 \mathrm{~km} / \mathrm{h}$. It would be unsafe for the aircraft's integrity to vary the airspeed to achieve a constant ground flight speed. Atmospheric conditions (e.g. wind gusts, wind shear, and crosswinds) can alter the speed of the air over the flight surfaces and consequently affect lift. It is therefore important to fly at speed sufficiently high enough to minimize the effects of these weather phenomena. A recommended minimum airspeed for flight is approximately $80 \mathrm{~km} / \mathrm{h}$, which will vary depending on the amount of fuel on-board and the payload weight. Airspeeds lower than $80 \mathrm{~km} / \mathrm{h}$ increase the risk of stalling the aircraft (the aircraft's stall speed is $50 \mathrm{~km} / \mathrm{h}$ ) and reduce the responsiveness of the flight controls. Obviously flying close to the stall speed is extremely undesirable at low altitudes above ground. These airspeeds were determined for the Venturer UAS using in-house calculations by following the methods laid out in Raymer (2006) and Anderson (2011). 
While in flight, the UAS follows pre-determined waypoints. It is possible to provide waypoints on-the-fly, alter the flight path in real time, re-fly previous survey lines, or return to the airstrip. The on-board autopilot will command the UAS to approach a waypoint as closely as possible without exceeding the specified limits for maximum pitch $\left(20^{\circ}\right)$, and roll $\left(30^{\circ}\right)$ values. These constraints are in place to ensure the safety of the UAS and to prevent any drastic flight path adjustments which can result in high stresses to aircraft structure and possible damage. Once the UAS passes a waypoint, it will continue onto the next waypoint. Good surveying practice, which the Venturer UAS follows, requires that excursions from the flight line be limited to no more than $30 \%$ of the survey line spacing in order to ensure good quality and regular data coverage. The precision to which the aircraft can keep on a flight line is dependent not only upon atmospheric conditions but also the accuracy of the autopilot's "tuning" parameters. These parameters or coefficients are derived from the computer modelling of the aircraft. The operator can command the aircraft to new waypoints or manually take control of the UAS at any time. This manual override may need to be implemented for many reasons which may include deteriorating weather conditions or concerns that the payload may not be acquiring good quality data (for example, in the event of a magnetic storm).

The Venturer UAS has been designed with various features to ensure the safety not only of the aircraft, but also of the environment and other airspace users. These include redundant avionics and power back up systems plus the facility to set up a "geo-fence" which ensures that the aircraft remains within its predefined airspace. Potential problems mitigated for in the design of the Venturer UAS include 
contingencies for abnormalities with the autopilot, communication systems, engine, servos or GPS.

During flight, the UAS communicates with the operator via both satellite and UHF radio frequency (RF) links. The satellite communication link does not allow operator-controlled flight in the same way as it does through the UHF link. It does permit the modification of flight plan waypoints as well as the ability to command the aircraft to perform an immediate return to the airfield or, if necessary, an emergency landing. The UHF radio link supports the full spectrum of capabilities and allows for the operator to manually take control of the aircraft. This is normally only done for take-off and landing; however, in the future it is planned that the entire operation from take-off, mission flight and landing will be done automatically. Depending on local conditions (i.e. weather, terrain and antenna configuration), the UAS can operate at a range from $8 \mathrm{~km}$ to $30 \mathrm{~km}$ using the UHF radio communication link.

The Venturer UAS is highly manoeuvrable and, due to its comparatively low wing loading and small size, capable of flying at low altitude. The lowest altitude which can be flown safely varies depending on several factors including: weather, topography, forest canopy height, and survey design requirements. The maximum altitude depends on the air density, temperature and aircraft weight (including payload and fuel). With the current 120cc twin cylinder two stroke gas engine, it is possible to reach an altitude of approximately $1,500 \mathrm{~m}$. The planned installation of a fuel injected gas engine will increase maximum altitude in excess of $2,400 \mathrm{~m}$. To 
ensure safe aircraft take-offs and landings, a flat and smooth surface (i.e. tarmac or grass field), approximately $150 \mathrm{~m}$ in length and $20 \mathrm{~m}$ in width, is required. 
Table 2.1: Venturer UAS specifications

\begin{tabular}{|c|c|}
\hline Length: & $2.74 \mathrm{~m}$ \\
\hline Wingspan: & $4.95 \mathrm{~m}$ \\
\hline Crew size: & 2 people \\
\hline Mass: & Approx. 55kg (with fuel) \\
\hline $\begin{array}{l}\text { Mass of } \\
\text { Payload: }\end{array}$ & $\begin{array}{l}8.28 \mathrm{~kg} \text { (includes magnetic } \\
\text { sensors, computer, power } \\
\text { supplies, and secondary } \\
\text { sensors) }\end{array}$ \\
\hline Endurance: & $9-10 \mathrm{~h}$ at $100 \mathrm{~km} / \mathrm{h}$ \\
\hline $\begin{array}{l}\text { Maximum } \\
\text { flight speed: }\end{array}$ & 120km/h (air speed) \\
\hline GPS: & Differential GPS \\
\hline GPS Accuracy: & $\begin{array}{l}2 \mathrm{~cm} \text { within system base } \\
\text { station range } \\
10 \mathrm{~cm} \text { outside of system base } \\
\text { station range }\end{array}$ \\
\hline Engine: & $\begin{array}{l}\text { 120cc twin cylinder two } \\
\text { stroke gas engine }\end{array}$ \\
\hline Fuel: & $\begin{array}{l}91 \text { octane automotive ethanol } \\
\text { free fuel with } 2 \text {-stroke oil at a } \\
50: 1 \text { ratio }\end{array}$ \\
\hline Servos: & 9 servos ( 6 used in flight) \\
\hline Sensors: & $\begin{array}{l}\text { Two Geometrics G-823A } \\
\text { cesium vapour magnetometers }\end{array}$ \\
\hline IMU: & Micro-electromechanical IMU \\
\hline $\begin{array}{l}\text { Fluxgate } \\
\text { magnetometer: }\end{array}$ & $\begin{array}{l}\text { Applied Physics model } 539 \\
\text { high speed digital 3-axis } \\
\text { magnetometer }\end{array}$ \\
\hline
\end{tabular}




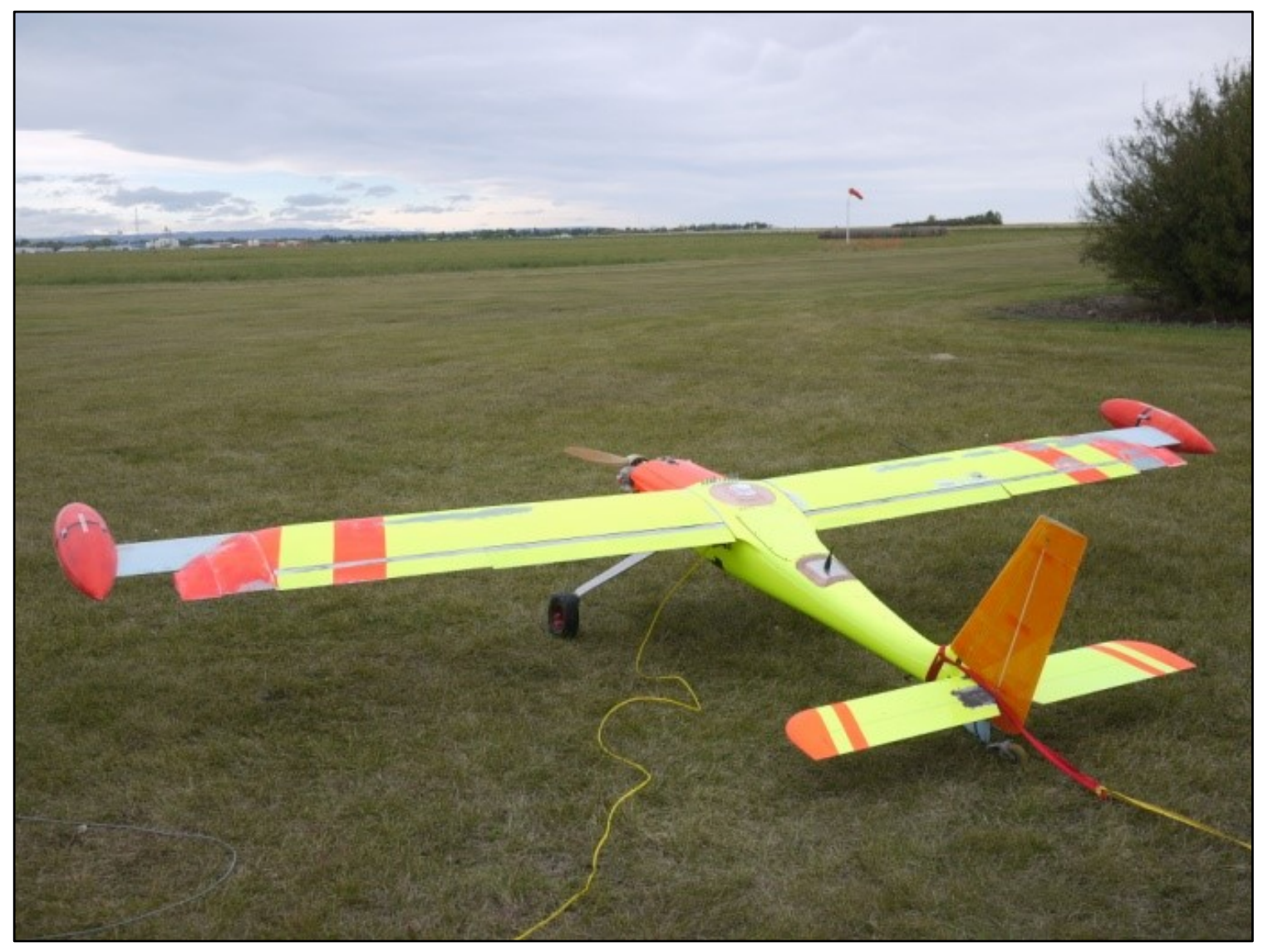

Figure 2.4: Venturer fixed-wing UAS mounted with cesium vapour magnetometers in protective pods on wingtips. The UAS has a wingspan of $4.95 \mathrm{~m}$. 


\subsection{2 - SkyLance UAS}

The specifications of the SkyLance UAS (Figure 2.5) are listed in Table 2.2. The system has been carefully developed with carbon fibre material to eliminate the magnetic interference from the UAS frame on the magnetometers. The UAS has four perpendicular arms where each carry a set of two rotors mounted vertically, allowing the system to utilize a total of eight rotors for propulsion. Four lithium ion batteries power the rotors as well as other on-board avionics and the payload electronics. Smaller lithium batteries are also used to provide auxiliary backup power. The avionics components consist of an autopilot system and a differential RTK GPS. The payload consists of a fluxgate magnetometer mounted at the front of the SkyLance UAS, used for aircraft attitude and navigation, and a cesium vapour magnetometer, which is used to perform the aeromagnetic surveys, is mounted at the end of a large pole on the back of the aircraft (hence the name SkyLance). The cesium vapour magnetometer can have a sampling frequency as high as 10 samples per second. A differential RTK GPS unit is used to provide positioning accuracy up to $5 \mathrm{~cm}$ in the horizontal plane. The SkyLance UAS measures ASL altitude with a barometric altimeter. Presently there is no on-board equipment to measure altitude AGL, however, there are plans to install a laser and/or a radar altimeter.

During flight, the SkyLance UAS follows pre-determined waypoints. It is also possible to provide additional waypoints on-the-fly, alter the flight path in real time, re-fly previous survey lines, or return to the take-off and landing zone. The on-board autopilot commands the UAS to approach a waypoint as closely as possible without 
exceeding the specified limits for maximum pitch $\left(20^{\circ}\right)$ and roll $\left(20^{\circ}\right)$ values. The constraints are in place to ensure the safety of the UAS and to prevent drastic flight path adjustments. Once the UAS passes a waypoint, it will continue onto the next waypoint. Similar to the Venturer UAS, excursions along a flight line are limited to no more than $30 \%$ of the survey line spacing in order to ensure good data quality and regular data coverage. The capability of the system to follow closely a pre-determined flight line is dependent on atmospheric conditions (i.e. wind speed) as well as the accuracy of the autopilot's tuning parameters; which are derived from computer modelling of the aircraft. If required, the operator may also take control of the UAS at any time. This is most likely to be implemented to ensure safe take-off and landing of the system, but also might be performed if weather conditions are deteriorating.

Similar to the Venturer UAS, the SkyLance UAS has also been designed with various features to ensure the safety of not only the aircraft but also the environment and other airspace users. This includes back up power for critical components as well as the ability to set up a "geo-fence" to limit the range of the UAS. Depending on the local conditions (i.e. topography, vegetation, weather, antenna configuration), the UAS is able to operate at a maximum range of $5 \mathrm{~km}$ using a UHF radio communication link. If the SkyLance UAS loses connection with the operator it will return to home base and automatically land.

The SkyLance UAS is extremely manoeuvrable; as a helicopter system, it is capable of rotating in flight while stationary (hovering), and also ascend or descend vertically; this allows for it to fly at very low altitudes (down to $2 \mathrm{~m}$ AGL). This would 
allow the SkyLance UAS to be able to replace ground magnetic survey teams when surveying over barren regions such as northern tundra, frozen lakes, desert, etc. The minimum altitude chosen for a specific survey, however, will depend on several factors: weather, topography, vegetation (to fly at $2 \mathrm{~m}$ AGL in a forested area cut lines would be required), and survey design requirements. The maximum altitude depends on air density, air temperature, and weight of the aircraft (including payload). To ensure safe take-off and landing, the UAS requires at a minimum, a small patch of flat smooth ground (grass, dirt, tarmac, etc.), with dimensions of approximately $10 \mathrm{~m} \mathrm{x}$ $10 \mathrm{~m}$. 
Table 2.2: SkyLance UAS specifications

\begin{tabular}{|l|l|}
\hline Pole length: & Up to $3.6 \mathrm{~m}$ \\
\hline Rotor Spacing: & Approx. 1m \\
\hline Crew size: & 2 people \\
\hline Mass: & Approx. 20kg \\
\hline Mass of Payload: & $\begin{array}{l}\text { 5 kg (includes magnetic } \\
\text { sensors, cameras, video, or } \\
\text { LIDAR) }\end{array}$ \\
\hline Endurance: & 30min at 35km/h \\
\hline $\begin{array}{l}\text { Maximum flight } \\
\text { speed: }\end{array}$ & 37km/h (air speed) \\
\hline GPS: & Differential GPS \\
\hline GPS Accuracy: & 5cm \\
\hline Maximum Range: & 5km \\
\hline Engine: & 8 battery powered rotors \\
\hline Power: & $\begin{array}{l}\text { Geometrics G-823A cesium } \\
\text { vapour magnetometer }\end{array}$ \\
\hline Sensor: & $\begin{array}{l}\text { Applied Physics model 539 } \\
\text { high speed digital 3-axis } \\
\text { magnetometer }\end{array}$ \\
\hline $\begin{array}{l}\text { Fluxgate } \\
\text { magnetometer: }\end{array}$
\end{tabular}




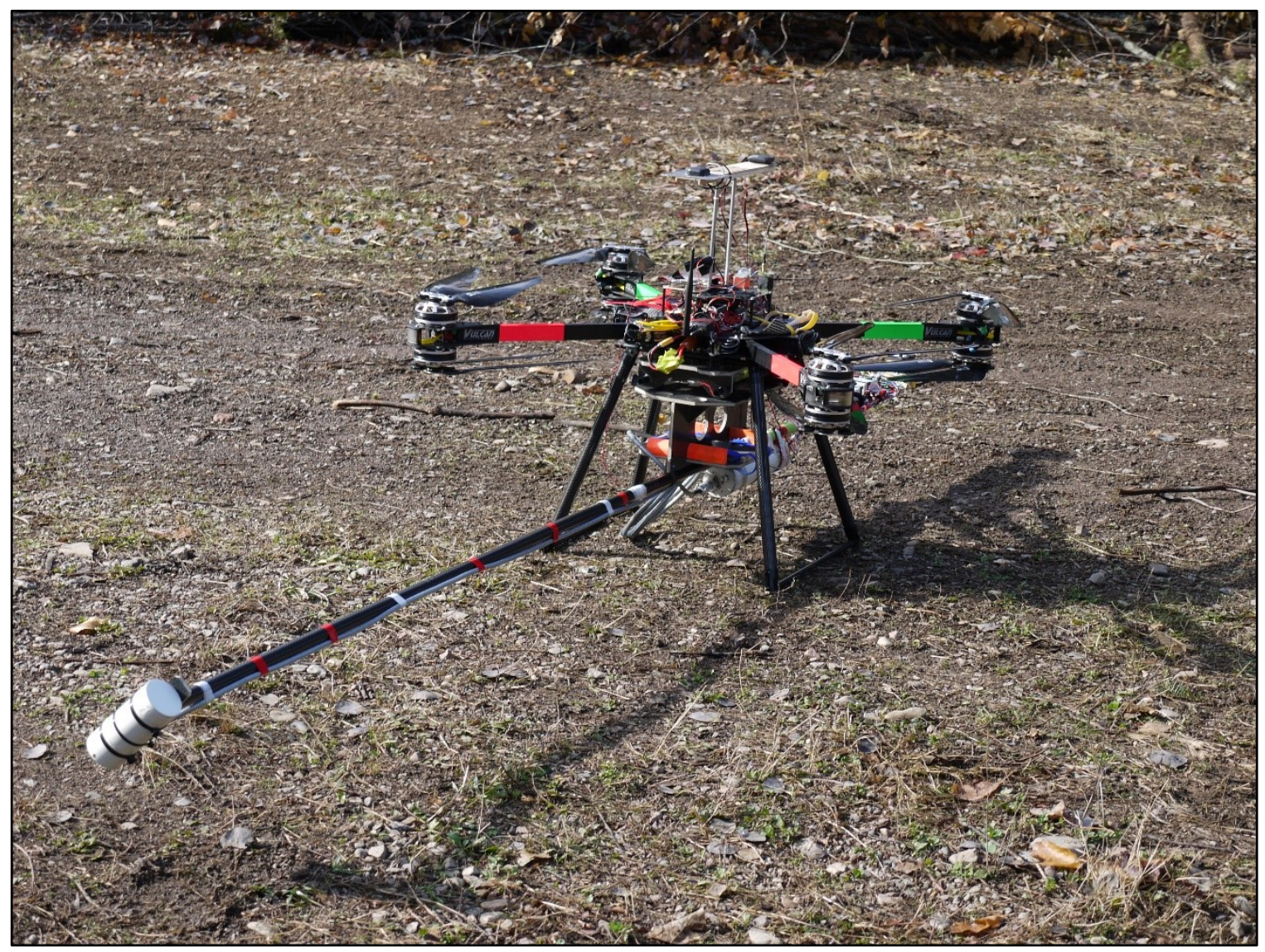

Figure 2.5: SkyLance rotary-wing UAS mounted with cesium vapour magnetometer on the end of a boom. 


\section{3 - UAS Theoretical Capabilities}

In the mineral exploration industry, UASs are believed to be eventually able to fulfill the demand for high-resolution, small area surveys that are flown at very low altitudes (Barnard, 2008). They are expected to be able to provide higher resolution data and to operate at lower costs compared to traditional airborne and ground survey systems (Kroll, 2013). The analyses presented in this chapter aim at assessing the capabilities of the UASs compared to traditional aeromagnetic survey systems, specifically for mineral exploration purposes. Three different systems were examined, representing different survey flight altitudes: a rotary-wing UAS, the SkyLance (2m AGL); a fixed-wing UAS, the Venturer (50m AGL); and a traditional fixed-wing aircraft (100m AGL).

The first part of the analysis was to determine the minimum detection limits of each system; minimum size of a body, minimum magnetic susceptibility, and maximum depth below ground level it can be and still be detectable by each system. To do this, the following parameters were considered: (1) the design parameters of each system; (2) mathematical models of spherical magnetic ore bodies; (3) a realistic noise level; and (4) spatial resolution. Further analysis investigated the gradiometry capabilities of the Venturer UAS compared to the traditional fixed-wing system. Lastly, the spatial resolution limit, that is, the minimum separation between two magnetic bodies for which both bodies can be detected individually within the resultant signal, of each system was determined. 
Design parameters were taken from the two UASs, the Venturer (Section 2.2.1), and the SkyLance (Section 2.2.2). The design parameters for the traditional fixed-wing aircraft were taken from a Cessna Grand Caravan, shown in Figure 3.1

The Cessna Grand Caravan carries two cesium vapour magnetometers mounted on each wingtip as well as two more magnetometers mounted vertically on a tail stinger. This allows for measurement of the TMI, and horizontal (transverse and in-line), and vertical gradients. The separation between the wingtip magnetometers is approximately $19 \mathrm{~m}$. Further details of the Cessna are provided in Table 3.1.

In Section 3.1 the magnetic model used for the analysis as well as the approach chosen are discussed. Section 3.2 provides the results of the three analyses and Section 3.3 is the overall discussion and conclusion. 


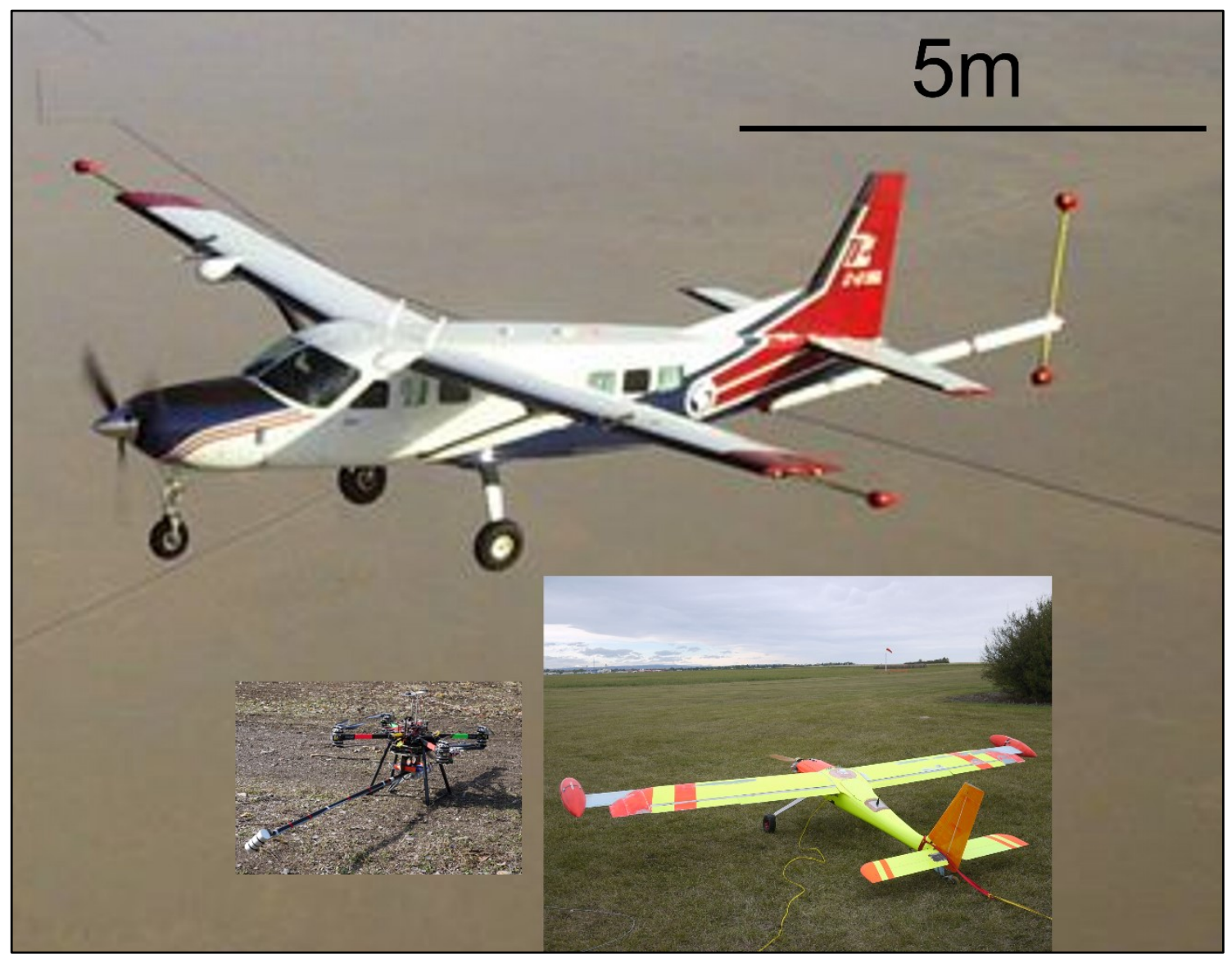

Figure 3.1: A scale comparison of the three aeromagnetic systems considered. The top picture is the Cessna Grand Caravan (courtesy of Sander Geophysics Ltd.). The bottom left is the SkyLance rotary-wing UAS. The bottom right is the Venturer fixed-wing UAS. 
Table 3.1: Specification of the Cessna Grand Caravan.

\begin{tabular}{|l|l|}
\hline Length: & $12.67 \mathrm{~m}$ \\
\hline Wingspan: & $19.03 \mathrm{~m}$ \\
\hline Crew size: & $2+$ people \\
\hline Mass: & Approx. $3995 \mathrm{~kg}$ \\
\hline Mass of Payload: & Variable \\
\hline Endurance: & $9-10 \mathrm{~h}$ at $200 \mathrm{~km} / \mathrm{h}$ \\
\hline Maximum flight speed: & 343km/h (air speed) \\
\hline Sensors: & $\begin{array}{l}\text { Four Geometrics cesium } \\
\text { vapour magnetometers }\end{array}$ \\
\hline
\end{tabular}




\section{1 - Magnetic Anomaly Modelling}

Here the spherical magnetic anomaly model used for the theoretical analysis, as well as the methods used for analysis are outlined.

\subsection{1 - Spherical Magnetic Anomaly}

To determine the aeromagnetic detection capabilities of each flight altitude and system, a magnetic anomaly needs to be considered. The magnetic spherical anomaly was selected for this analysis due to the simplicity of the derivation and calculations compared to that of other geological features (i.e. dykes, cylinders).

Modifying a derivation by Telford et al. (1976), a three dimensional mathematical model was determined to calculate the magnetic anomaly caused by a spherical ore body in the subsurface. The three Cartesian components, $B_{x}, B_{y}$, and $B_{z}$, of the magnetic field from the source body are described by Eq. 3.1, Eq. 3.2, and Eq. 3.3.

$$
\begin{aligned}
B_{x}=\frac{4 \pi R^{3} k}{3\left(x^{2}+y^{2}+z^{2}\right)^{5 / 2}}\left[H_{o} \sin (\beta)\left(2 x^{2}-y^{2}-z^{2}\right)-H_{o} \cos (\beta) 3 x y\right. \\
\left.+Z_{o} 3 x z\right] \\
B_{y}=\frac{4 \pi R^{3} k}{3\left(x^{2}+y^{2}+z^{2}\right)^{5 / 2}}\left[H_{o} \sin (\beta) 3 x y-H_{o} \cos (\beta)\left(-x^{2}+2 y^{2}-z^{2}\right)\right. \\
\left.+Z_{o} 3 x z\right]
\end{aligned}
$$




$$
\begin{gathered}
B_{z}=\frac{4 \pi R^{3} k}{3\left(x^{2}+y^{2}+z^{2}\right)^{5 / 2}}\left[H_{o} \sin (\beta) 3 x z-H_{o} \cos (\beta) 3 y z\right. \\
\left.+Z_{o}\left(-x^{2}-y^{2}+2 z^{2}\right)\right]
\end{gathered}
$$

Variables $H_{o}$ and $Z_{o}$ are the horizontal and vertical components of the external Earth's magnetic field respectively, $\beta$ is the declination of the Earth's magnetic field, $k$ is the magnetic susceptibility of the body, $R$ is the body radius, and $x, y$, and $z$ are the spatial coordinates of the location in space where the magnetic field is being calculated. The $x$ and $y$ coordinates represent the horizontal position of the location of interest, and the $z$ coordinate represents vertical position. For the analyses presented in this paper, the vertical coordinate is the sum of the flight altitude (FA) of the survey system under consideration and the limiting depth of the spherical body (LD) (Eq. 3.4). Substituting Eq. 3.1, Eq. 3.2, and Eq. 3.3, into Eq. 3.5 the TMI, $B_{T}$, can be calculated.

$$
\begin{gathered}
z=z_{L D}+z_{F A} \\
B_{T}=\sqrt{B_{x}^{2}+B_{y}^{2}+B_{z}^{2}}
\end{gathered}
$$

In most cases, there is also a demagnetization effect which results in a reduction of the magnetic field. Depending on the shape of the magnetic body, the induced magnetic field is not exactly the product of $k$. Generally there is a demagnetizing effect that reduces the resultant magnetic field within the body. The demagnetization factor is $D$ : 


$$
D=\frac{1}{1+N k} \text { where }(0 \leq N \leq 4 \pi)
$$

where $N$ varies depending on the shape of the body under consideration. No demagnetization occurs for a rod-like body with magnetization along the long-axis and with a small cross-section compared to its length. A maximum demagnetization occurs for a thin sheet that is magnetized in a direction normal to its surface. For a spherical body, $N=4 \pi / 3$, the resultant TMI is:

$$
B_{T D}=D \cdot B_{T}
$$

An example profile plot of anomalies due to a spherical body in the subsurface is presented in Figure 3.2 for the three survey altitudes of interest. Since the TMI falls off as a function of inverse distance cubed, signal strength decreases markedly when flight altitude increases. Note that the analyses presented in this thesis are performed only on residual magnetic anomalies (i.e. regional effects are ignored) (Li and Oldenburg, 1998). 


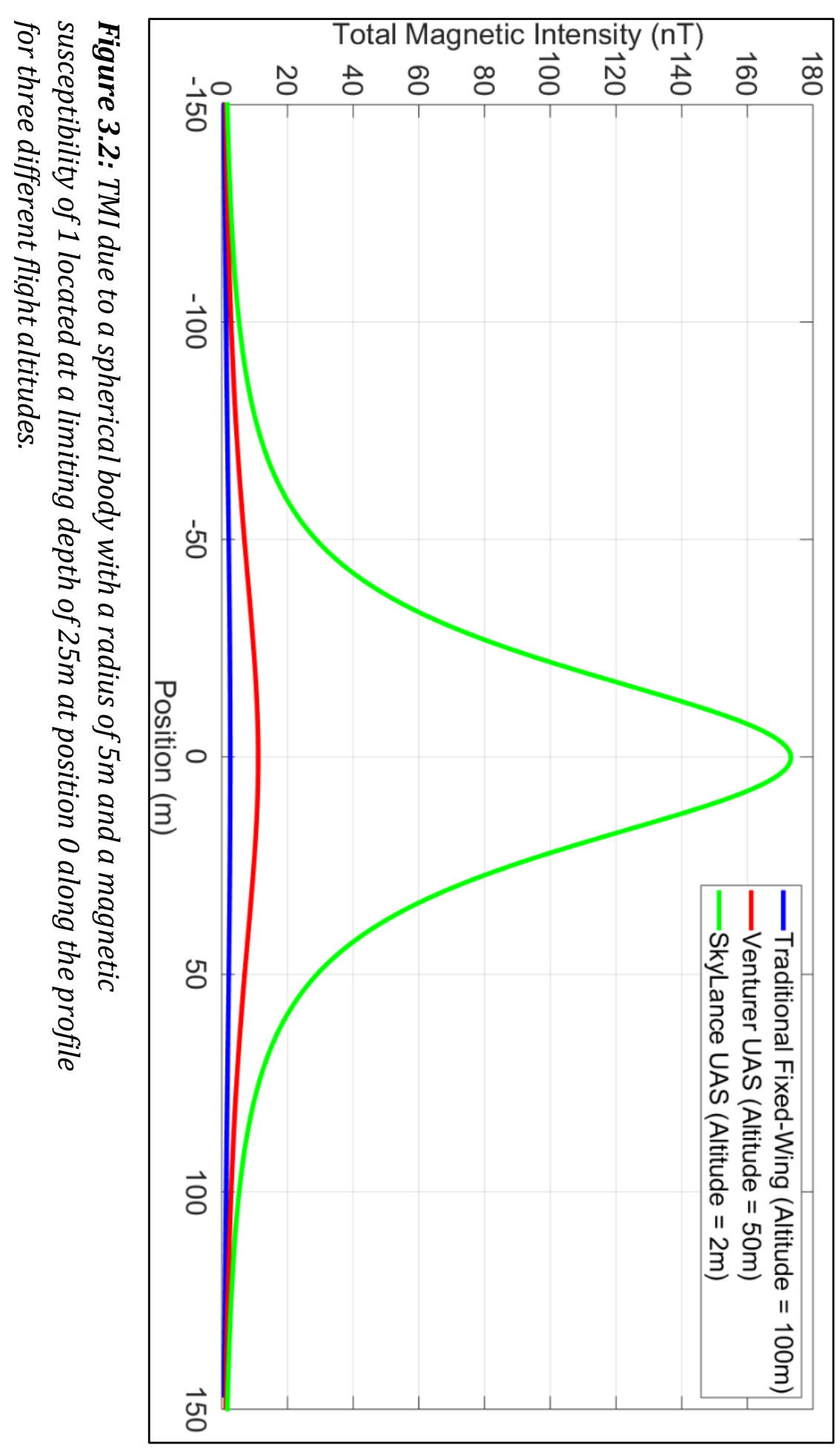




\subsection{2 - Anomaly Detection}

A method for determining whether or not an anomaly is detectable above noise was required. Aeromagnetic noise is primarily composed of two sources: highfrequency continuous noise due to flexing of the aircraft and spikes due to measurement errors and the presence of nearby instrumentation onboard the aircraft (Teskey et al. 1991). Aeromagnetic noise was characterized using data from a test flight of the Venturer UAS done on October 3, 2013 (Wood, A., et al., 2016) to provide a "worst case" scenario for each flight altitude. Through the use of long wavelength filters, regional and geological effects were filtered out so that only noise from both flexing and spikes remained. Binning the filtered data from two survey lines showed that it follows a normal distribution centered at 0nT and with a standard deviation of $0.059 \mathrm{nT}$ (Figure 3.3). For analyses to follow, random noise following this distribution was added to synthetic data.

The spatial sampling interval, which is a function of flight speed and the sampling frequency of the data acquisition systems onboard the aircraft, also has an effect on anomaly detection. The spatial sampling intervals for the three survey systems of interest are provided in Table 3.2. Small anomalies will go undetected if the spatial sampling interval is too coarse. 


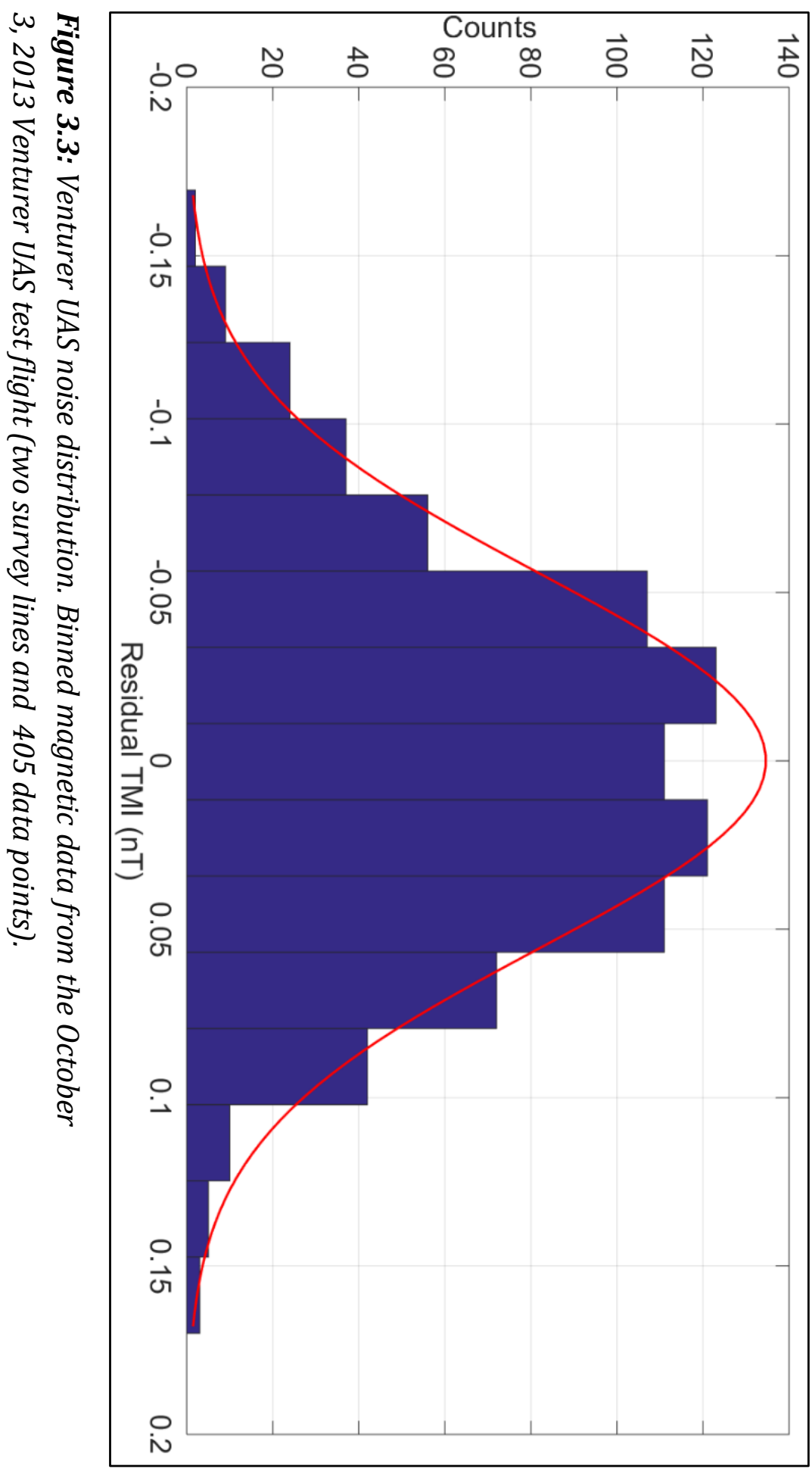


Table 3.2: Flight speed and spatial sampling intervals for different survey systems. Spatial sampling intervals have been calculated for a $10 \mathrm{~Hz}$ sampling frequency.

\begin{tabular}{|c|c|c|c|}
\hline & $\begin{array}{c}\text { Traditional Fixed- } \\
\text { Wing (100m) }\end{array}$ & $\begin{array}{c}\text { Venturer UAS } \\
\text { (50m) }\end{array}$ & $\begin{array}{c}\text { SkyLance UAS } \\
\text { (2m) }\end{array}$ \\
\hline $\begin{array}{c}\text { Flight Speed } \\
\text { (km/h) }\end{array}$ & 200 & 100 & 32 \\
\hline $\begin{array}{c}\text { Spatial Sampling } \\
\text { Interval (m) }\end{array}$ & 5.6 & 2.8 & 0.9 \\
\hline
\end{tabular}


A statistical testing method was employed in order to consider whether an anomaly could be successfully detected. Statistical testing does not rely on the selection of arbitrary criteria such as requiring a fixed number of points above the noise level for a successful detection. Anomalous data points are also not required to be consecutively detected, which makes the approach robust even in the presence of noise. Furthermore, statistical testing provides a quantitative metric indicating how likely the anomaly will be successfully detected. The statistical method employed here is the chi-squared $\left(\chi^{2}\right)$ goodness-of-fit (GOF) test. The test determines the overall discrepancy between observed (O) and expected (E) signals, which is given as the $\chi^{2}$ value (Johnson and Bhattacharyya, 2014):

$$
\chi^{2}=\sum_{N}^{i=1} \frac{\left(O_{i}-E_{i}\right)^{2}}{E_{i}}
$$

If the $\chi^{2}$ value is less than a threshold, $\chi_{1-\alpha}^{2}$, then the observed data is considered to fit the expected result. $\chi_{1-\alpha}^{2}$ is determined by using the probability density curve of a $\chi^{2}$ distribution. This curve varies depending on the number of degrees of freedom, N. For a significance level, $\alpha$, and a number of degrees of freedom, $\mathrm{N}$, the $\chi_{1-\alpha}^{2}$ is the value where $100(1-\alpha)$ percent of the data is contained.

For the analyses performed in this paper, the observed signal is a combination of the TMI from a spherical body plus randomly generated noise following the distribution shown in Figure 3.3. The expected signal is simply the noise-free TMI from the same spherical body. The number of degrees of freedom is equal to the number of data points in the signal. The required significance level has been set to 
0.01 . This value was selected instead of a significance level of 0.05 in order to reduce the likelihood that the $\chi^{2}$ GOF test might reject the observed signal when it should pass the test, reducing the possibility of a type 1 statistical error (Peck \& Devore, 2012).

For a specific set of randomly generated noise, it is possible that the data will successfully pass the $\chi^{2}$ test but for the next set of randomly generated noise, the data might fail the test. By performing $\chi^{2}$ tests multiple times for an anomaly generated using a specific set of parameters but with different random noise, it is possible to determine the likelihood that the observed signal will pass the test. An example of two sets of magnetic noise added to the same magnetic anomaly model is shown in Figure 3.4; here the top plot will pass the $\chi^{2}$ GOF whereas the bottom plot does not. Furthermore, by varying one of the model parameters slightly, such as the body radius, a cumulative distribution function can be created and used to select an acceptable anomaly detection success rate. Figure 3.5 shows success rate versus the radius of the sphere that was used to generate the anomaly. Each data point represents how many successes (out of 100 tests) occurred for a specific body radius. By fitting a sigmoid curve to this data, the radius required for a desired success rate (i.e. a $90 \%$ success rate corresponds to a radius of $5.32 \mathrm{~m}$ ) can be determined. By performing repeated analyses over a wide range of body radii, limiting depths and magnetic susceptibilities, a set of minimum detection parameters for each desired success rate is created. 


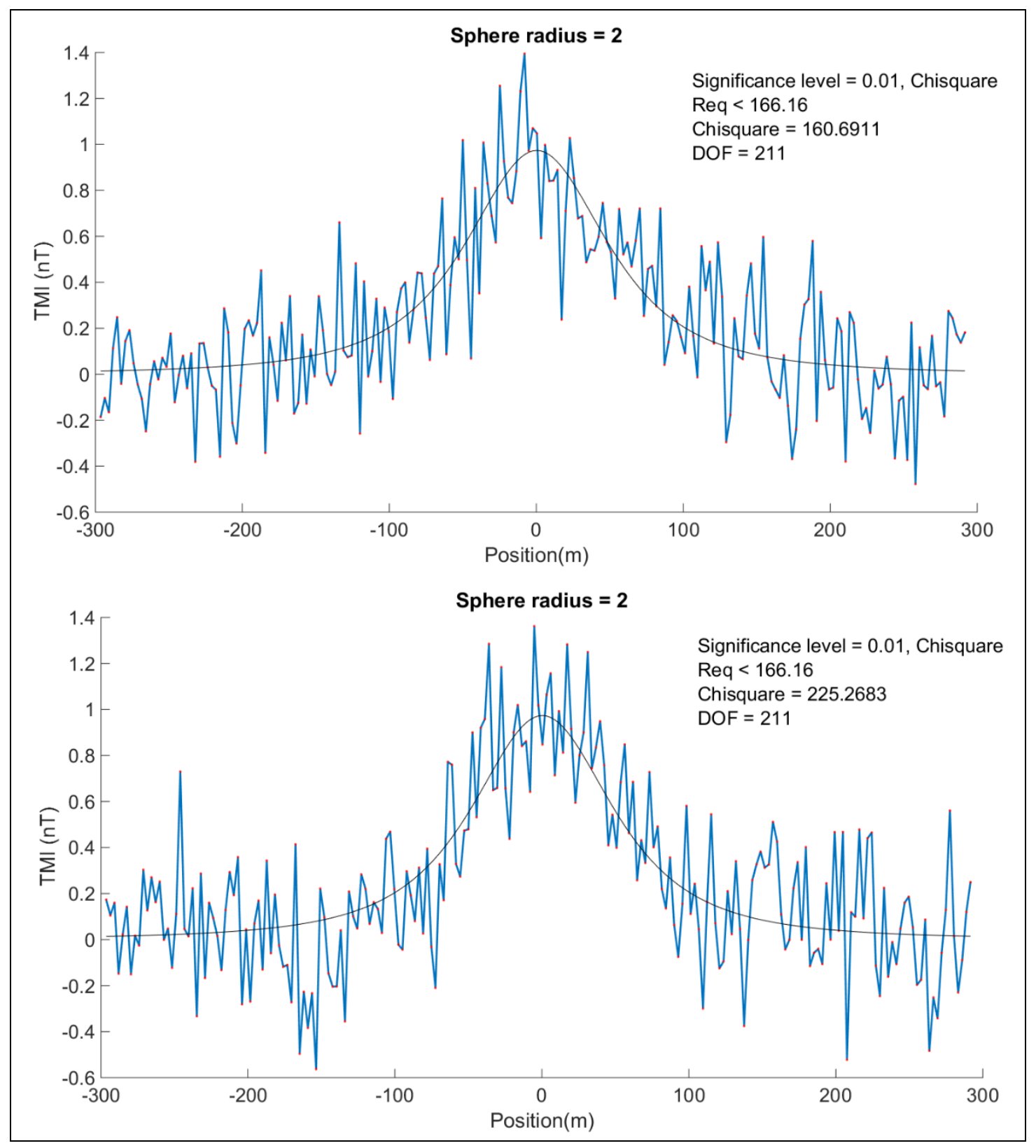

Figure 3.4: $\chi^{2}$ GOF test comparison. Two observed datasets that contain the same anomaly signal but different noise levels. The $\chi^{2}$ value needs to be below a value of 166. The top plot has a value of 161, so it passes the $\chi^{2}$ GOF test. However, the bottom has a $\chi^{2}$ value of 225, so it fails the $\chi^{2}$ GOF test. 


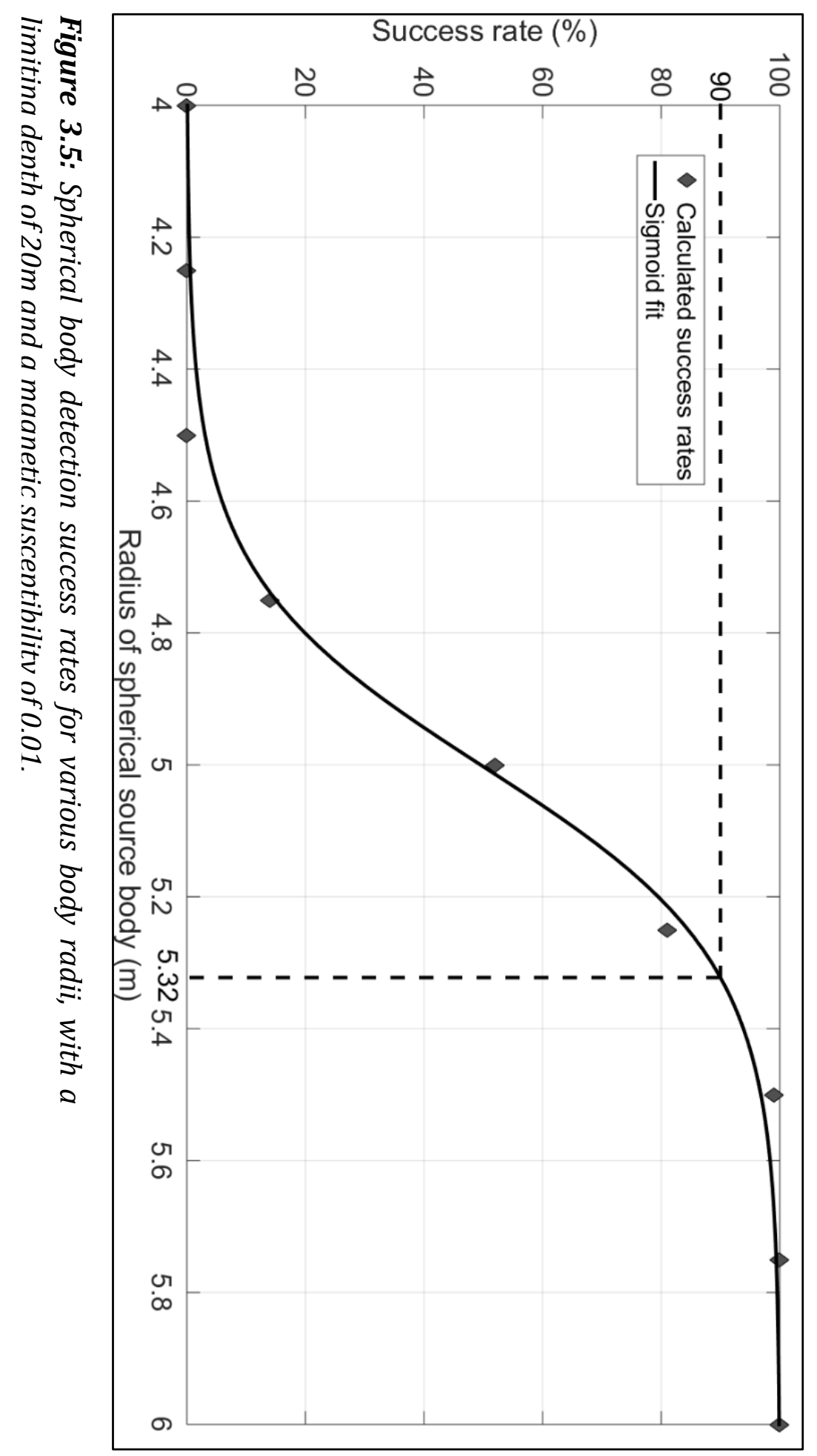




\subsection{3 - Gradiometry Capabilities}

An understanding of how gradiometry with smaller aircraft, such as UASs, would perform in comparison to the traditional aeromagnetic gradiometry systems was sought. Due to their small size, UASs have a smaller magnetometer separation; the signal recorded by the two magnetometers is almost the same, potentially reducing the effectiveness of gradiometry. On the other hand, they fly closer to the ground, which increases signal strength (Figure 3.2), potentially improving gradiometry capabilities.

The gradiometry analysis presented in Section 3.2.2 focusses only on transverse gradiometry for two flight altitudes AGL; 100m (the traditional survey system) and 50m (the fixed-wing UAS). This technique enhances subtle features varying in the direction of flight (Keary et al., 2002). Figure 3.6 shows a TMI map with a spherical anomaly and Figure 3.11 (bottom) provides the corresponding transverse gradient.

Through error propagation, the error on gradiometric data due to the error from each magnetometer can be calculated using (see Appendix A for the derivation):

$$
\delta \frac{\partial B}{\partial x}=\sqrt{2} \frac{\delta B}{\Delta x}
$$

where $\delta \frac{\partial B}{\partial x}$ is the error on the magnetic gradient and $\delta B$ is the error from each magnetometer. This relationship is shown in Figure 3.7 for the noise level, $\delta B=$ $0.059 n T$, discussed in Section 3.1.2. Figure 3.7 reveals that the error curve becomes almost flat for a magnetometer separation of $5 \mathrm{~m}$ or more. The error on gradiometric 
data for the Venturer UAS is approximately 4 times larger than that of a traditional fixed-wing aircraft. 


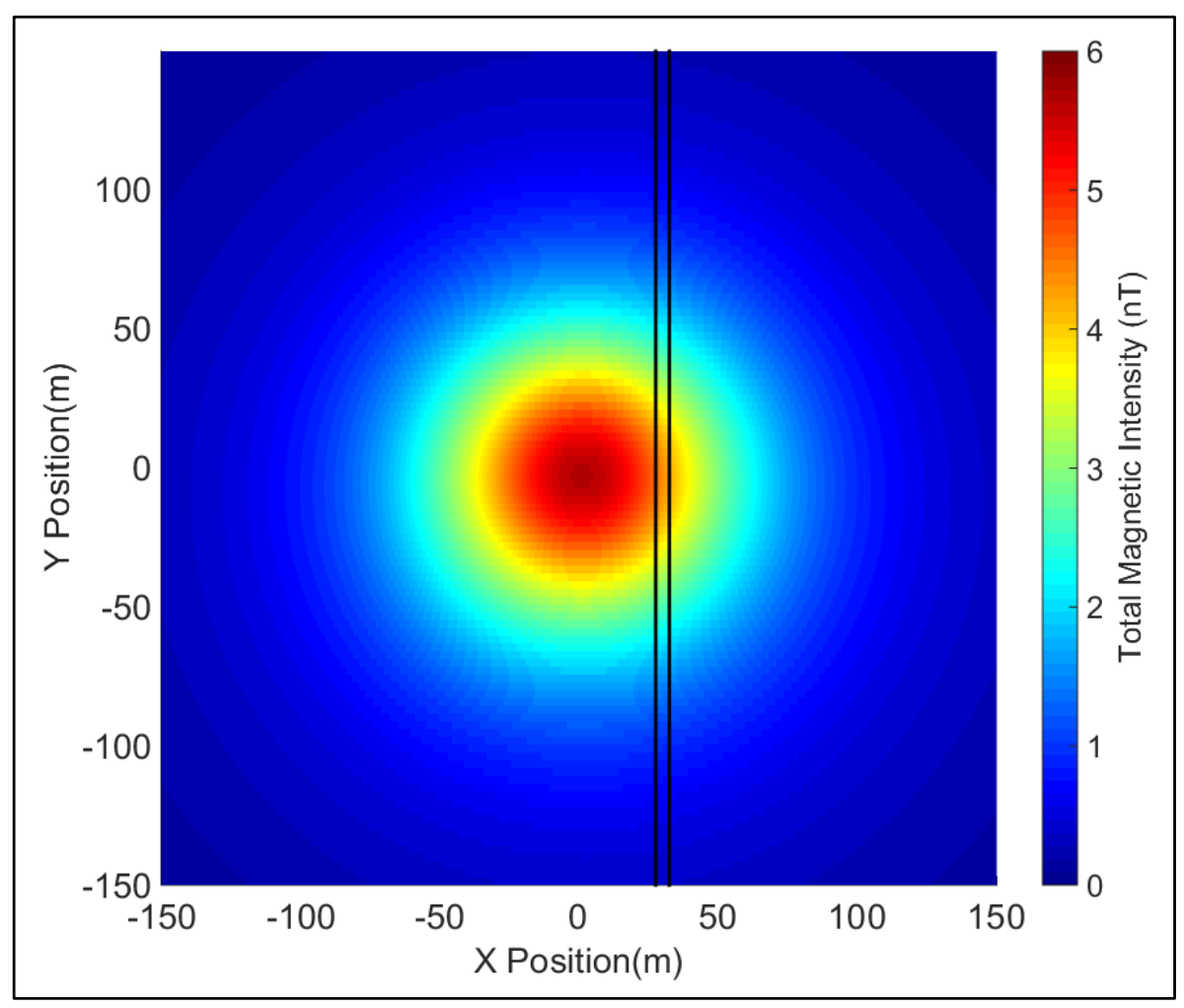

Figure 3.6: TMI map with a spherical anomaly. Two black lines represent locations of each magnetometer as the aircraft flies parallel to the y-axis in order to record gradiometric data. In this example, the magnetometers are separated by $4.95 \mathrm{~m}$, the wingspan of the Venturer UAS. 


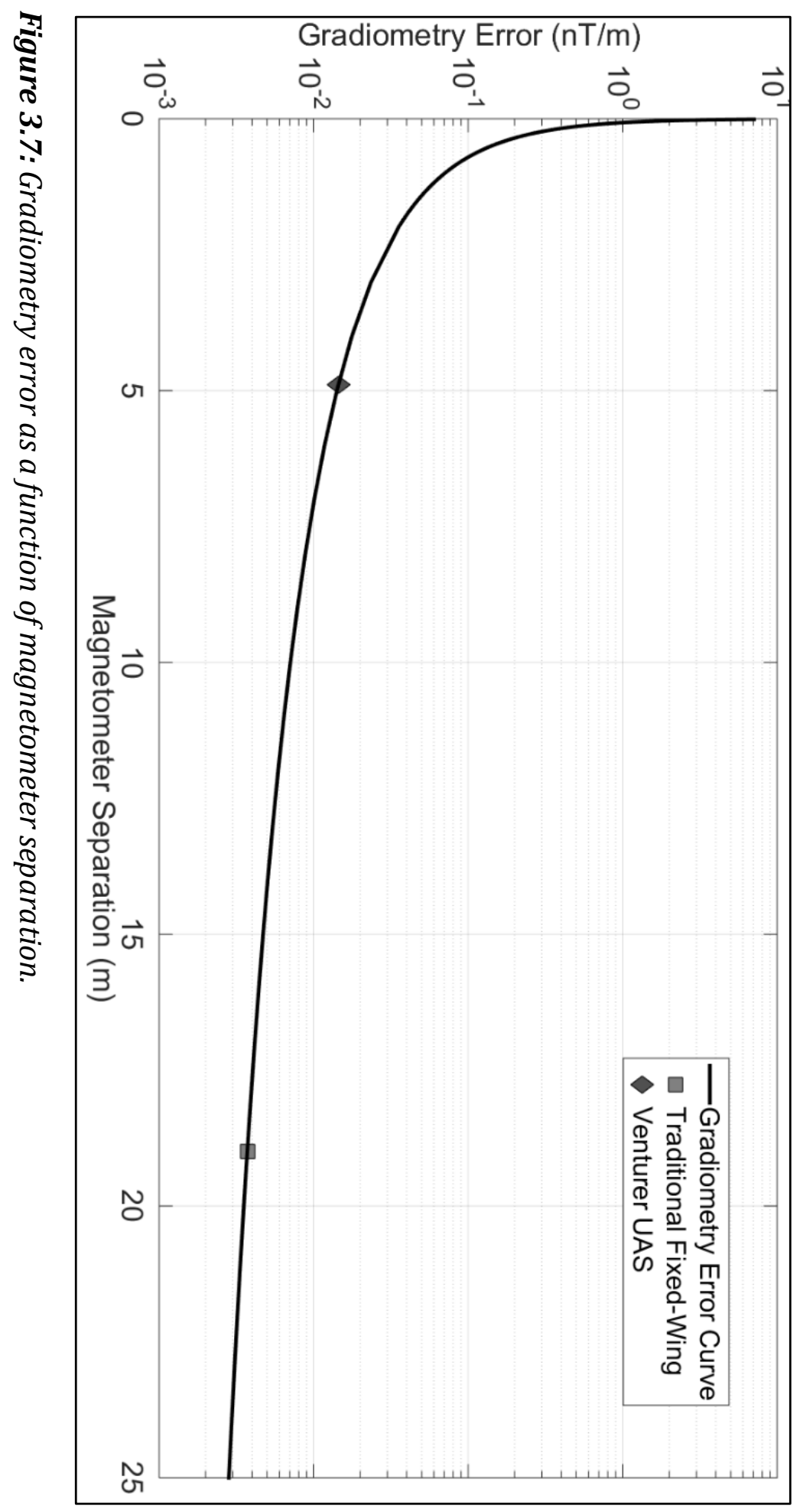




\subsection{4 - Spatial Resolution}

For each survey system there is a spatial resolution limit. This is the minimum separation between two bodies in the subsurface for which it is possible to differentiate that two bodies are contributing to the observed signal (Elkins and Hammer, 1938). Two bodies that have a large enough separation will create two distinct peaks in the magnetic data. As the bodies are brought closer, the signals from each body will progressively blend together. As shown in Figure 3.8, the blue signal exhibits a prominent double peak; it is obvious that it is caused by two separate bodies. For the red signal, however, the peaks have merged and it is not impossible to ascertain if there is one or two source bodies.

In order to mathematically determine the spatial resolution limit, the following conditions must be met (Elkins and Hammer, 1938):

1. Resolution is only considered for profiles that are produced from two identical bodies, in our case, two spherical bodies.

2. Each body can be represented by a symmetric function, $\phi(s)$, where $s$ is a horizontal coordinate along the profile under consideration. The origin at $\mathrm{s}=0$ corresponds to the axis of symmetry.

3. At the point $x=a$ (and $x=-a$ ), where $a$ is the location of the center of one of the bodies along the profile, $\phi(x)$ is analytical. Variable $x$ is the horizontal coordinate along the profile. The origin, $x=0$, is located halfway between the two bodies. 
The TMI is the sum of the contribution from each sphere over all $x$ :

$$
\Psi(x)=\Phi(x+a)+\Phi(x-a)
$$

To compute the spatial resolution limit, the second derivative of the $\Phi(x)$ function needs to be determined and set it equal zero and set $x=a$. Solving for $a$ provides half the resolution limit. This is because each body will be offset from the origin by an amount $a$, thus the total separation between each body is $2 a$. At a separation smaller than $2 a$ it will not be possible to distinguish the magnetic signature from the two bodies. 


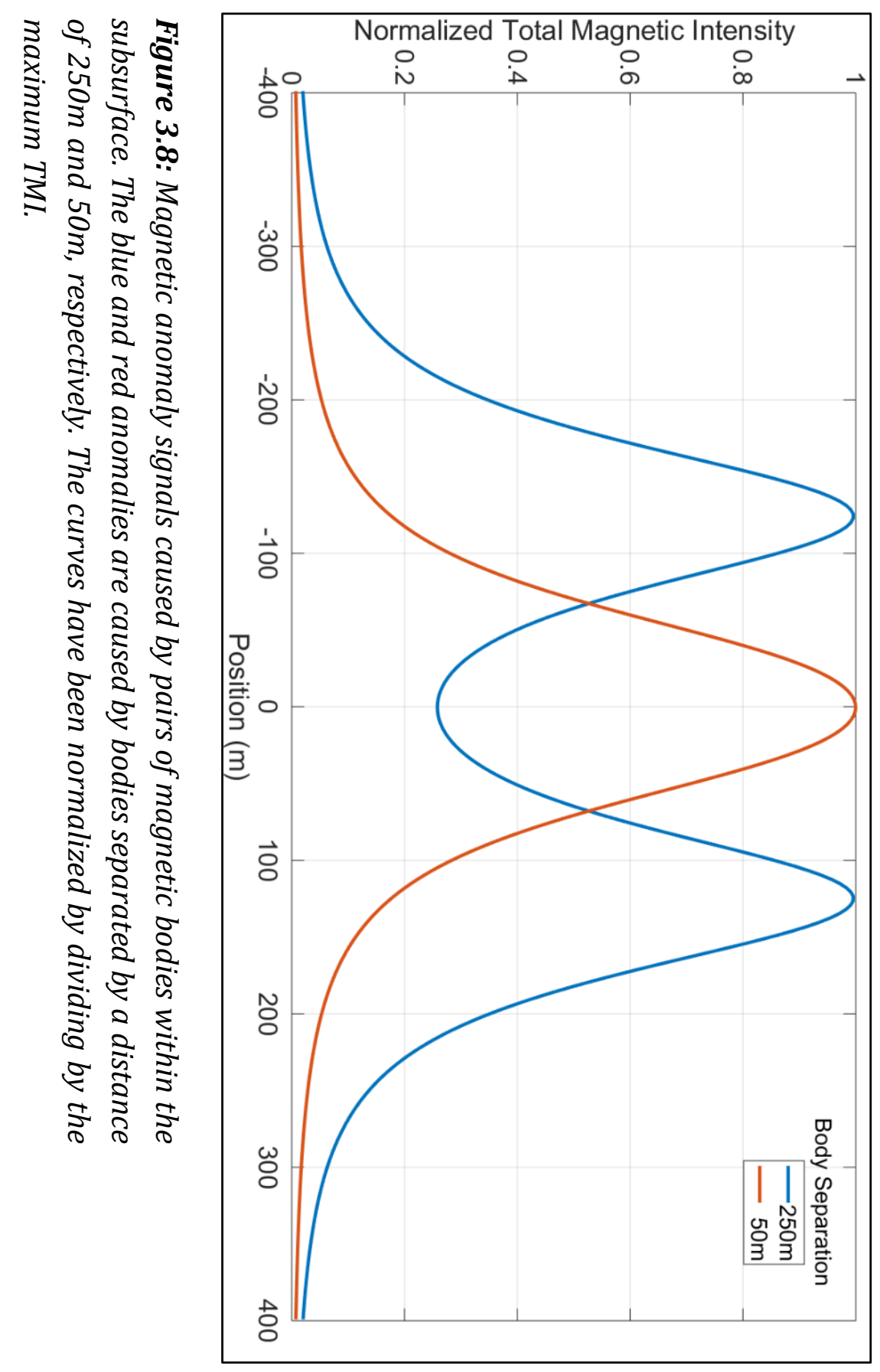


For the purposes of this paper, the TMI from a spherical body (equation 7) is considered as $\Phi(x)$ which fits the three requirements described by Elkins and Hammer (1938):

$$
\Phi(x)=B_{T D}(x)
$$

The second derivatives of the magnetic response are required (see Appendix $\mathrm{B}$ for the first derivatives of the magnetic response). The second derivative of $B_{T}$ is:

$$
\begin{aligned}
& \frac{d^{2}}{d x^{2}}\left(B_{T}\right) \\
& =\frac{B_{x} \frac{d^{2}}{d x^{2}}\left(B_{x}\right)+\left(\frac{d}{d x}\left(B_{x}\right)\right)^{2}+B_{y} \frac{d^{2}}{d x^{2}}\left(B_{y}\right)+\left(\frac{d}{d x}\left(B_{y}\right)\right)^{2}+B_{z} \frac{d^{2}}{d x^{2}}\left(B_{z}\right)+\left(\frac{d}{d x}\left(B_{z}\right)\right)^{2}}{\sqrt{B_{x}^{2}+B_{y}^{2}+B_{z}^{2}}} \\
& -\frac{\left(B_{x} \frac{d}{d x}\left(B_{x}\right)+B_{y} \frac{d}{d x}\left(B_{y}\right)+B_{z} \frac{d}{d x}\left(B_{z}\right)\right)\left(2 B_{x} \frac{d}{d x}\left(B_{x}\right)+2 B_{y} \frac{d}{d x}\left(B_{y}\right)+2 B_{z} \frac{d}{d x}\left(B_{z}\right)\right)}{\left(B_{x}^{2}+B_{y}^{2}+B_{z}^{2}\right)^{3 / 2}}
\end{aligned}
$$

with

$$
\begin{aligned}
\frac{d^{2}}{d x^{2}}\left(B_{x}\right)= & \frac{35 x^{2}\left(H_{o} \sin (\beta)\left(2 x^{2}-y^{2}-z^{2}\right)-3 H_{o} \cos (\beta) x y+3 Z_{o} x z\right.}{\left(x^{2}+z^{2}+z^{2}\right)^{9 / 2}} \\
& -\frac{10 x\left(4 H_{o} \sin (\beta) x-3 H_{o} \cos (\beta) y+3 Z_{o} z\right.}{\left(x^{2}+z^{2}+z^{2}\right)^{7 / 2}} \\
& -\frac{5\left(H_{o} \sin (\beta)\left(2 x^{2}-y^{2}-z^{2}\right)-3 H_{o} \cos (\beta) x y+3 Z_{o} x z\right)}{\left(x^{2}+z^{2}+z^{2}\right)^{7 / 2}} \\
& +\frac{4 H_{o} \sin (\beta)}{\left(x^{2}+z^{2}+z^{2}\right)^{5 / 2}}
\end{aligned}
$$




$$
\begin{array}{r}
\frac{d^{2}}{d x^{2}}\left(B_{y}\right)=\frac{35 x^{2}\left(3 H_{o} \sin (\beta) y x-H_{o} \cos (\beta)\left(-x^{2}+2 y^{2}+z^{2}\right)+3 Z_{o} z y\right)}{\left(x^{2}+z^{2}+z^{2}\right)^{9 / 2}} \\
-\frac{5\left(3 H_{o} \sin (\beta) y x-H_{o} \cos (\beta)\left(-x^{2}+2 y^{2}+z^{2}\right)+3 Z_{o} z\right)}{\left(x^{2}+z^{2}+z^{2}\right)^{7 / 2}} \\
-\frac{10 x\left(3 H_{o} \sin (\beta) y+2 H_{o} \cos (\beta) y\right)}{\left(x^{2}+z^{2}+z^{2}\right)^{7 / 2}}+\frac{2 H_{o} \cos (\beta)}{\left(x^{2}+z^{2}+z^{2}\right)^{5 / 2}} \\
\frac{d^{2}}{d x^{2}}\left(B_{z}\right)=\frac{35 x^{2}\left(3 H_{o} \sin (\beta) z x-3 H_{o} \cos (\beta) y z+Z_{o}\left(-x^{2}-y^{2}+2 z^{2}\right)\right)}{\left(x^{2}+z^{2}+z^{2}\right)^{9 / 2}} \\
-\frac{5\left(3 H_{o} \sin (\beta) z x-H_{o} \cos (\beta) y z+Z_{o}\left(-x^{2}-y^{2}+2 z^{2}\right)\right)}{\left(x^{2}+z^{2}+z^{2}\right)^{7 / 2}} \\
-\frac{10 x\left(3 H_{o} \sin (\beta) z+2 Z_{o} x\right)}{\left(x^{2}+z^{2}+z^{2}\right)^{7 / 2}}-\frac{2 Z_{o}}{\left(x^{2}+z^{2}+z^{2}\right)^{5 / 2}}
\end{array}
$$




\section{2 - Results}

Following the methods laid out in previous sections, minimum detection limits were determined for spherical bodies with a wide range of magnetic susceptibility, body radius, and limiting depth values. Further comparisons between UASs and traditional aircraft are presented for gradiometry and spatial resolution.

\subsection{1 - Minimum Detection Limits}

The minimum detection limits of a magnetic anomaly due to a spherical body were determined for the three survey systems of interest. For the analyses performed, magnetic susceptibility varied between $10^{-5}$ and $10^{4}$, the radius between $0 \mathrm{~m}$ and $30 \mathrm{~m}$, and the limiting depth between 0 and $100 \mathrm{~m}$. The traditional Cessna fixed-wing aircraft, Venturer fixed-wing UAS and the SkyLance rotary-wing UAS had a flight altitude set at $100 \mathrm{~m}, 50 \mathrm{~m}$, and $2 \mathrm{~m}$, respectively. Each system used normally distributed random noise with the same standard deviation of $0.059 \mathrm{nT}$.

Figure 3.9 shows surfaces representing the minimum values for each body parameter for which the corresponding magnetic anomaly will be detectable. Detection corresponds to a success rate of $90 \%$ or higher in the $\chi^{2}$ test. For large body radii and/or small limiting depths, the magnetic susceptibility can be as low as the order of $10^{-5}$ and the body will be considered detectable. As the radius decreases and/or the limiting depth increases, magnetic susceptibility must increase for a successful detection. 
The results can be also displayed as profile plots for different success rates. Figure 3.9 show the effects of varying 2 body parameters, while the third parameter is kept constant, for $50 \%$ and $90 \%$ success rates. The top plot, the source body is set to have a limiting depth of $20 \mathrm{~m}$ and the relationship between the body radius and magnetic susceptibility is shown. Any set of values above and to the right of a curve is considered detectable. In order to continue to successfully detect the body as its radius decreases, the magnetic susceptibility must increase rapidly. In the middle plot of Figure 3.10, the relation between the limiting depth and magnetic susceptibility for a body with a radius set to $10 \mathrm{~m}$ is shown. Anything above the curves is considered detectable. As limiting depth increases, the magnetic susceptibility also needs to increase for a successful detection. Lastly, the bottom plot presents the relationship between the limiting depth and radius of the spherical body when the magnetic susceptibility is held constant at $10^{-4}$. A linear relationship between the limiting depth and radius is observed; anything to the right of the curves is considered detectable. As the limiting depth increases, the radius of the body must also increase for the body to be detectable.

Figure 3.10 clearly shows the superior detectability afforded by UASs. A UAS flying at an altitude of $50 \mathrm{~m}$ will detect a weakly magnetic $\left(10^{-4}\right)$ ore body with a radius of $10 \mathrm{~m}$ buried at a limiting depth of approximately $30 \mathrm{~m}$. A UAS flying at an altitude of $2 \mathrm{~m}$ will detect the same body at a limiting depth of approximately $80 \mathrm{~m}$. The body will not be detected by the traditional fixed-wing aircraft. Figure 3.9 also reveals that improvements in detectability are almost the same when lowering the flight altitude from to $100 \mathrm{~m}$ to $50 \mathrm{~m}$, and from $50 \mathrm{~m}$ to $2 \mathrm{~m}$, due to the fact the TMI falls off as a 
function of inverse distance cubed. In practice, flying a middle-size UAS such as the Venturer UAS at an altitude slightly higher or lower than its nominal flight altitude will have little effect overall. 


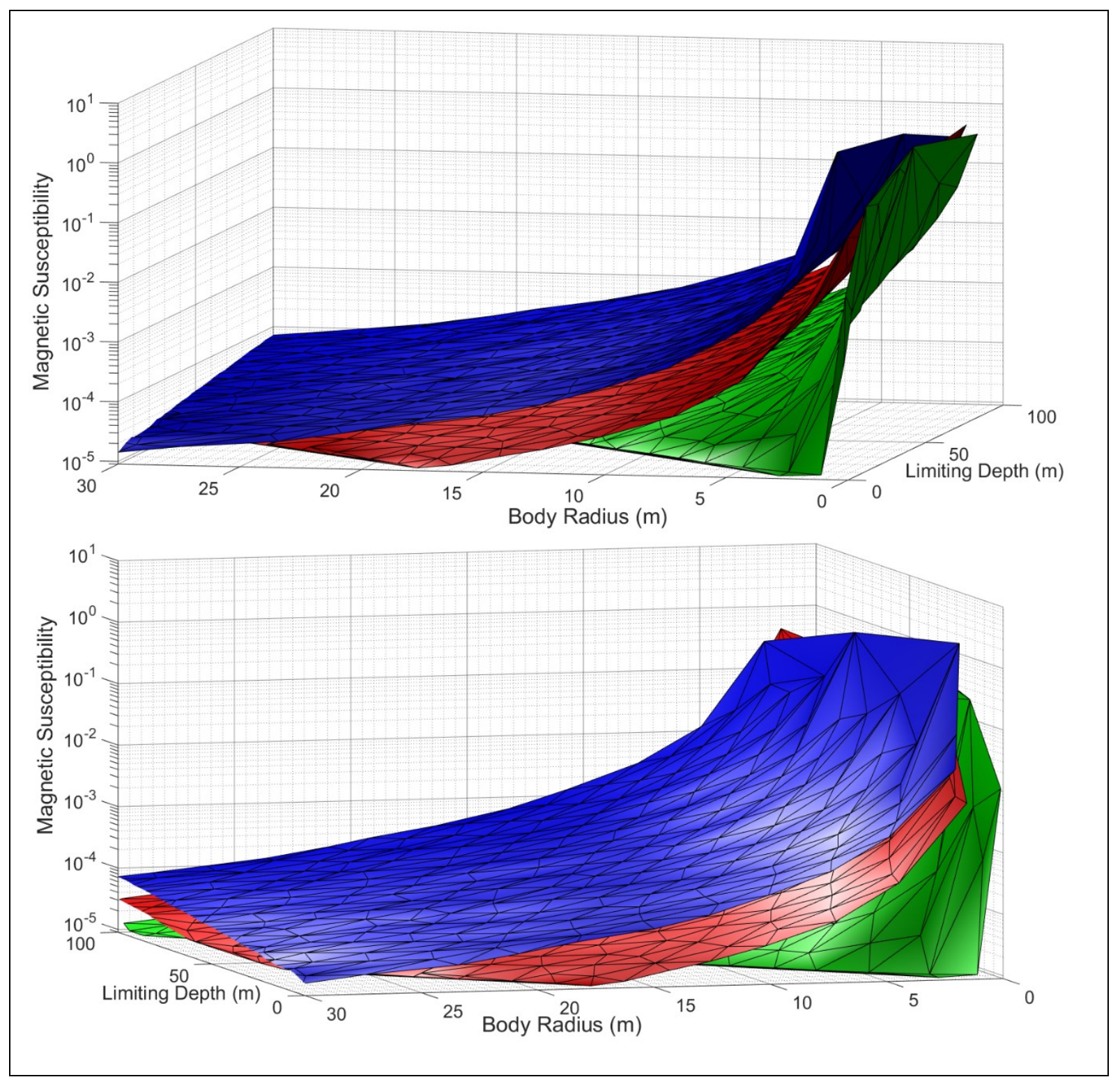

Figure 3.9: Surface plots of the minimum detection limits with a 90\% success rate for each survey system with respect to the magnetic susceptibility, body radius, and limiting depth. Blue $-100 \mathrm{~m}$ flight altitude (i.e. Traditional Fixed-wing system); Red $-50 \mathrm{~m}$ flight altitude (i.e. Venturer UAS); and Green $-2 m$ flight altitude (i.e. SkyLance UAS). See Appendix $C$ for individual figures of each surface. 


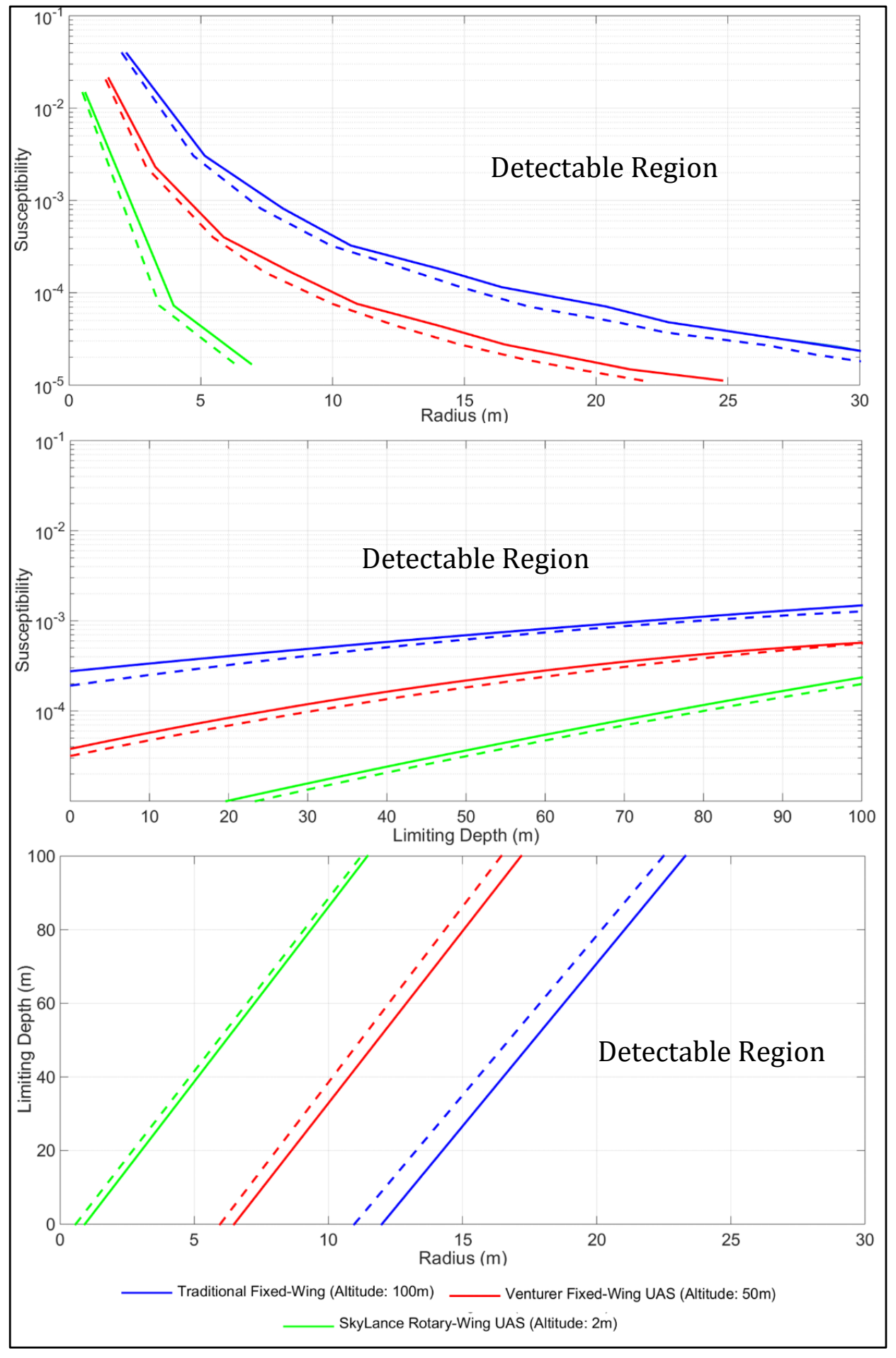

Figure 3.10: Minimum detection limits for a magnetic body. The top plot is for a limiting depth of $20 \mathrm{~m}$ and the middle plot is for body radius of $10 \mathrm{~m}$. The detectable region is above the curves. The bottom plot is for a magnetic susceptibility of $10^{-4}$. The detectable region is right of the curves. The solid and dashed lines represent $90 \%$ and $50 \%$ success rates, respectively. 


\subsection{2 - Gradiometry Capabilities}

The detectable region on a gradiometric map can be outlined by requiring the magnetic gradient to be greater than a certain noise threshold. It then becomes possible to assess the performance of different systems by comparing the size of the detectable regions on their respective gradiometric maps.

A transverse gradiometry map for the anomaly shown in Figure 3.6 was produced for the traditional fixed-wing aircraft flying at 100m AGL and the Venturer UAS flying at 50m AGL (Figure 3.11). The scales of each map are an order of magnitude different due to the different separation distance between the magnetic sensors. The black contour lines encompass the detectable region, which is defined as the area where the signal strength is above the noise level; $\pm 0.0037 \mathrm{nT} / \mathrm{m}$ for the traditional fixed-wing and $\pm 0.014 \mathrm{nT} / \mathrm{m}$ for the Venturer UAS. The detectable regions are $27,911 \mathrm{~m}^{2}$ for the traditional fixed-wing and $22,167 \mathrm{~m}^{2}$ for the Venturer UAS. Figure 3.11 shows that the performance of the two systems for the acquisition of transverse gradiometric magnetic data is very similar. Any adverse effect due to the small separation between the magnetometers mounted on the Venturer UAS has been compensated by its ability to fly lower and record a stronger signal. 


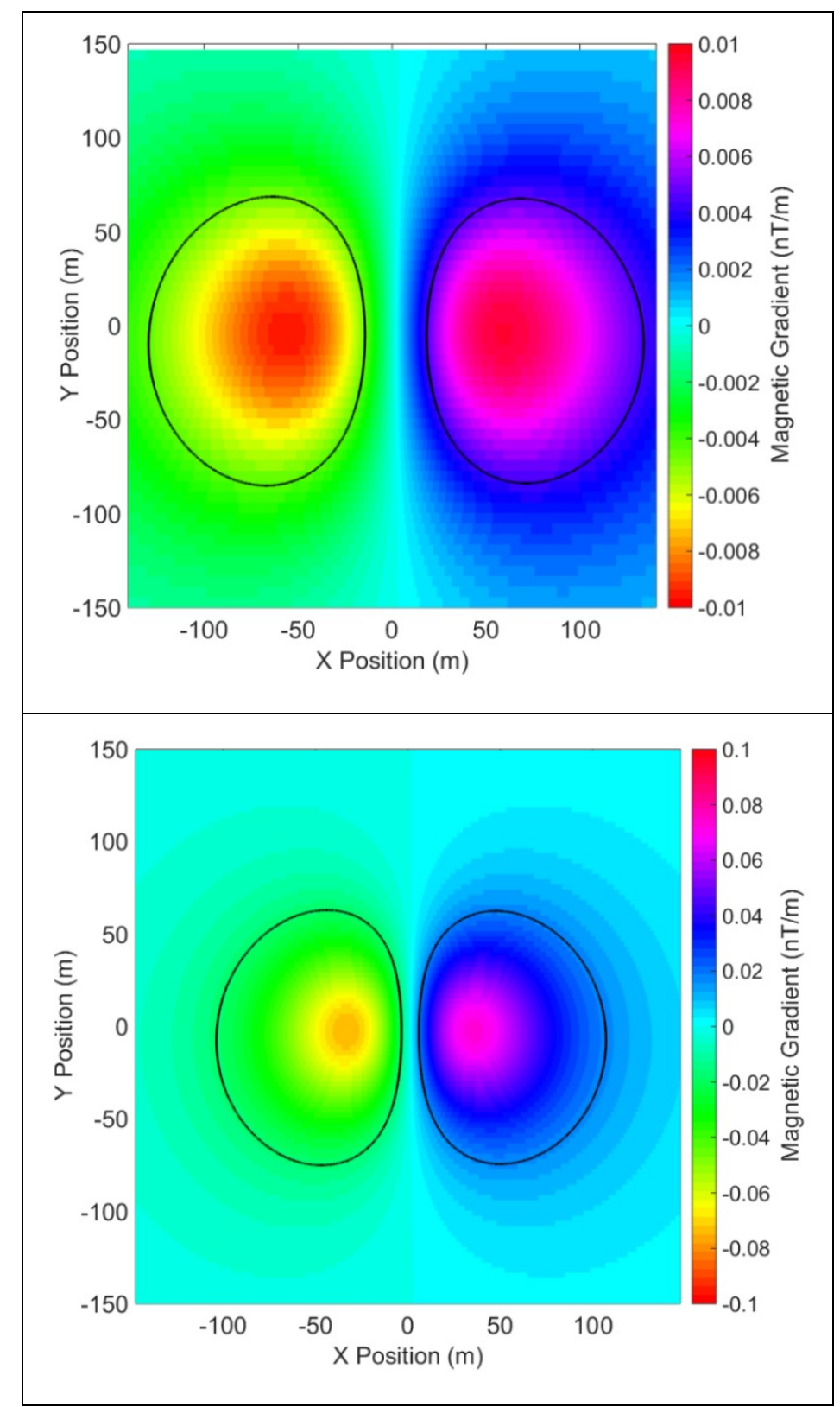

Figure 3.11: Transverse magnetic gradient map corresponding to the TMI anomaly shown in Figure 3.6 for (top) a 100m altitude AGL (traditional fixed-wing system) and (bottom) a $50 \mathrm{~m}$ altitude AGL (the Venturer UAS). The solid black contour lines encompass the detectable region. 


\subsection{3 - Spatial Resolution Limits}

Figure 3.12 presents the relationships between spatial resolution and body parameters for the three systems of interest. In the top left, the limiting depth of the two bodies is set to $10 \mathrm{~m}$ and the relation between the resolution limit and magnetic susceptibility is presented for three different body radii $(5 \mathrm{~m}, 10 \mathrm{~m}$ and $15 \mathrm{~m})$. In the top right, the radius of the two bodies is set to $10 \mathrm{~m}$ and the relation between the resolution limit and the magnetic susceptibility is presented for three different limiting depths $(10 \mathrm{~m}, 20 \mathrm{~m}$, and $30 \mathrm{~m})$. As shown in these two plots, the magnetic susceptibility does not affect the resolution limit. Because of this any magnetic susceptibility can be selected to produce the plots shown in the bottom left and right. The bottom left plot shows the relation between the resolution limit and body radius for three limiting depths $(10 \mathrm{~m}, 20 \mathrm{~m}$, and $30 \mathrm{~m})$ revealing a linear trend. As body radius increases, the signal from each body becomes stronger and broader, which causes the two anomalies to merge at shorter separation distances. The bottom right shows a limiting depth increase of approximately $10 \mathrm{~m}$, there is a resolution limit increase of approximately $5 \mathrm{~m}$ for each system. Comparing these two bottom plots shows that the resolution limit is more sensitive to the limiting depth than to the body radius.

Figure 3.13 summarizes the relation between the spatial resolution limit, the limiting depth, and radius of two neighbouring bodies for each of the three systems of interest. 


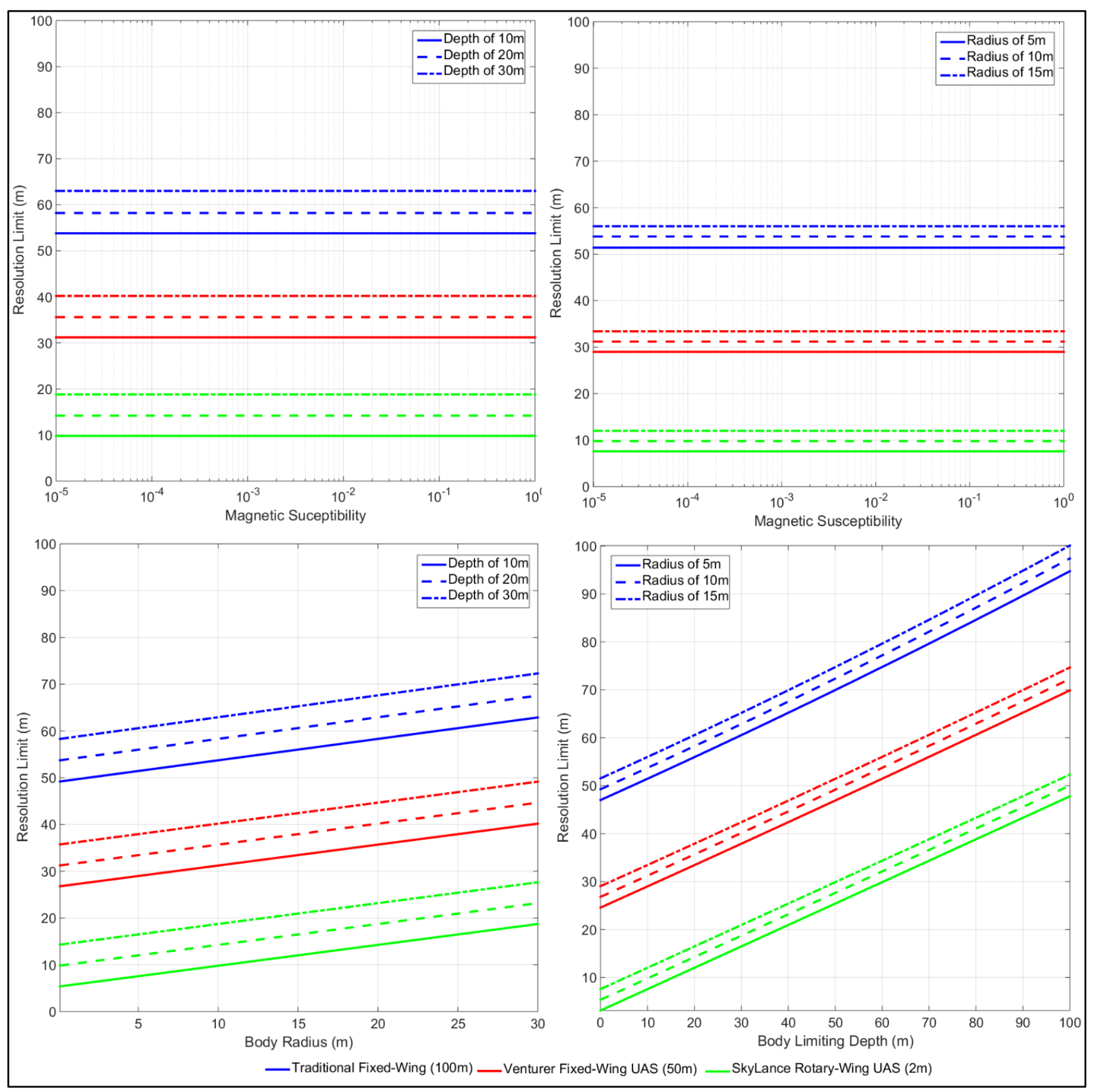

Figure 3.12: Resolution limits between two magnetic bodies for each system. The resolution limit is shown as a function of: top left - magnetic susceptibility with the limiting depth set to $10 \mathrm{~m}$, top right - magnetic susceptibility with body radius set to $10 \mathrm{~m}$, bottom left - body radius for any magnetic susceptibility, and bottom right limiting depth for any magnetic susceptibility. 


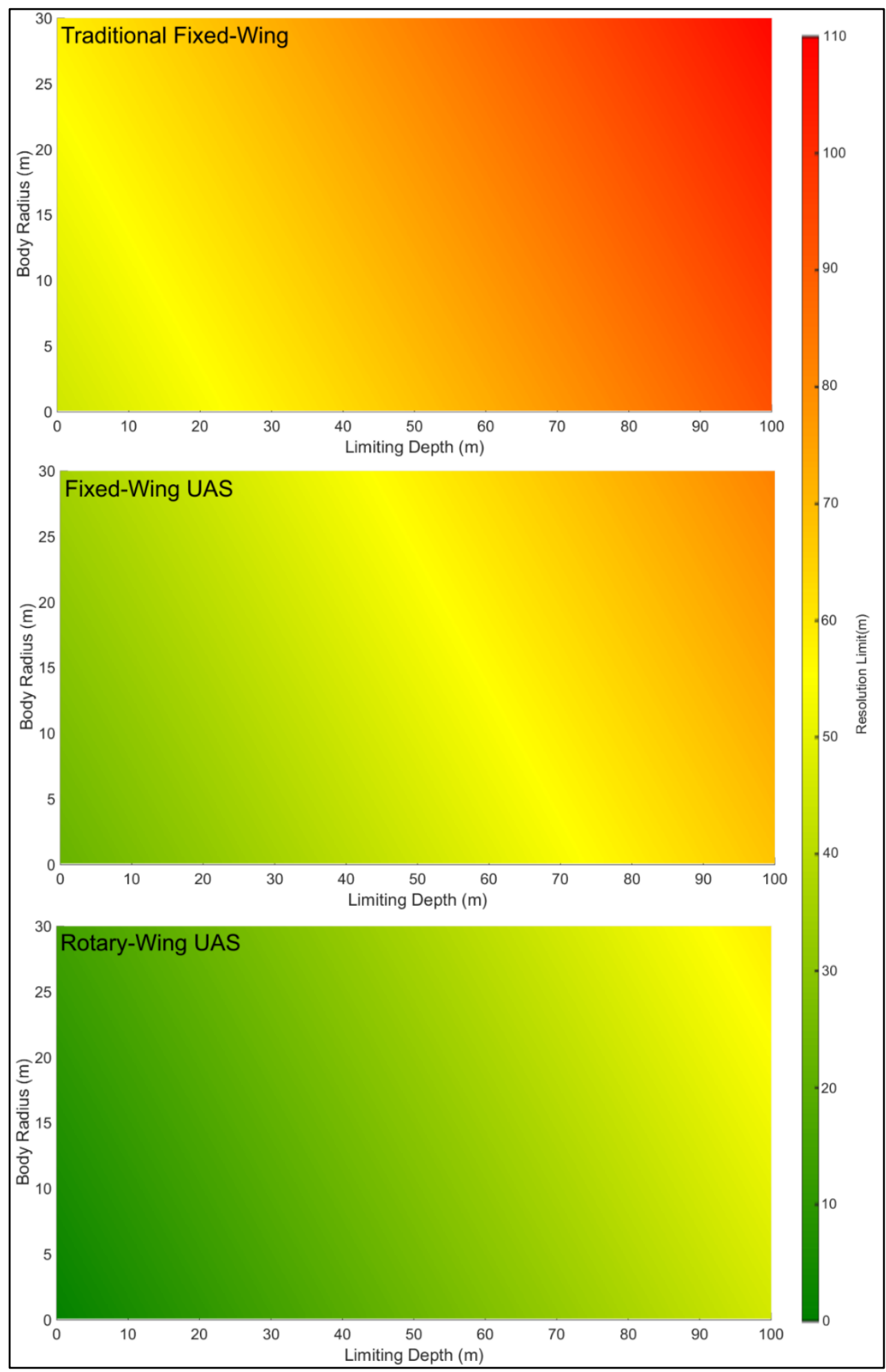

Figure 3.13: Comparison of the resolution limit for the three survey systems under consideration with respect to the limiting depth and radius of two neighbouring bodies. The traditional fixed-wing is at an altitude of 100m AGL; the fixed-wing UAS is at an altitude of $50 \mathrm{~m}$ AGL; and the rotary-wing UAS is at an altitude of $2 \mathrm{~m}$ AGL. 


\section{3 - Discussion}

When compared with traditional airborne surveys, the main advantage of UASs, as shown in the analyses presented in Chapter 3, is their lower flight altitudes. Flying closer to the ground translates to recording a fainter signal; deeper, smaller, or weakly magnetic bodies are being detected.

The first part of this analysis, determining the minimum detection capabilities, shows that at lower flight altitudes of 50m and 2m AGL, UASs are capable of detecting smaller, less magnetic, and deeper targets compared to traditional survey systems with flight altitudes of $100 \mathrm{~m}$ AGL. This is consistently shown over a range of body radii, magnetic susceptibilities, and limiting depths.

The estimates presented for minimum detection capabilities are likely a "worst case" scenario since aeromagnetic noise levels used are determined from the Venturer UAS survey (Chapter 4). This noise level is at the industry standards range of $\pm 0.05 \mathrm{nT}$. Traditional survey systems (i.e. the Cessna Grand Caravan) and further improved UASs could potentially detect even smaller body radii and smaller magnetic susceptibilities, as well as larger limiting depths due to a reduction in these noise levels.

It was also shown that fixed-wing UASs are capable of providing similar gradiometry results to what traditional systems might provide. A decrease in magnetometer spacing reduces the differences between the two magnetometers causing a decrease in gradient capabilities. This is compensated for by decreasing the aircraft flight altitude. As the aircraft flies lower, the TMI from subsurface geology is 
increased, improving gradiometry capabilities. Thus the combined decrease in magnetometer spacing and decrease in flight altitude results in little change of gradiometry capabilities.

Finally, it was shown that primarily because UASs are flying at lower altitudes, but also at slower speeds, they are capable at delineating more closely spaced magnetic targets than traditional aircrafts. Higher flight altitudes, such as the $100 \mathrm{~m}$ AGL, cause the magnetic signal from two closely space magnetic anomalies to blend together. At lower altitudes, the magnetic signal from the two anomalies does not blend together as much allowing for the identification of these two targets individually. This allows to better target future ground surveys and exploration drilling projects.

Currently, the traditional fixed-wing systems are used to survey large areas. UASs, such as the Venturer fixed-wing UAS, are a good choice for the next step in the exploration process which is to survey specific mineral exploration properties at a higher resolution. These UASs can provide detailed maps over the magnetically interesting areas. Smaller UASs, such as the SkyLance rotary-wing UAS, would be used for very small, targeted, areas which were previously identified by the larger UAS or traditional systems. Specific targets would be further resolved (for example sets of mineralized dykes would be identified as distinct anomalies) and this information used to inform drilling plans. 


\section{4 - Claresholm, AB}

On October 2, 2013 an experimental survey with the Venturer UAS was performed approximately 100km south of Calgary Alberta, Canada, near Claresholm, Alberta, over a flat plain, east of the Rocky Mountains foothills (Figure 4.1). As seen in Figure 4.2, the region is geomagnetically uniform, there is a variation of approximately 20nT within the Venturer UAS survey site. The area is underlain by approximately $1 \mathrm{~km}$ of sedimentary rocks above the Precambrian basement (Geiger, 1968). The Venturer UAS survey was conducted over a farm field, with little cultural magnetic noise from nearby structures and vehicles.

\section{1 - Experimental Survey}

On the day of the survey the weather was cool $\left(6^{\circ} \mathrm{C}\right)$ and sunny. There was little to no wind on ground level. A magnetic base station was set up to record the ambient magnetic field for post-flight diurnal corrections (Figure 4.3). Space Weather Canada reported unsettled and active solar activity for the sub-auroral zone where the survey was located which could cause high magnetic noise; however, there were no signs of this in the dataset recordings.

The total flight time, take-off to landing, was approximately 45 minutes. The UAS was tele-operated for both take-off and landing; while in flight it flew autonomously between predefined waypoints. The air speed was set to be $90 \mathrm{~km} / \mathrm{h}$ and the nominal altitude (AGL) was set to $150 \mathrm{~m}$. 
The flight path of the Venturer UAS can be seen in Figure 4.3 where the coloured sections represent the traverse and tie-lines, and the variation from the nominal altitude with each line labelled by its line number (ordered with respect to the order they were flown). At the onset of the survey, six loops were flown (shown in grey in the northeast section of Figure 4.3) to check that all systems were functioning properly. A total of seven east-west traverse lines approximately $1.5 \mathrm{~km}$ in length were then flown with $120 \mathrm{~m}$ spacing between each line; an eighth line, L1500, was ignored due to its non-straight flight path. Three north-south tie-lines approximately $0.8 \mathrm{~km}$ in length were flown perpendicular to the traverse lines and spaced $550 \mathrm{~m}$ apart. This corresponds to a total of 13 line kilometres. 


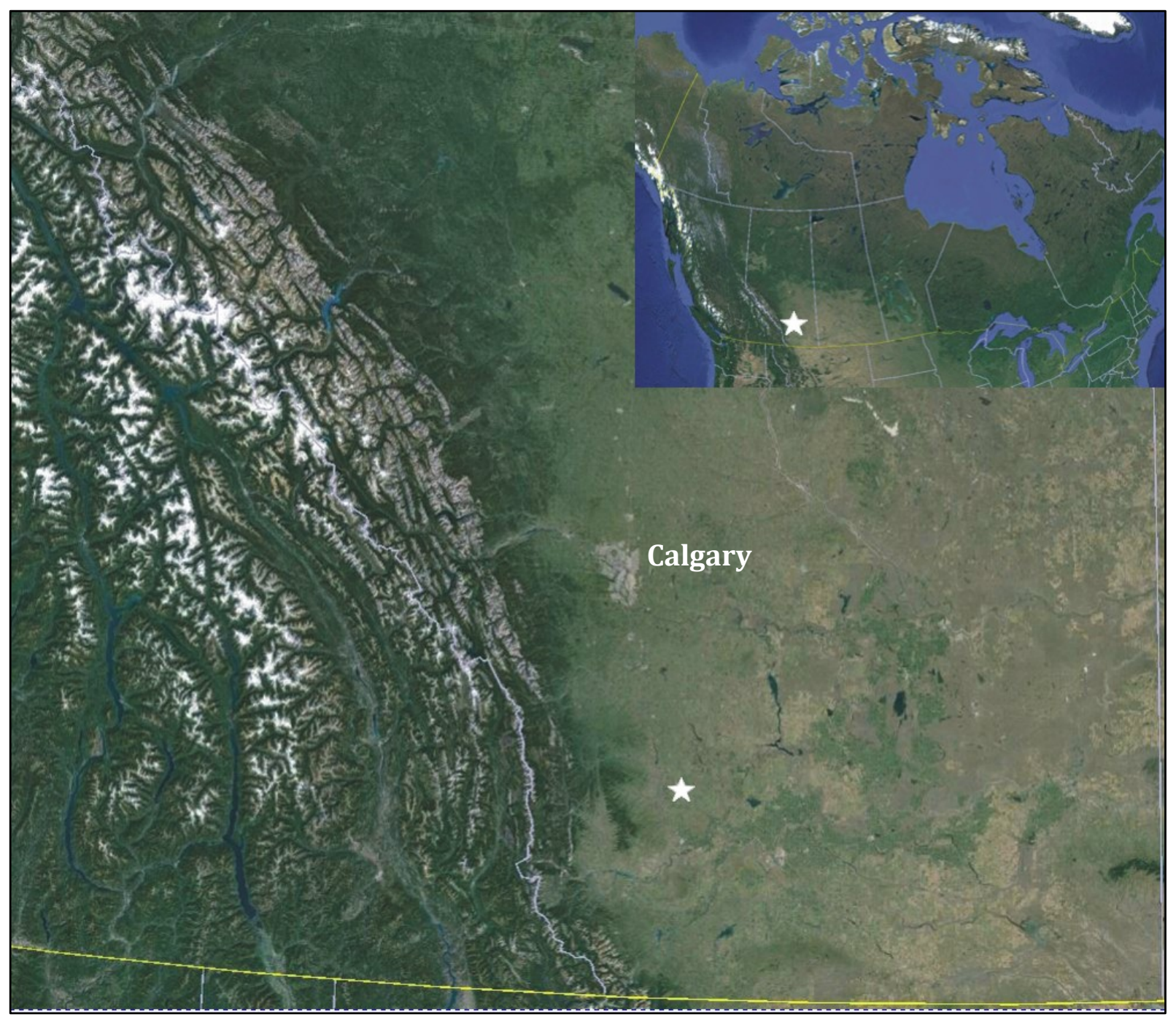

Figure 4.1: Venturer UAS survey site in southern Alberta near Claresholm; labeled with a white star. Source: Google Earth. 


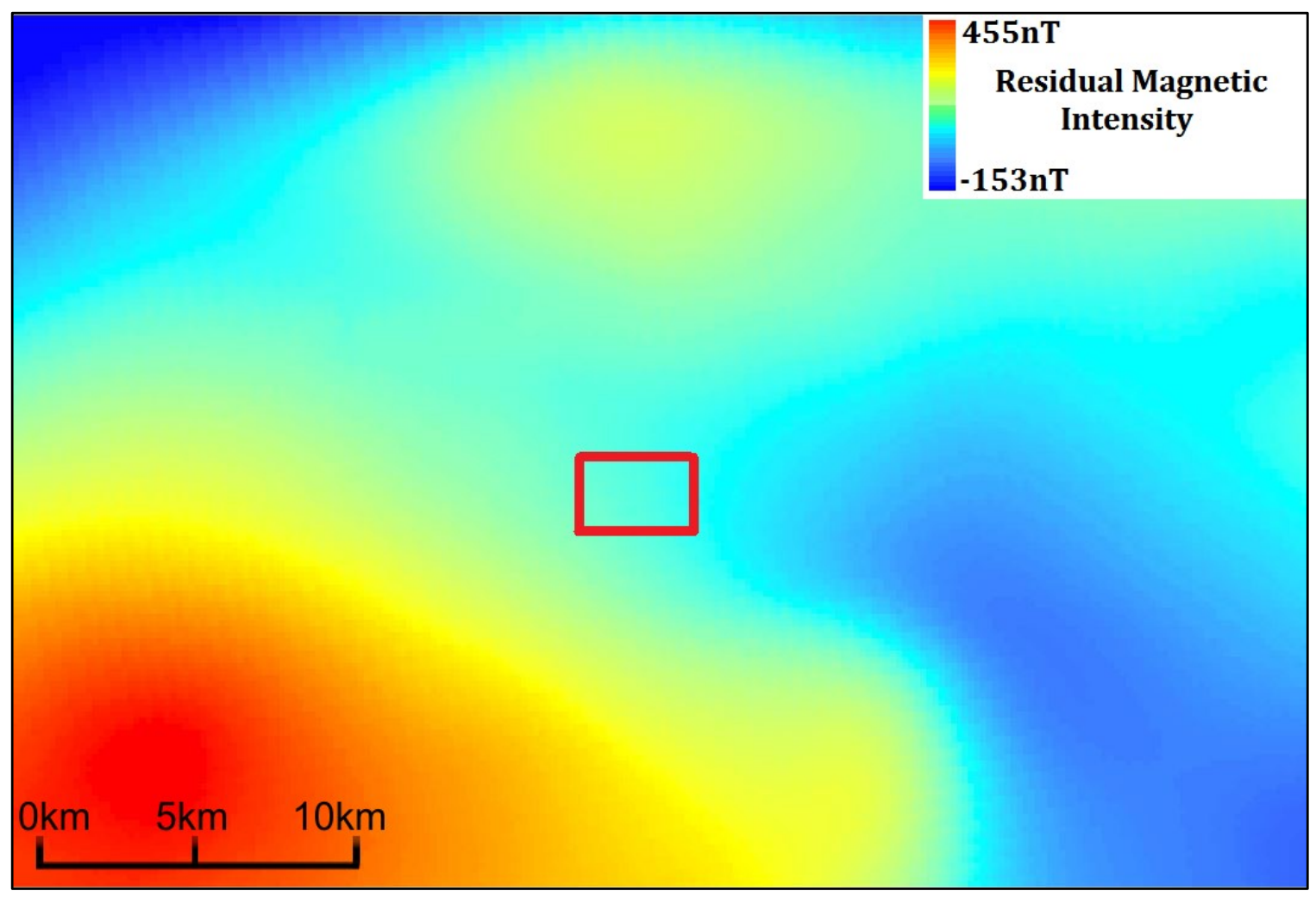

Figure 4.2: Regional residual magnetic intensity map around Claresholm, $A B$. The Venturer UAS survey site is outlined by the red box. 


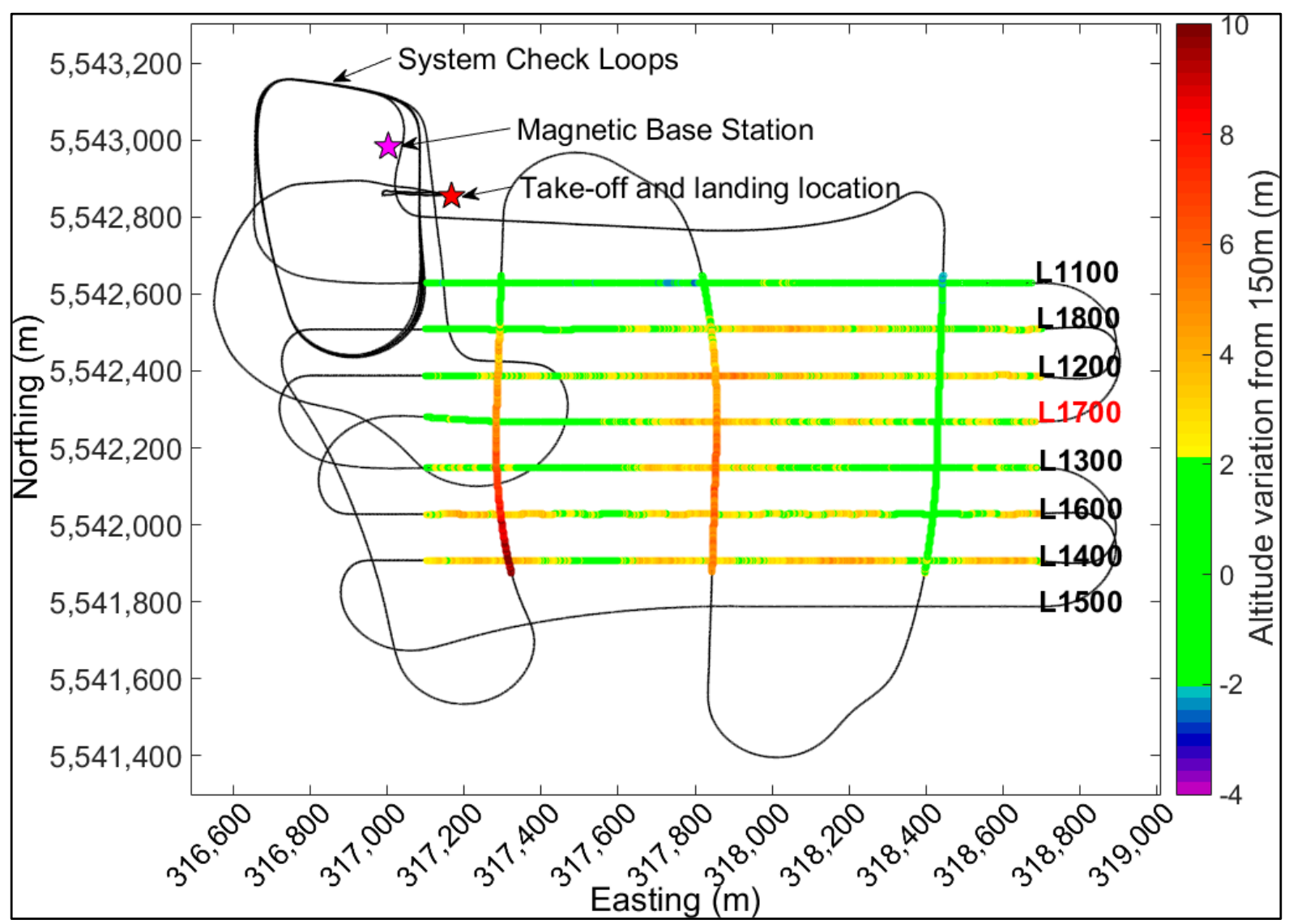

Figure 4.3: Venturer UAS flight path of the experimental survey with color-coded variations from the nominal AGL altitude $(150 \mathrm{~m})$. Flight line numbers are shown to the right of each traverse line. The profile plot of line L1700 is shown in Figure 4.3. Survey lines are in color whereas turns and system check loops are in black. 


\section{2 - Data Processing}

Traditional surveys require (depending on contract specification) that altitude variations are within $\pm 20 \mathrm{~m}$ and aircraft pitch and roll variations being within $\pm 10^{\circ}$ and $\pm 5^{\circ}$, respectively. In comparison to this, the Venturer UAS proved to be very stable in flight. Along the traverse and tie-lines, the average altitude AGL was $152.1 \pm 1.4 \mathrm{~m}$ and the average flight speed (with respect to the ground) was approximately $90 \pm 22 \mathrm{~km} / \mathrm{h}$. The large standard deviation attached to flight speed is due to wind effects. As shown in Figure 4.3, variations from the nominal AGL altitude were within a few meters. The largest variations mostly coincide with the ends of line and are associated with turning manoeuvres commanded by the autopilot system. Along individual flight lines, the standard deviation associated with pitch and roll angles were on the order of $\pm 0.7^{\circ}$ and $\pm 3^{\circ}$, respectively.

One of the two magnetometers did not provide quality data due to an internal fault with the unit; however, the second magnetometer performed well. Since the UAS demonstrated good flight stability and because its airframe is composed of nonmagnetic material, no heading or attitude corrections were applied to the total magnetic intensity (TMI) data acquired by this magnetometer. The data presented here has not been compensated by the use of hardware or software. Compensation of aeromagnetic data acquired using UASs remains an open research question.

In Figure 4.4, the raw flight data of line L1700 (see Figure 4.3) is provided as well as the post-flight processing results. Post-processing included a diurnal 
correction and a simple tie-line levelling correction based on a $1^{\text {st }}$ polynomial fit of the 3 tie-line crossover points after the application of the diurnal correction. 


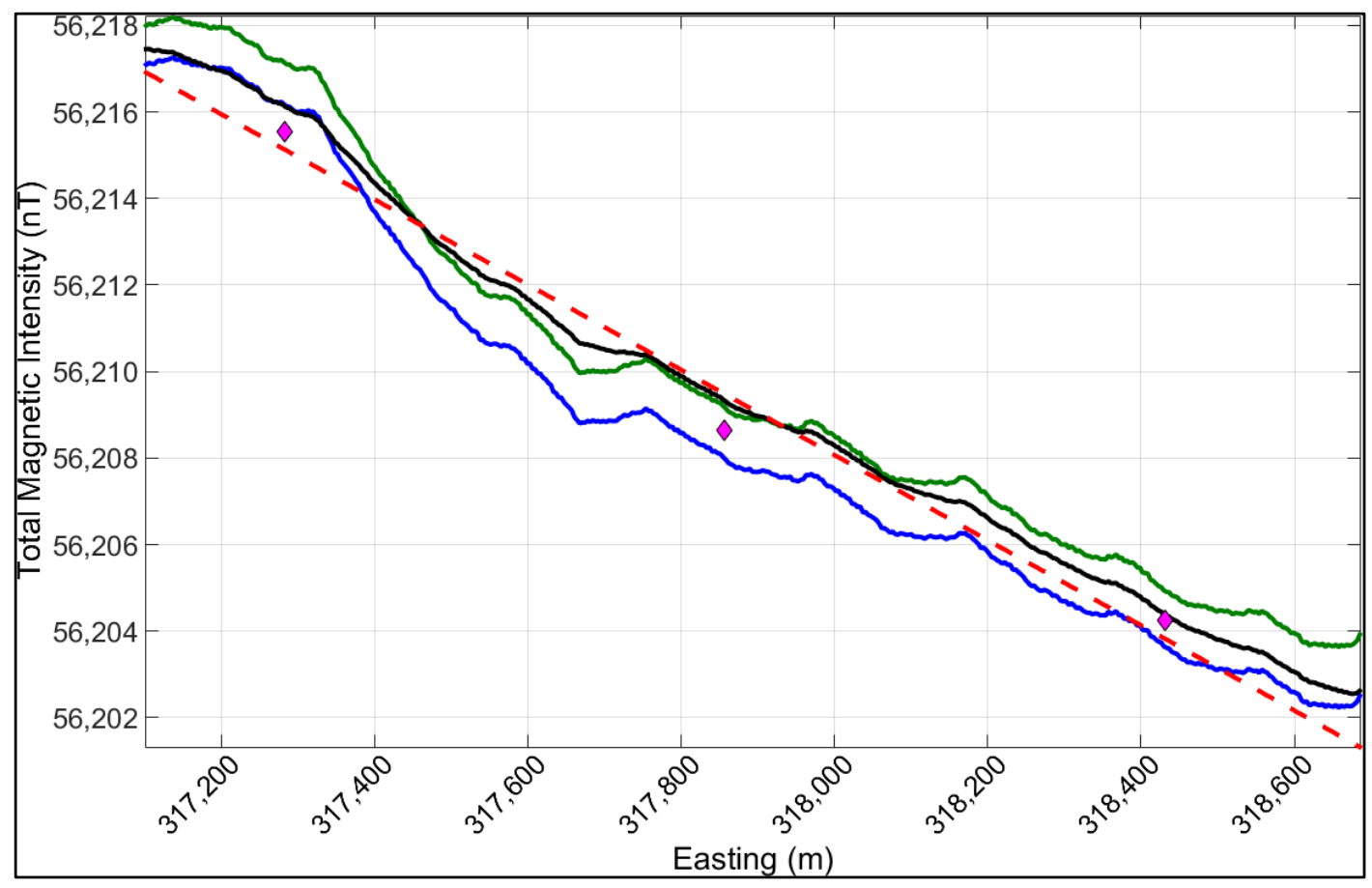

Figure 4.4: Total magnetic intensity (TMI) along line L1700. The raw data is in blue. The data corrected for diurnal variations is in green. The tie-line levelled data is in black. The tie-line crossover points are in magenta and the $1^{\text {st }}$ order polynomial fit used for tie-line levelling is in red. 
To determine the noise levels on the survey data, the fourth difference was calculated (Teskey et al., 1991; Coyle et al., 2014). Figure 4.5 shows the fourth difference noise levels for the magnetic data from all the traverse and tie-lines (6453 data points included in the analysis). One standard deviation from the mean is equal to $\pm 0.014 \mathrm{nT}$. The noise level range is approximately $\pm 0.05 \mathrm{nT}$ ( $0.1 \mathrm{nT}$ peak-to-peak); this is at the industry accepted noise level of $\pm 0.05 \mathrm{nT}$ (Teskey et al., 1991; Coyle et al., 2014).

Because the UAS is set to fly nominally at $90 \mathrm{~km} / \mathrm{h}$ airspeed, wind can have an effect on the UAS speed with respect to the ground. The flight path of the UAS along the traverse lines is shown in Figure 4.6, where each black dot represents every tenth magnetic intensity reading, which corresponds to a sampling rate of $1 \mathrm{~Hz}$. From this display, it is possible to visualize the effect of wind on the UAS. Although ground conditions were not windy, it is likely that there was an eastward wind at flight altitude. Due to this eastward wind, the UAS flew faster when travelling east $(124 \pm 2 \mathrm{~km} / \mathrm{h})$ than when travelling west $(70 \pm 2 \mathrm{~km} / \mathrm{h})$. The wind, however, was not strong enough to cause significant deviation from the desired flight paths. When fitting a straight line to flight segment, the average standard deviation is $0.9 \mathrm{~m}$ for traverse lines and $25 \mathrm{~m}$ for tie-lines. There is a larger variation in the tie-lines due to the flight plan given to the autopilot; some turns were sharp and close to the boundaries of the survey area, which did not leave enough time for the aircraft to be perfectly straight at the beginning of a survey line. In addition, the wind was blowing to the east which affected the north-south oriented tie-lines more than the east-west oriented traverse lines. 


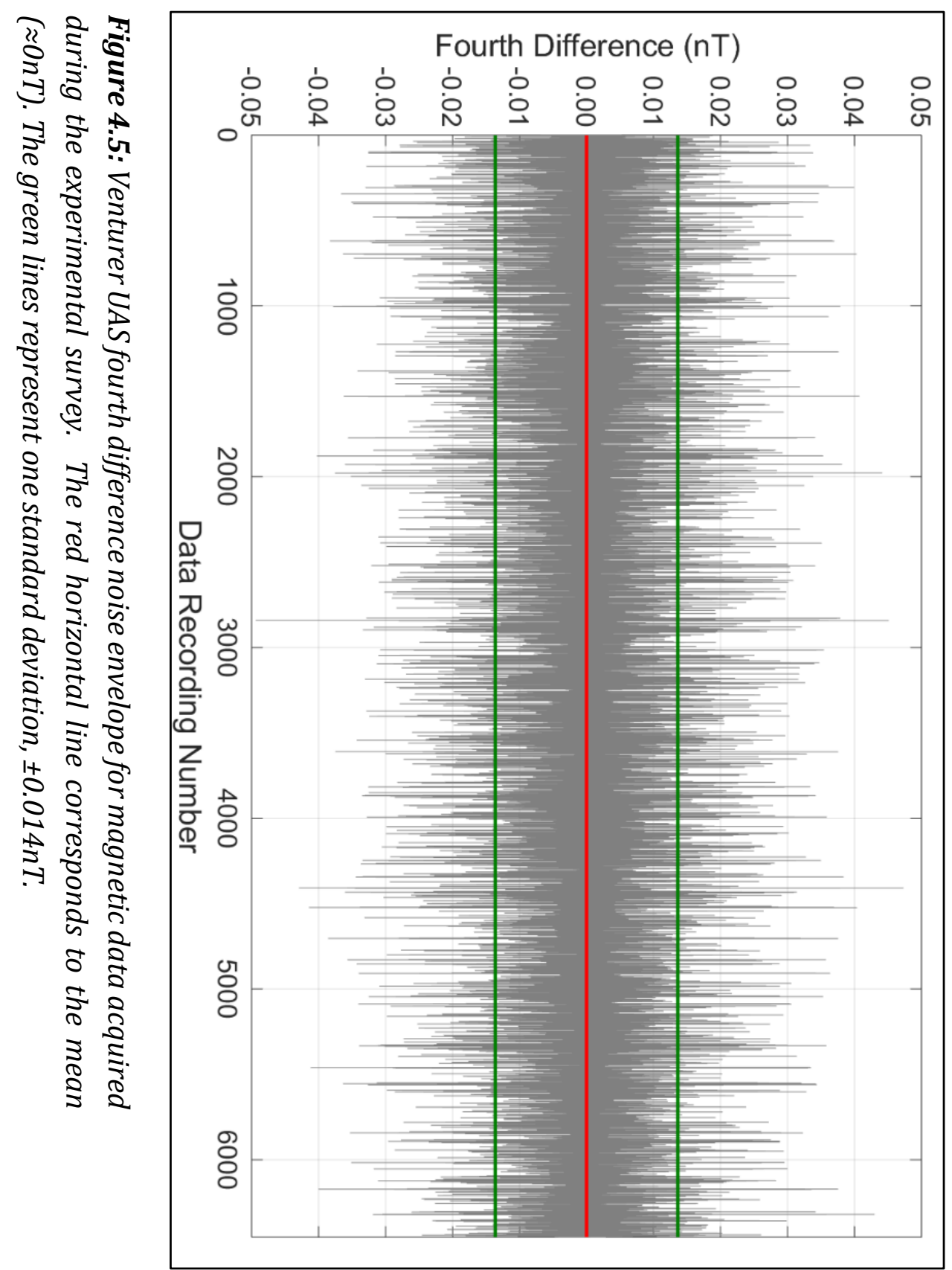




\section{3 - Discussion}

Following the application of the diurnal and tie-line leveling corrections to the survey data, a minimum curvature interpolation at $1 / 4$ of the flight line spacing was performed to produce the TMI map (Lee and Morris, 2013). This detailed map (Figure 4.6) shows a gradual increase of approximately $18 \mathrm{nT}$ from east to west, similar to the 20nT variation shown in Figure 4.2, which is interpreted to correspond to a decrease, from east to west, in sediment thickness in the area.

This experimental survey demonstrated that the Venturer UAS is a very stable platform in flight. Depending on contract specifications, tradition surveys require altitude variations to be between $\pm 20 \mathrm{~m}$ as well as pitch and roll variations to be within $\pm 10^{\circ}$ and $\pm 5^{\circ}$. The Venturer had a maximum variation from $150 \mathrm{~m}$ altitude AGL of only $12 \mathrm{~m}$ and a pitch and roll variation of $\pm 1.5^{\circ}$ and $\pm 4^{\circ}$, respectively. The UAS only required operator intervention during take-off and landing. It required no intervention during flight. The care in reducing to a minimum the magnetic components in the airframe, the high accuracy in positioning provided by differential GPS, and the excellent aircraft manoeuvrability all contributed to the acquisition of high-quality magnetic data with a noise envelope $( \pm 0.05 \mathrm{nT})$ that is within the acceptable range for conventional systems.

There was a $+2 \mathrm{~m}$ offset from the nominal AGL altitude of $150 \mathrm{~m}$ with a standard deviation of $\pm 1.4 \mathrm{~m}$. The deviation from a straight flight path was approximately $\pm 0.9 \mathrm{~m}$ along traverse lines and $\pm 25 \mathrm{~m}$ along tie-lines. These larger tie-line variations were primarily due to the UAS turning manoeuvres and to cross wind effects. When aiming for a specific flight line, it handled approach and turns well except for the southernmost 
flight line (L1500). In future surveys, it will be important to extend the end of line waypoints far enough out that the turning manoeuvres of the UAS do not occur above the area of interest. This is critical when the separation between survey lines is reduced and the autopilot must make adjustments due to tight turning radii. 


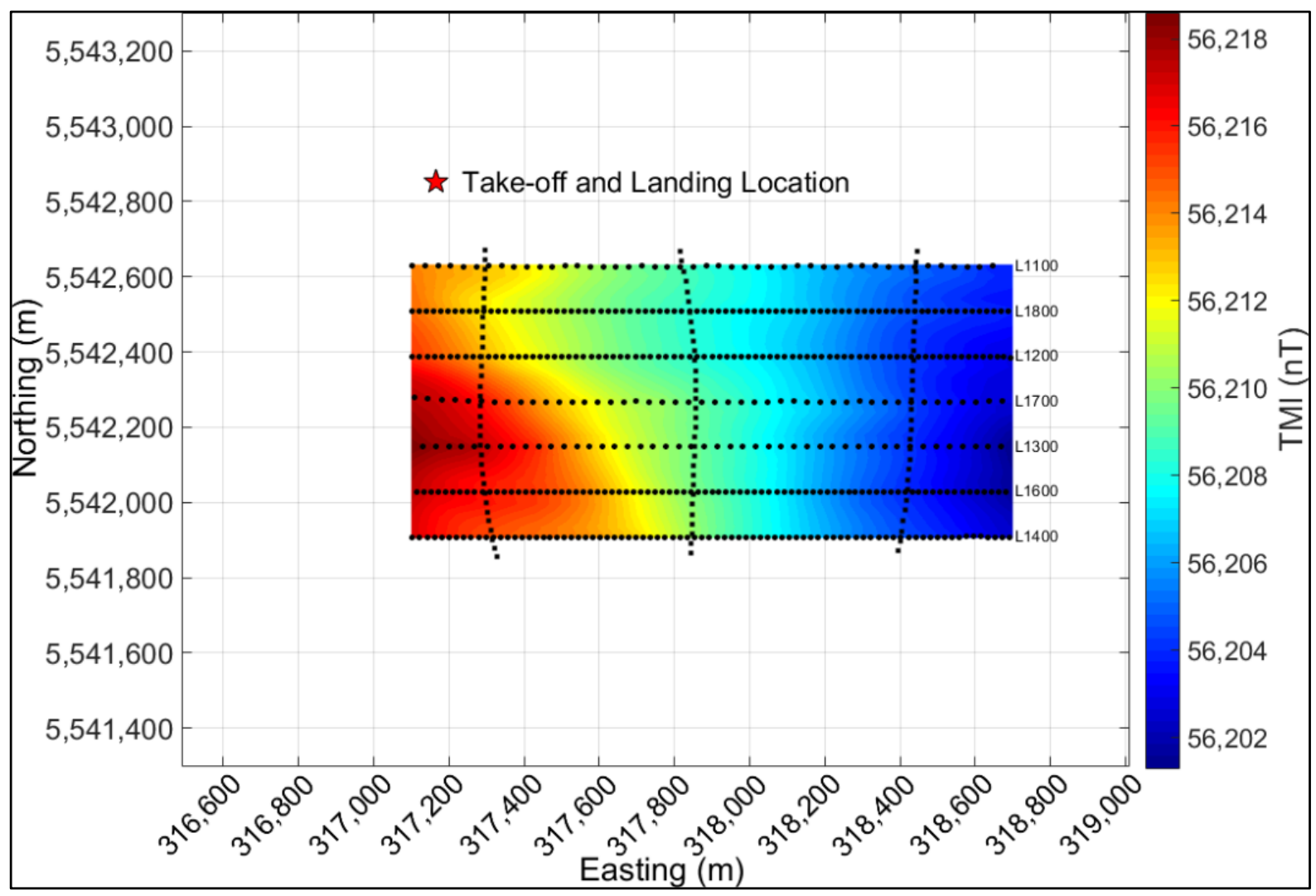

Figure 4.6: Venturer UAS TMI map of the experimental survey area, with diurnal and tie-line leveling corrections applied together with a minimum curvature interpolation. Flight line numbers are shown to the right of each traverse line. 


\section{5 - Nash Creek, NB}

In August and October, 2015, co-located ground magnetic and aeromagnetic surveys were performed just outside of Nash Creek, New Brunswick over a known magnetic anomaly. The Nash Creek property, highlighted by a red star in Figure 5.1, is owned by Slam Exploration Ltd. and is located just off the southern coast of Chaleur Bay in New Brunswick, and south of the Gaspé Peninsula, Québec. The property is between two northeast trending orogenic belts; the Aroostook-Percé (north) highlands and the Miramachi highlands (south) (Bongajum, 2011). The property is also situated approximately 50km northwest of the Bathurst Mining Camp (BMC) which is located in the Miramachi highlands.

The BMC has many occurrences of volcanic massive sulfides which were deposited within Cambro-Ordovician rocks. The Nash Creek property, however, sits on younger rocks of Lower Devonian age (Bongajum, 2011). The rocks in Nash Creek are composed of volcanic breccias, siltstones, limestones, mafic flows, rhyolites, and tuffs where the main source of mineralization is within the Dalhousie group. Brown (2007) suggests that the mineralization occurred within a failed rift system filled with shallow water. Volcanic and sedimentary rocks were deposited in a half-graben where a fault boundary allowed for the flow of hydrothermal fluid causing sulfide mineral accumulation containing Zinc ( $\mathrm{Zn})$, Lead (Pb), and Silver (Ag).

The Nash Creek property contains no known outcrops, so it is a "blind" deposit. It represents a unique style of mineral deposit as it does not feature the typical characteristics of sedimentary extrusive (SEDEX), volcanic massive sulphide (VMS) 
or Missippi-Valley Type (MVT) deposits (Milkereit, personal communication, 2015). It is suggested that the deposit is related to a deep intrusive body. The rocks of the Nash Creek property were affected by the hydrothermal fluids which were regulating the temperature of this deep body.

Extensive exploration, primarily drilling, has been performed on the Nash Creek property for approximately 60 years (Ugalde, et al., 2007). Various geophysical surveys have also been performed, including an aeromagnetic survey resulting in the map shown in Figure 5.2. The black path represents a ground magnetic survey performed by Prof. Bernd Milkereit and his students from the University of Toronto in 2014. Two magnetic anomalies were identified from this ground magnetic data (crossed hammers symbol) for future surveying.

In the rest of this section two magnetic surveys at the Nash Creek property will be discussed; a ground magnetic survey and an aeromagnetic survey flown with the SkyLance UAS both done as part of this project. Each survey will be described in Section 5.1. Data processing is presented in Section 5.2 with results and discussion in Section 5.3 and Section 5.4, respectively. 


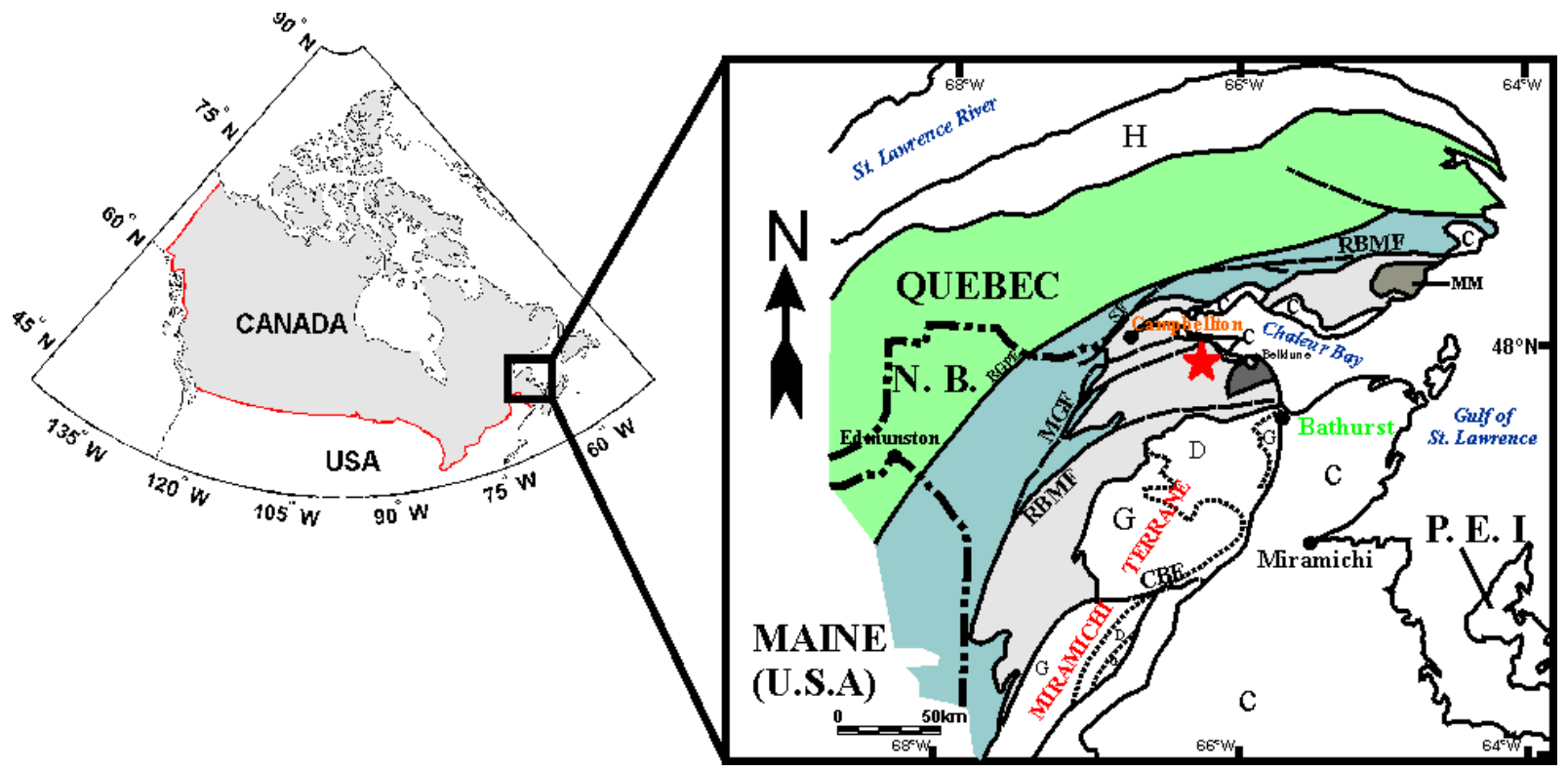

Figure 5.1 SkyLance UAS survey area (Nash Creek, New Brunswick) marked with a red star with regional geology. Green - Connecticut Valle-Gaspe Synclinorium; Blue Aroostook-Percé Anticlinorium; Light gray - Chaleur Bay Synclinorium; MGF McKenzie Gulch Fault; SF - Sellarsville Fault; RBMF - rocky Brook-Millstream Fault; CBF - Catamaran Brook Fault; RGPF - Restigouche-Grand Pabos Fault. Pre- to Late Ordovician inliers in the Gaspé Belt are shown. Dark grey - MM - Macquereau-Mictaw Inlier (Humber-Dunnage); E - Elmtree Inlier (Dunnage). From Bongajum (2011). 


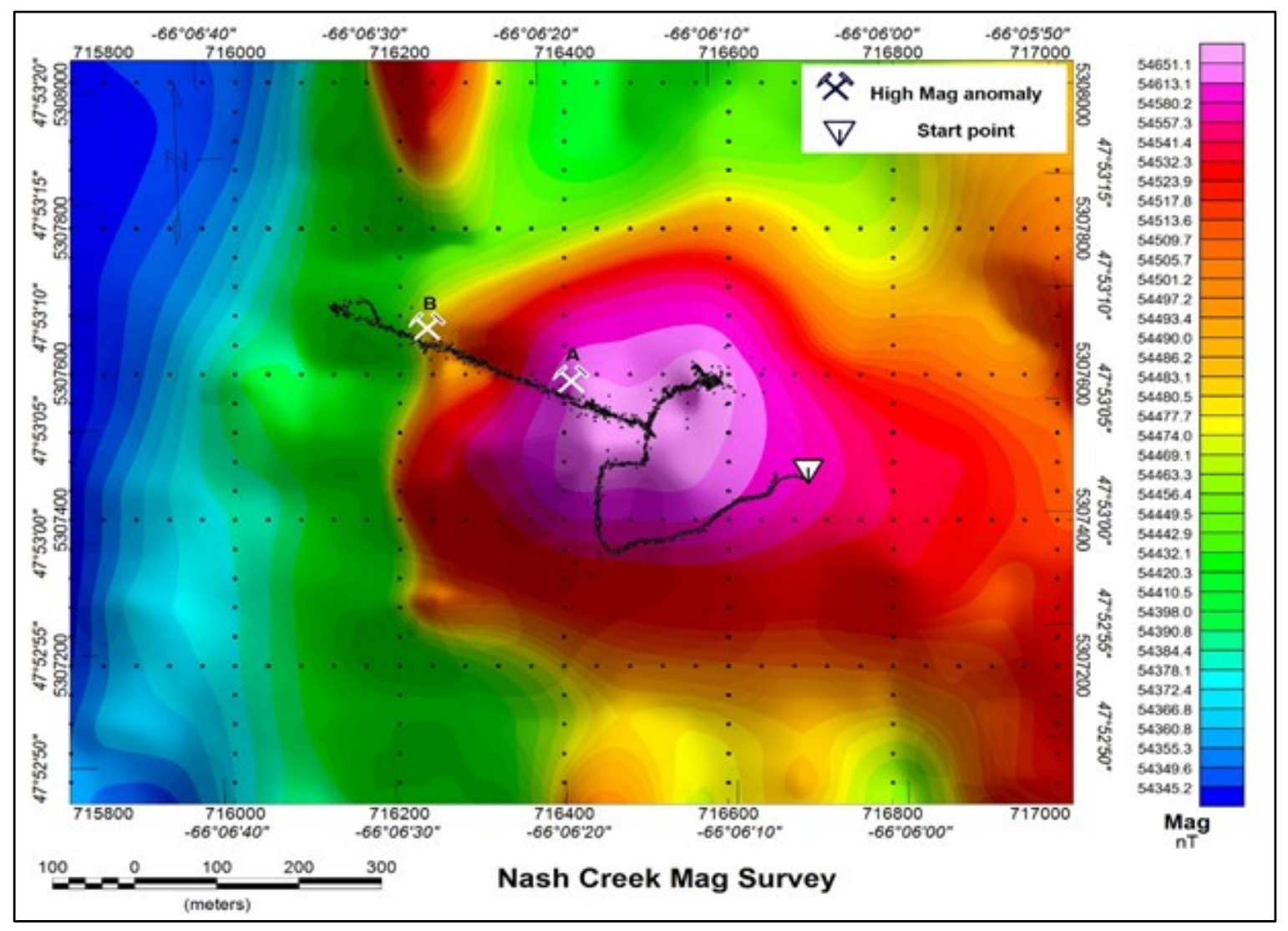

Figure 5.2: Aeromagnetic TMI survey over the Nash Creek, NB survey area. The black path represents a ground magnetic survey performed in 2014 by a team from the University of Toronto. Two magnetic highs identified on the 2014 ground data are labeled with crossed hammers. 


\section{1 - Experimental Surveys}

Two magnetic surveys were completed on the Nash Creek, New Brunswick mineral exploration property as part of this project. The first was a ground magnetic survey completed in August, 2015, and the second was an aeromagnetic survey performed by the SkyLance UAS completed in October, 2015. The primary purpose for surveying at Nash Creek, NB was to test the SkyLance UAS and to gather data for comparisons between ground magnetic and aeromagnetic surveys. Since the property has only been surveyed partially by a ground crew, an extensive ground magnetic survey was performed. The previously collected aeromagnetic data is presented in Figure 5.2.

In the following two subsections, a description of the 2015 ground magnetic and aeromagnetic surveys is presented. Data processing of the two magnetic surveys is shown in Section 5.2 .

\subsection{1 - Ground Magnetic Survey}

On August 11-13, 2015, the ground magnetic survey was executed on the Nash Creek property. The survey was performed by Andrew Hay, a fellow student doing a M.Sc. in Earth Sciences at Carleton University and myself (Figure 5.3).

The Nash Creek ground magnetic survey area is outlined in Figure 5.4 by a red box. The known magnetic anomalies are located within this outlined area. Along the western and northern edges of the box is steep topography, with a small river, flowing 
north east and then east at the bottom of a valley. Along the eastern edge of the box is a small stream flowing north. There is also a small lake, Hayes Lake at the south edge of the box. Along the eastern edge of the survey area there are also dirt and gravel access roads. As seen in the satellite image (Figure 5.4), there is a lot of vegetation (deciduous and coniferous trees). There are many areas across the property that contain dense brush and tree canopy as well as steep slopes, making it difficult to traverse (Figure 5.3).

The planned ground magnetic survey lines are shown in Figure 5.4. The ground magnetic lines are oriented east-west according to UTM easting/northing, perpendicular to the strike of the geological structures of interest. The survey lines 1150 to 1300 are approximately $850 \mathrm{~m}$ in length and lines 1350 to 1600 are approximately $1,150 \mathrm{~m}$ in length. Each line is spaced $50 \mathrm{~m}$ apart from the next. An example elevation profile is shown in Figure 5.5, along line 1550; there is a maximum elevation of $117 \mathrm{~m}$ along the western ridge and a minimum elevation of $80 \mathrm{~m}$ along the eastern side, associated with the small stream. 

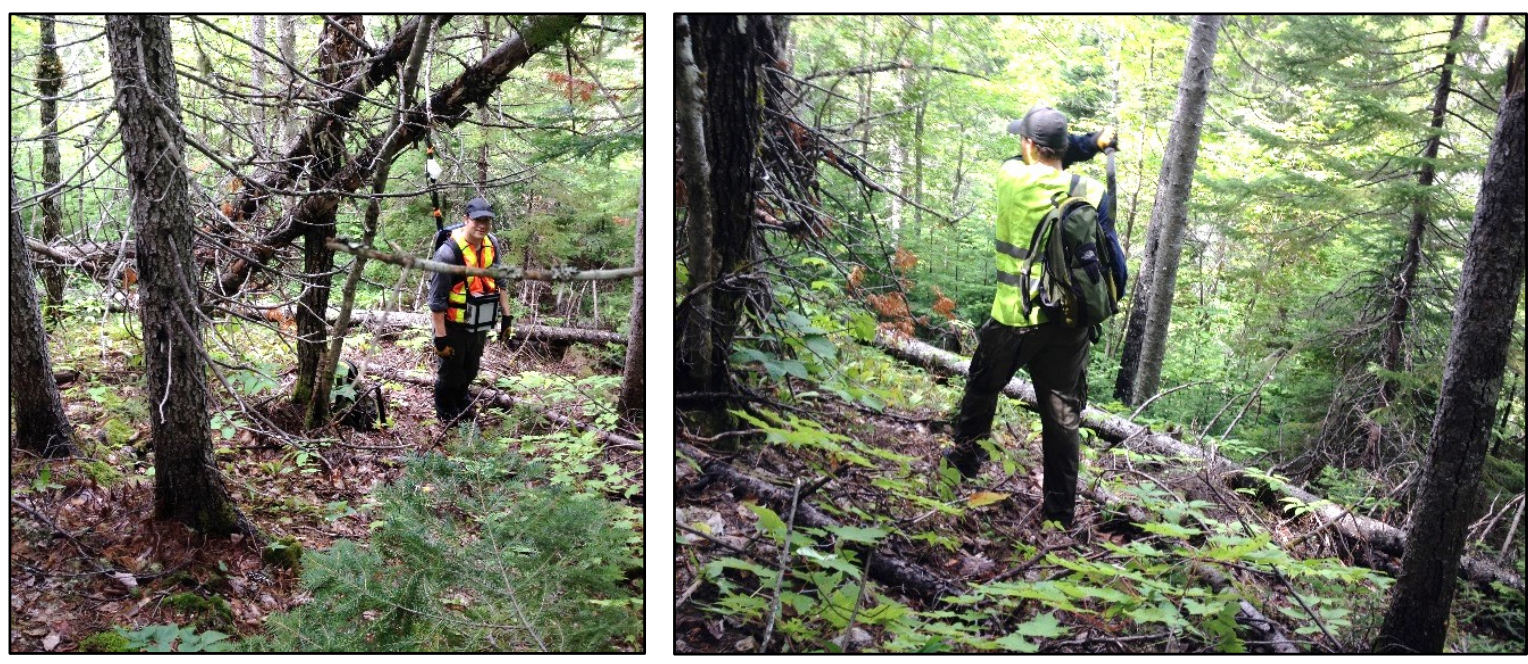

Figure 5.3: Nash Creek survey area terrain and bush. Michael Cunningham (left) and Andrew Hay (right) acquiring ground magnetic data on the property. These images show some of the terrain and dense bush encountered throughout the survey area (note that the survey also covered a few more open areas). 


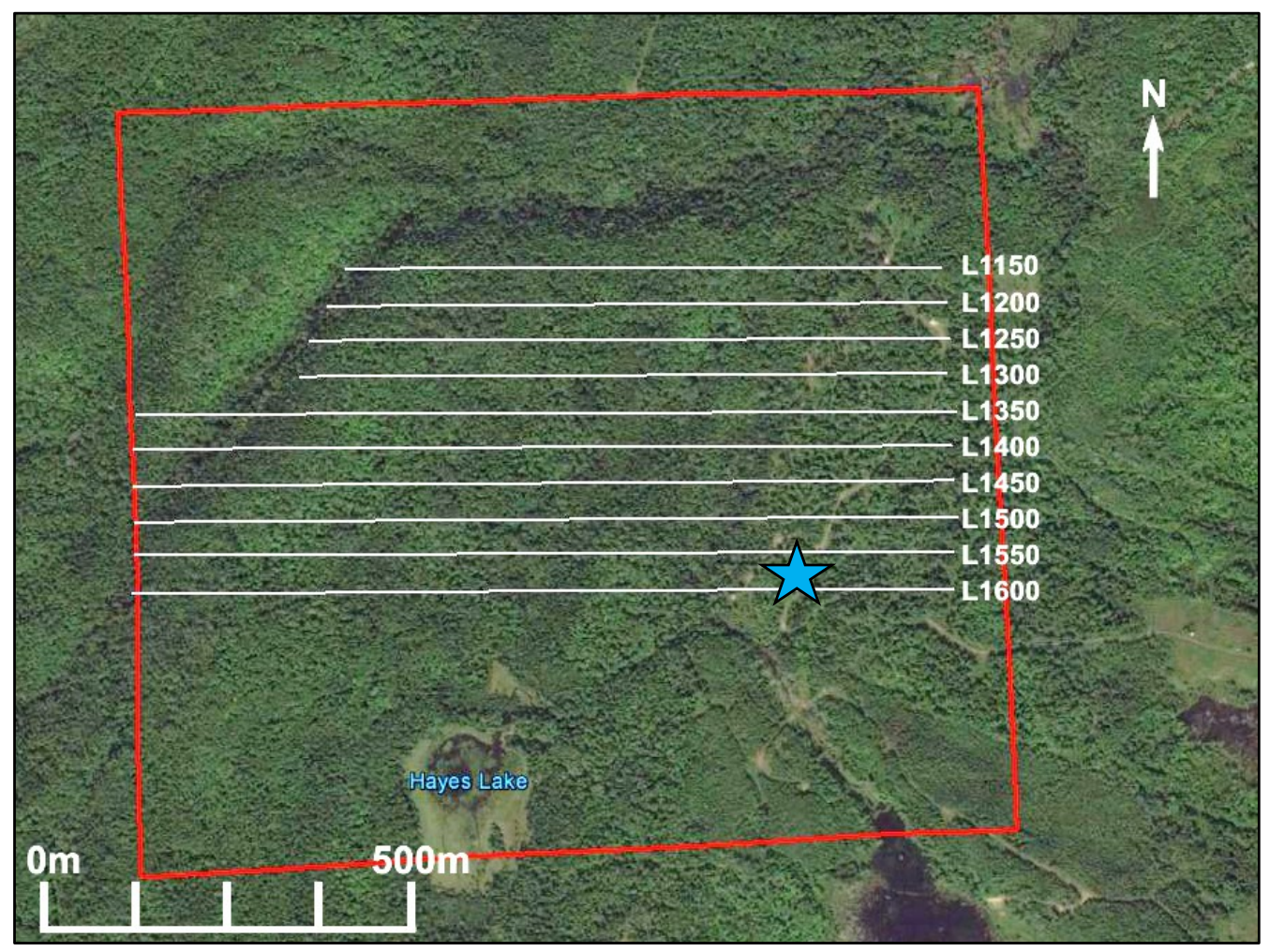

Figure 5.4: Satellite image of the Nash Creek survey area, outlined by the red box. This area coincides with the area shown in Figure 5.2. The location of this survey area is presented in Figure 5.1. Planned survey lines are labeled and drawn in white. The magnetic base station location is shown by the blue star. Image source: Google Earth. 


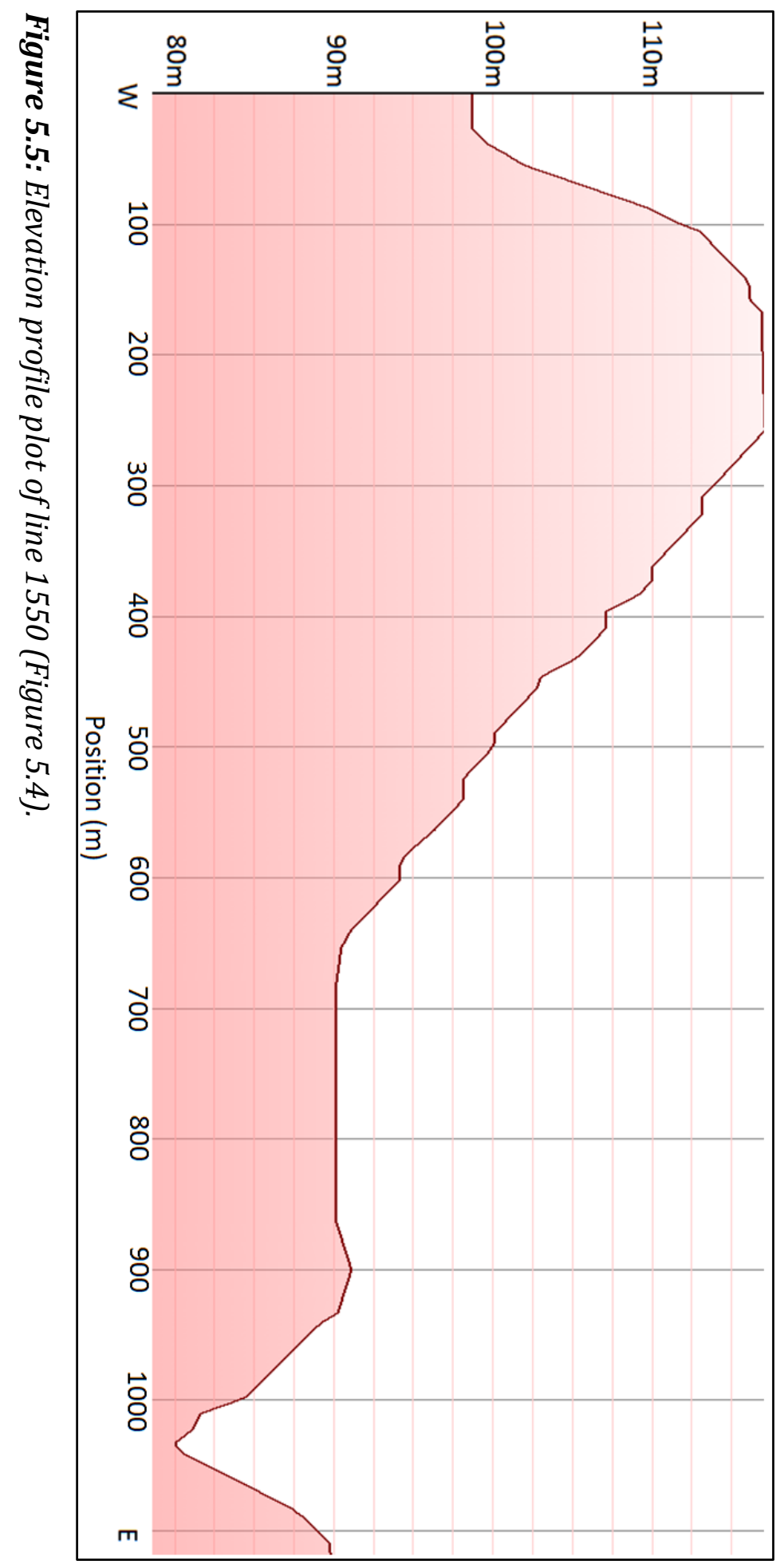


In Table 5.1 notes for each day of surveying are provided. All the planned survey lines were completed. The raw ground magnetic survey data is presented in Figure 5.6. The main issue with the raw magnetic data is the loss of GPS signal, which creates pockets of non-georeferenced data. This is primarily associated with the dense brush and canopy, as well as with the poor weather conditions (cloudy and raining). When the operator noticed that GPS signal was lost while magnetic data was still being recorded, an attempt was made to walk at a constant pace and in as straight of a path as possible in order to allow for correction of the data in post-processing.

The data processing of this magnetic survey is presented in Section 5.2.1. 
Table 5.1: Daily notes for ground magnetic survey

\begin{tabular}{|c|c|c|c|}
\hline Date: & August 11, 2015 & August 12, 2015 & August 13, 2015 \\
\hline Weather: & $\begin{array}{l}\text { Sunny morning } \\
\text { Heavy rain in } \\
\text { the afternoon }\end{array}$ & $\begin{array}{l}\text { Rain in the } \\
\text { morning } \\
\text { Periods of rain in } \\
\text { the afternoon }\end{array}$ & $\begin{array}{l}\text { Sunny with } \\
\text { clouds }\end{array}$ \\
\hline $\begin{array}{l}\text { Space } \\
\text { Weather: }\end{array}$ & Quiet & Quiet & Quiet \\
\hline $\begin{array}{l}\text { Lines } \\
\text { Surveyed: }\end{array}$ & $\begin{array}{l}\text { L1500, a third } \\
\text { of L1450, a half } \\
\text { of L1400, } \\
\text { roadways }\end{array}$ & $\begin{array}{l}\text { L1550, L1600, } \\
\text { L1350, and } \\
\text { L1400 }\end{array}$ & $\begin{array}{l}\text { L1250, L1300, } \\
\text { L1200, L1150, } \\
\text { L1450 }\end{array}$ \\
\hline $\begin{array}{l}\text { Line } \\
\text { Distance } \\
(\mathbf{k m}):\end{array}$ & Approx. 3km & Approx. $4 \mathrm{~km}$ & Approx. 5km \\
\hline Notes: & $\begin{array}{l}\text { Part way on } \\
\text { L1450 lost } \\
\text { system power. } \\
\text { Poor GPS along } \\
\text { L1400, instead } \\
\text { walked access } \\
\text { roads }\end{array}$ & $\begin{array}{l}\text { Had intermittent } \\
\text { GPS, creates } \\
\text { dead zones in } \\
\text { survey. Re- } \\
\text { walked } \\
\text { incomplete } \\
\text { L1400. }\end{array}$ & $\begin{array}{l}\text { Poor GPS along } \\
\text { northern lines. } \\
\text { Re-walked } \\
\text { incomplete } \\
\text { L1450. }\end{array}$ \\
\hline
\end{tabular}




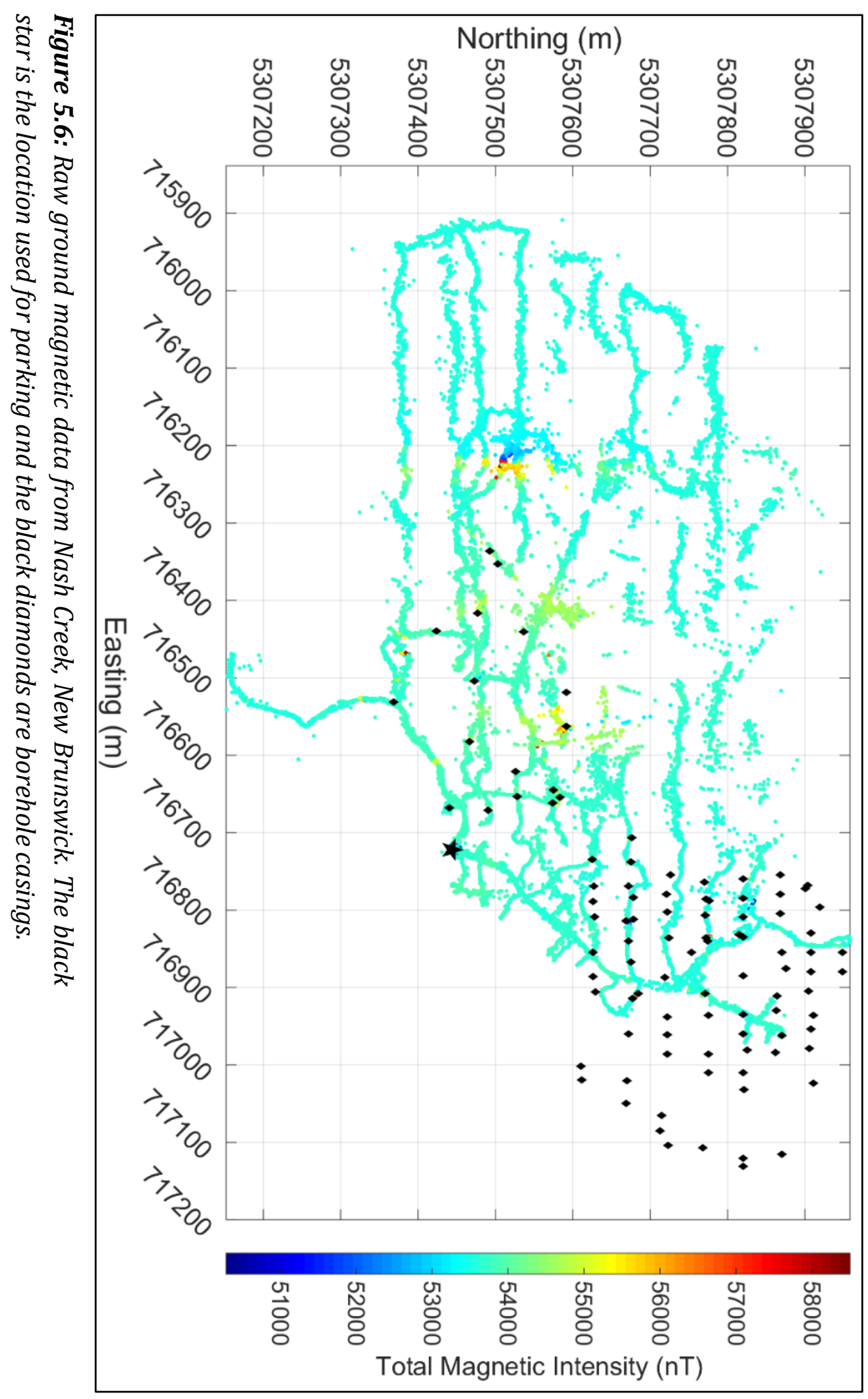




\subsection{2 - Aeromagnetic Survey}

On October 17, 2015 an aeromagnetic survey was performed with the SkyLance rotary-wing UAS at the Nash Creek property. This survey was performed by two employees from the industry partner, Stratus Aeronautics, Alan Wood and Ian Cooke, and assisted by my supervisor, Prof. Claire Samson, and myself (Figure 5.7).

The planned survey lines were to match, but extended in length on either end, those that were walked in August, 2015 (see Section 5.1.1 and Figure 5.4). From the ground magnetic survey data, lines 1450 and 1550 (as shown in Figure 5.6) were identified as the top priority for the aeromagnetic survey since they cross three strong magnetic anomalies identified in the ground magnetic data.

Prior to flying the SkyLance UAS, a smaller commercial UAS (IRIS+ by 3DRobotics Inc.) with a GoPro video camera attached was flown to get a view of the terrain (checking for any unforeseen obstacles) and an idea of the height of the trees. Figure 5.8 shows that most trees are about the same height (approximately $17 \mathrm{~m}$ ) but there are some coniferous trees that are a few meters taller than the surrounding trees. Topography was also a concern for the flight; as shown in Figure 5.5 there can be a change in elevation of $37 \mathrm{~m}$ along a survey line. Since the SkyLance UAS is still in development, a safe flying altitude above the ground level of $80 \mathrm{~m}$ on average (approximately 170m ASL) was chosen. This flight altitude ensured that the aircraft would safely traverse the flight lines without encountering any obstacles. 

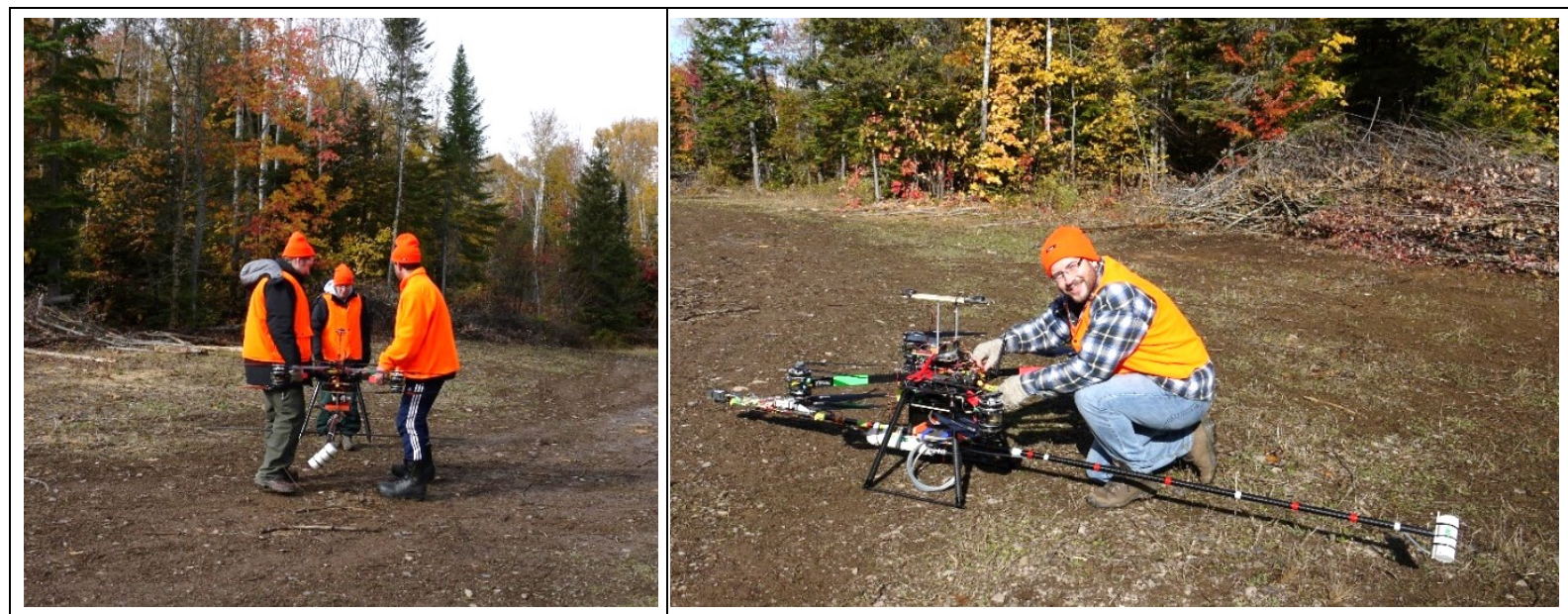

Figure 5.7: The SkyLance UAS survey team. In the left image, from left to right, is Ian Cook, Claire Samson, and Alan Wood. In the right image is Michael Cunningham. 


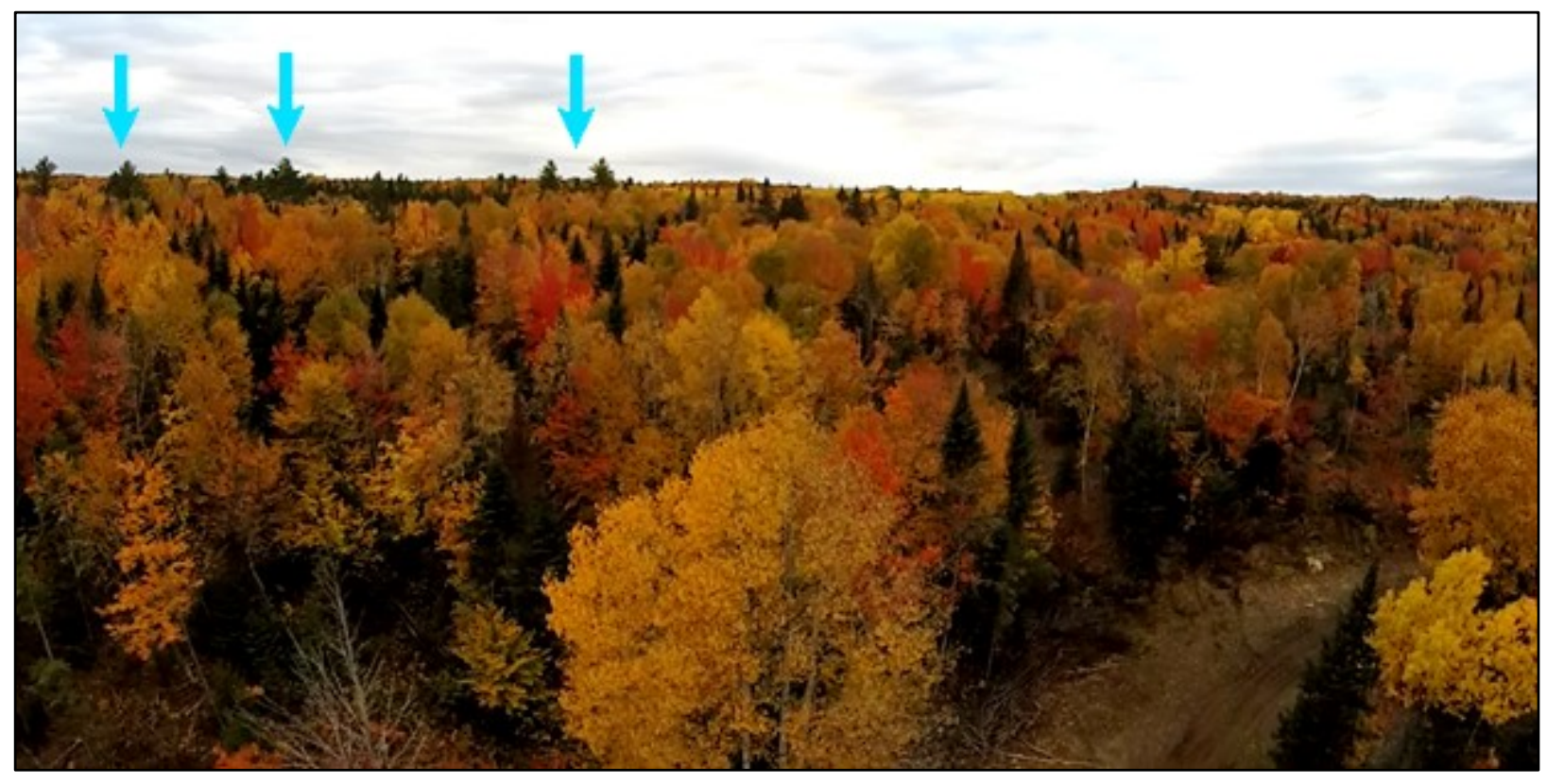

Figure 5.8: Tree-top height variation over the Nash Creek, New Brunswick survey area. There are some trees, pointed out by the blue arrows, which are taller than most of the surrounding trees. Image taken with an IRIS+ UAS using a gimbal stabilized GoPro camera. 
Due to poor weather on October 16, 2015 causing the SkyLance UAS to be unable to fly, time constraints for the survey, and deteriorating weather conditions on October 17, 2015, only three flights were performed with the SkyLance UAS along line 1450 (Figure 5.9) on October 18, 2015. On that day, the weather started out sunny in the morning and as the day progressed the weather became overcast. Wind speeds were low, however, there were the occasional wind gusts. Space Weather Canada reported quiet solar activity for the sub-auroral zone where the survey was located; minimal magnetic noise would be due from solar activity.

Each of the three SkyLance UAS flights covered a further distance than the previous. Prior to the first flight, the system was allowed to idle while recording, initially with the rotors off for approximately 2 minutes and then with the rotors on and the system hovering for another 2 minutes. The mean flight altitude (ASL) and attitudes (pitch, roll, and yaw), with their respective standard deviations are listed in Table 5.2. Flight altitude was stable; only a $\pm 1 \mathrm{~m}$ (standard deviation) in altitude along the flight lines. The flight attitudes were also consistent, with approximately $\pm 5^{\circ}$ variation in pitch, and $\pm 2^{\circ}$ in roll which is primarily associated with wind gusts. The average pitch is negative due to the flight mechanics of a helicopter; pitch needs to point its nose down for the helicopter to fly forwards. The altitude (ASL) is shown in map view along the survey line for each flight in Figure 5.10; the flight altitudes range between $163 \mathrm{~m}$ and $170 \mathrm{~m}$.

The raw magnetic data for these three flights are presented in map view (Figure 5.11) and profile view (Figure 5.12). The first flight (blue) only covered about 
$1 / 3$ of the flight line, starting from the east end of the line heading west. This flight was primarily to test system functionality. The second flight (red) covered approximately $1 / 2$ the flight line. At the end of flight 2 the system lost contact with the home base, causing it to return. By adjusting the home base hardware conditions, the radio strength was improved. Flight 3 (black) was able to cover the full survey line before it lost contact with home base. Data processing of these three flights will be presented in Section 5.2.2. 
Table 5.2: Mean flight altitude and attitudes with 1 standard deviation for each of the three SkyLance UAS flights. Considers only flight along actual survey line shown in Figure 5.9.

\begin{tabular}{|c|c|c|c|}
\hline & Flight 1 & Flight 2 & Flight 3 \\
\hline Mean Altitude (ASL) (m) & $165.3 \pm 1.2$ & $166.3 \pm 1.1$ & $167.2 \pm 1.1$ \\
\hline Mean Pitch (o) & $-4 \pm 4$ & $-6 \pm 5$ & $-6 \pm 5$ \\
\hline Mean Roll (o) & $1.5 \pm 2.3$ & $1.3 \pm 2.5$ & $1.8 \pm 1.8$ \\
\hline Mean Yaw (o) & $278 \pm 9$ & $274 \pm 6$ & $275 \pm 5$ \\
\hline
\end{tabular}




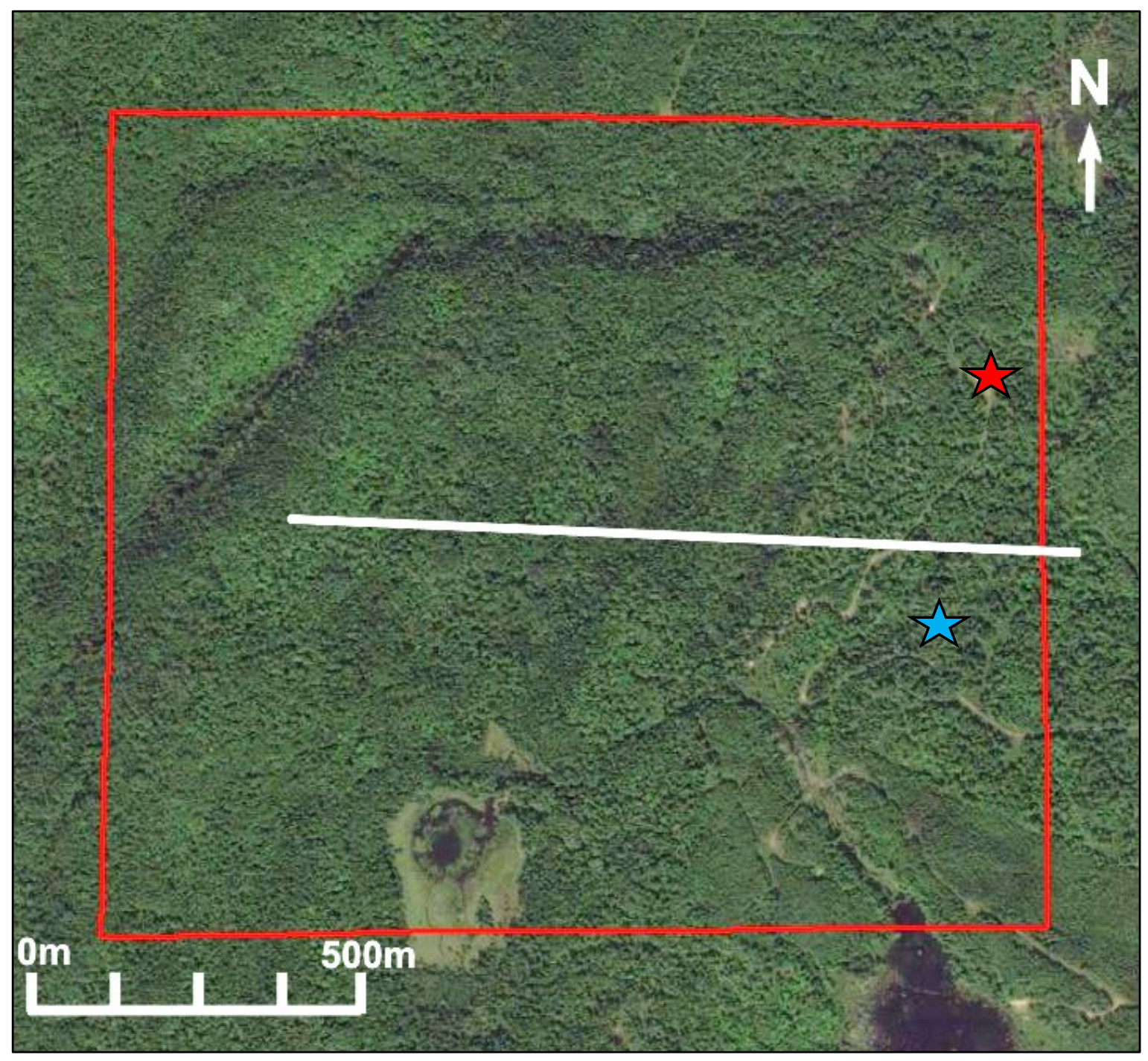

Figure 5.9: Full flight line 1450 flown by the SkyLance UAS on October 17, 2015 (flight 3). The red star is the take-off and landing location. The blue star is the magnetic base station location. Image Source: Google Earth. 


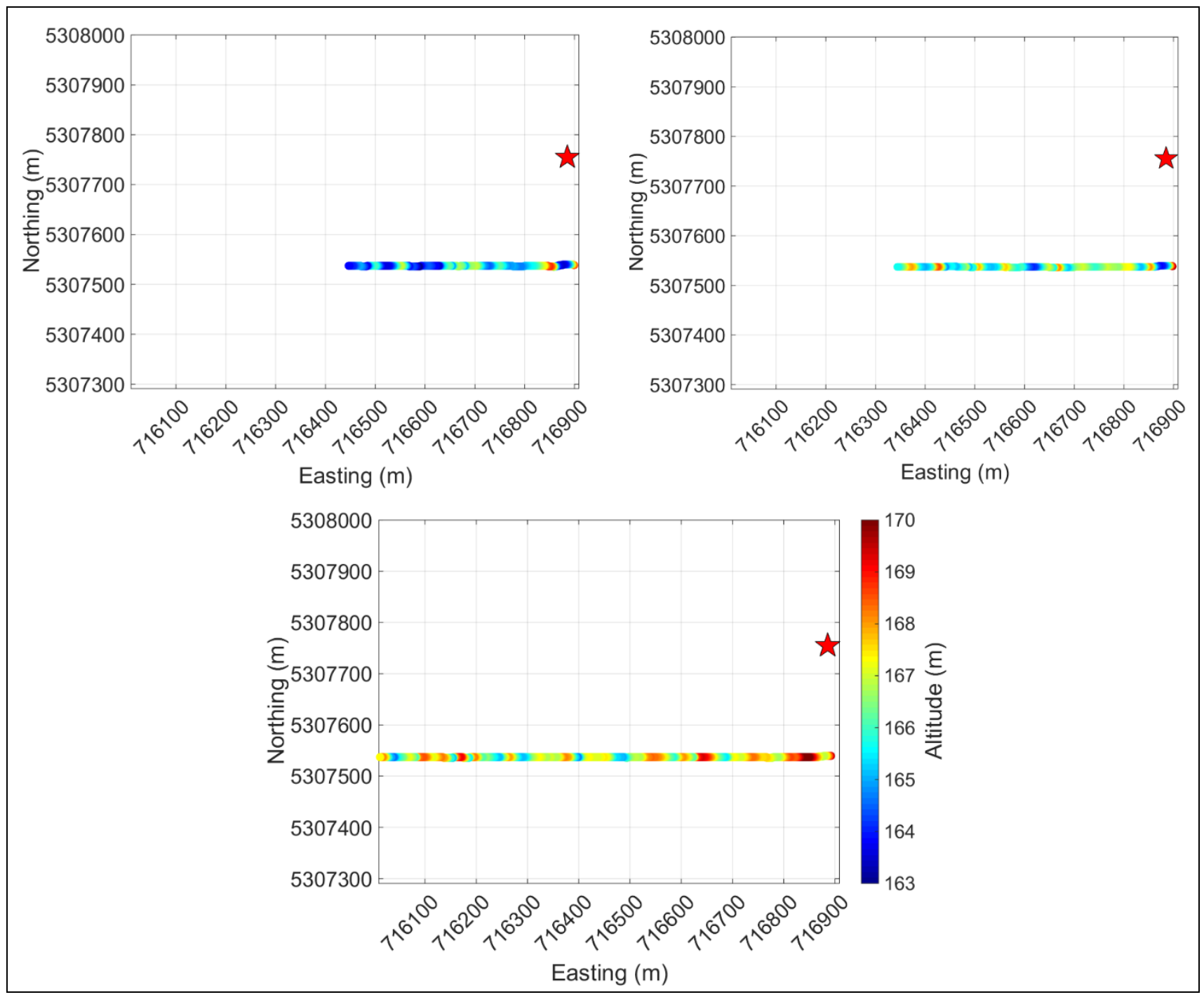

Figure 5.10: SkyLance UAS flights on October 17, 2015 showing altitude variation along the survey line. Flight 1 - top-left plot; Flight 2 - top-right plot; Flight 3 - bottom plot. The red star is the take-off and landing location. 


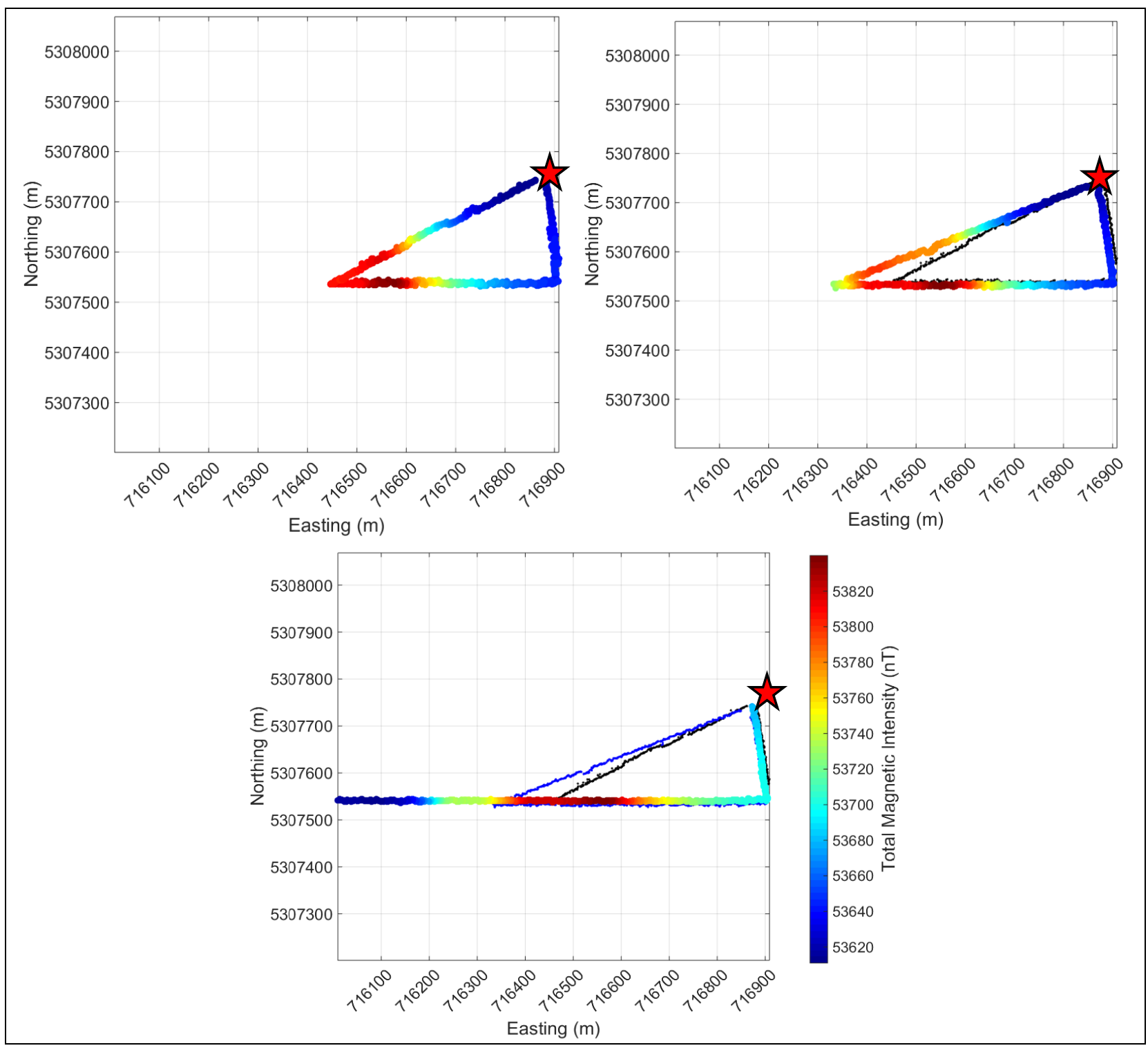

Figure 5.11: Raw TMI data acquired by the SkyLance UAS on October 17, 2015. Flight 1 - top-left plot and also shown as black scatter in the other plots; Flight 2 - top-right pot and also shown as blue scatter in the bottom plot; Flight 3 - bottom figure. The red star is the take-off and landing location. 


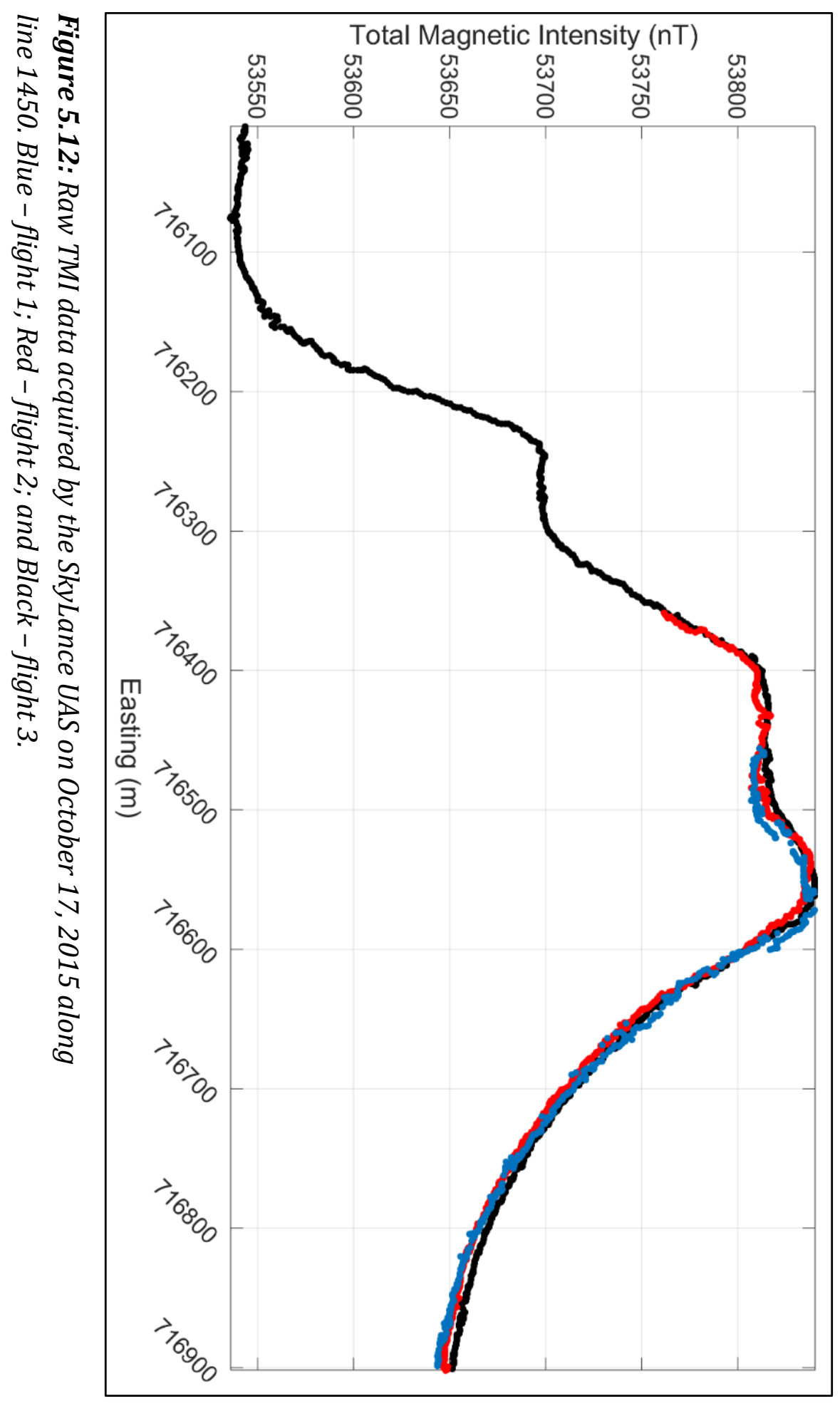




\section{2 - Data Processing}

In this section, both the ground magnetic and aeromagnetic survey data from the Nash Creek, New Brunswick mineral exploration property is processed.

\subsection{1 - Ground Magnetic Survey}

For the three days during which the ground magnetic survey was performed, magnetic base station data was recorded for the application of diurnal corrections. Figure 5.13 presents the base magnetic data where the blue represents day 1 (August 11, 2015), red is day 2 (August 12, 2015), and black is day 3 (August 13, 2015). Over the full set of base magnetic data the average TMI was 53,686nT with a standard deviation of $14 \mathrm{nT}$. There was a minimum and maximum TMI of 53,660nT and $53,735 \mathrm{nT}$, respectively, providing a maximum variation of $75 \mathrm{nT}$ over the full threeday duration. The raw ground magnetic survey data, shown in Figure 5.6, has a maximum variation of 8,188nT (minimum of 50,306nT and maximum of 58,494nT). This causes the adjustments due to the diurnal correction to be fairly negligible in comparison to the detected anomalies; however, diurnal corrections were still applied.

In Figure 5.6 borehole casings from previous exploration drilling are shown as black diamonds. The casings can produce a magnetic signature, so it is necessary to remove the magnetic data around each casing. Any data point that fell within a $15 \mathrm{~m}$ radius of a borehole was removed. As mentioned in Section 5.1.1, GPS signal was lost at various points. In order to remedy this issue, the recorded magnetic data position 
was interpolated along the walking paths when there was no GPS signal. It is also seen in Figure 5.6 that there is large scatter in some of the GPS data; these data were discarded. The data around the location of the parked cars and along the path that extends southward beyond the survey area were also discarded. The GPS position while walking was smoothed to further reduce positional scatter by using an exponential smoothing function. The resultant magnetic data along the walking path is shown in Figure 5.14, where the TMI was limited to between 53,000nT and $55,000 \mathrm{nT}$ to allow for a clearer representation of the magnetic anomalies.

The region highlighted by the red box in Figure 5.14 is part of an access road that was sampled repeatedly on each of the survey days. Figure 5.15 shows the corrected magnetic data along with a linear fit for each of the survey days. This shows that there is approximately a $13 \mathrm{nT}$ shift in the TMI data from day 1 to day 3 , potentially representing instrument drift of the Overhauser magnetometer used.

By performing interpolation of the ground magnetic data shown in Figure 5.14, using the minimum curvature interpolation algorithm developed through the use of MATLAB, a final, fully processed ground magnetic map was produced (Figure 5.16). This map also has the TMI scale limited to between $53,000 \mathrm{nT}$ and $55,000 \mathrm{nT}$ to allow for an easier visual interpretation of where three prominent magnetic anomalies are located. Note there are some discontinuities along the walking paths in this magnetic map. These are possibly related to artefacts produced from the interpolation algorithm used as well as drift effects with the Overhauser 
magnetometer over the three survey days. Figure 5.17 shows a profile extracted from the map shown in Figure 5.16 along line 1450 which intersects the three anomalies. 


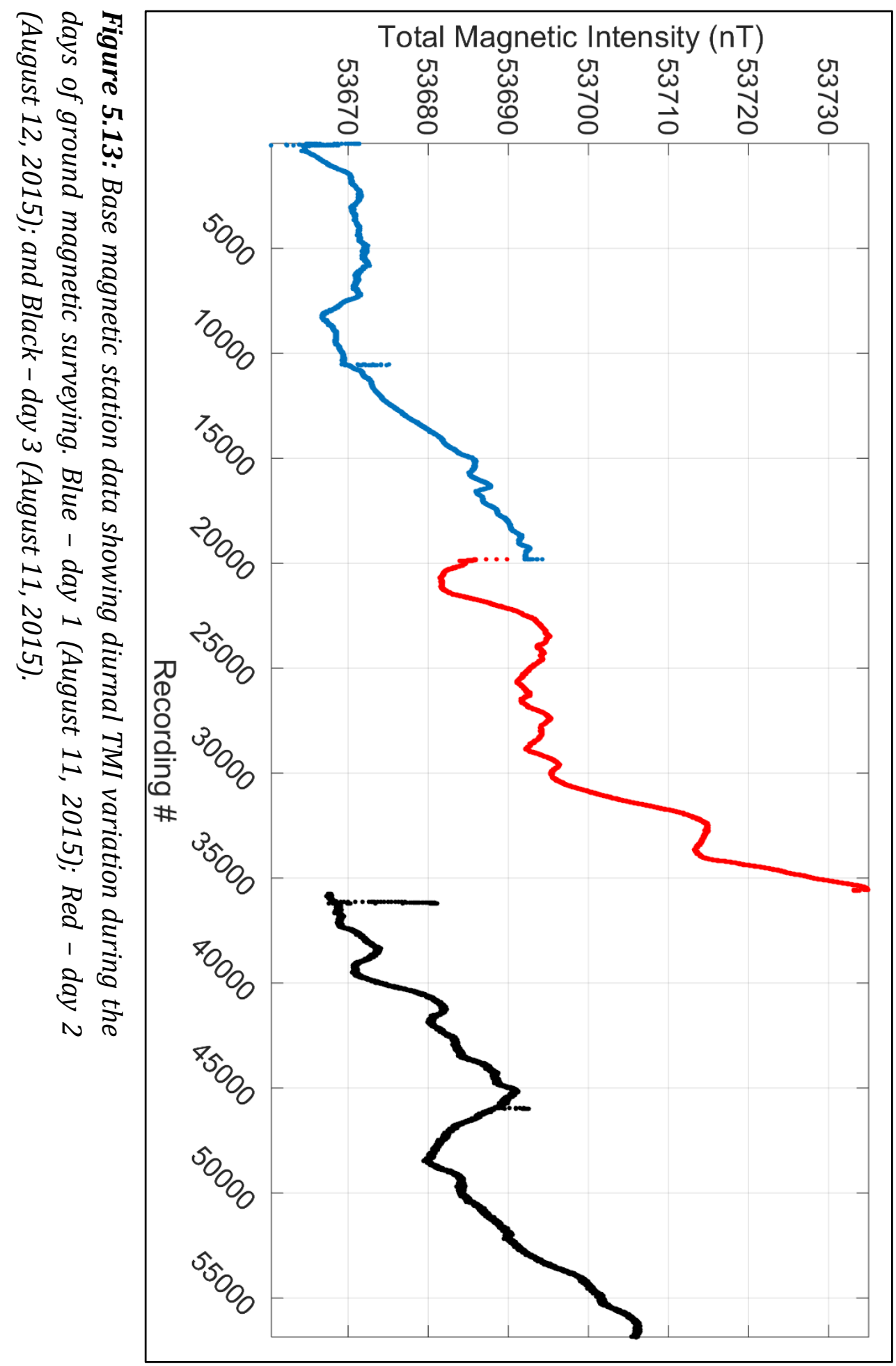




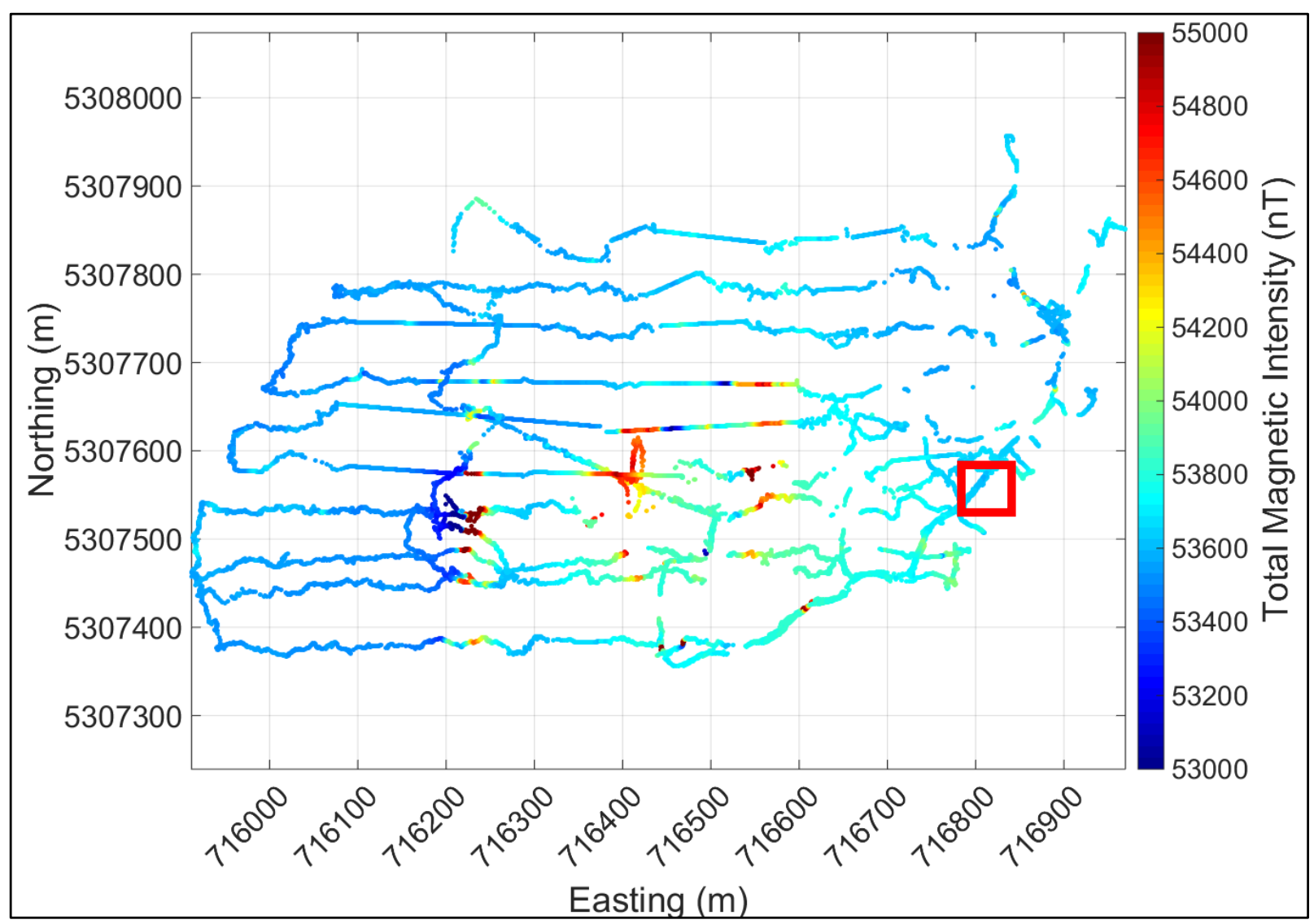

Figure 5.14: Processed ground magnetic data along the walking path. TMI is limited to between 53,000nT and 55,000nT instead of the maximum range of 50,306nT to 58,494nT. 


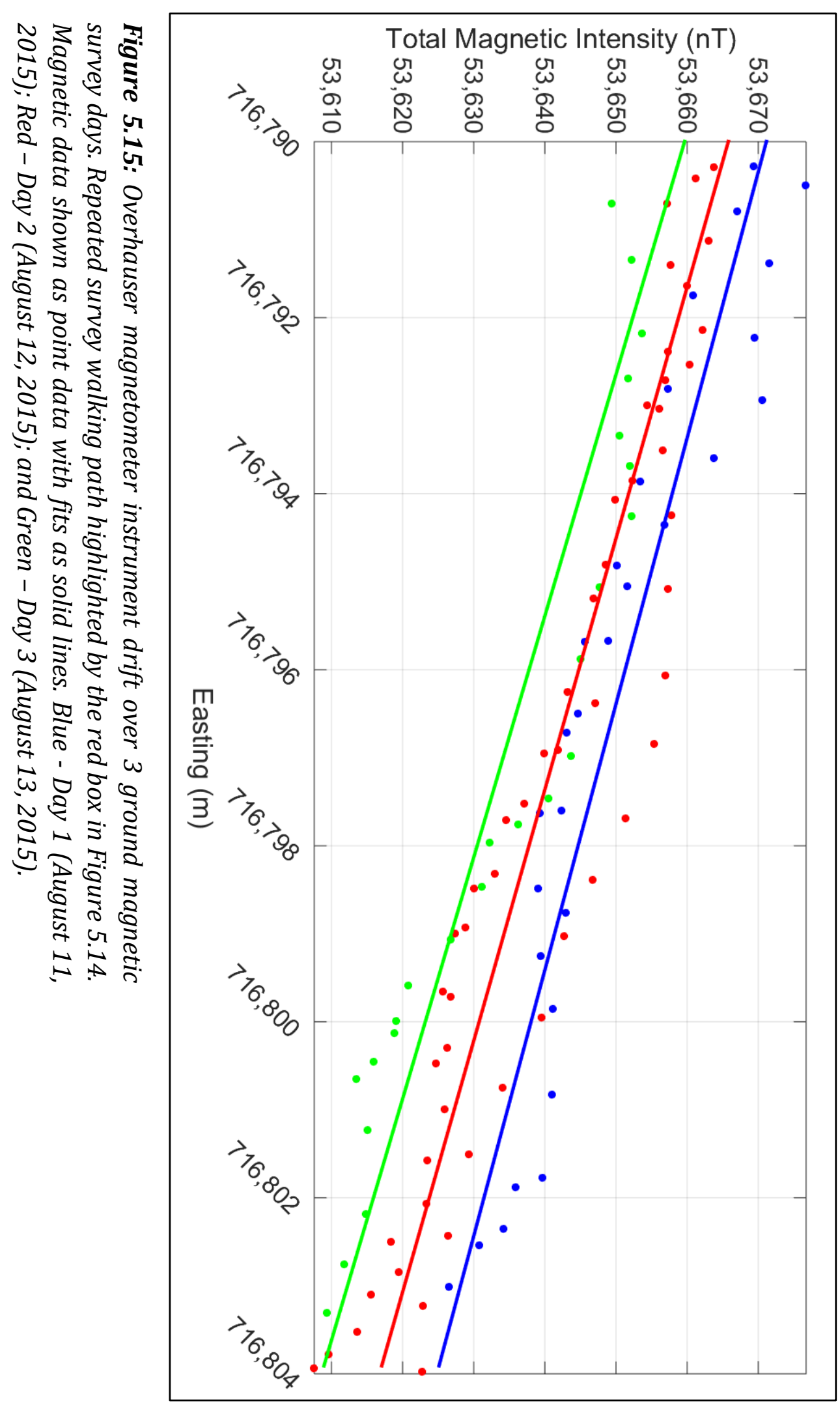




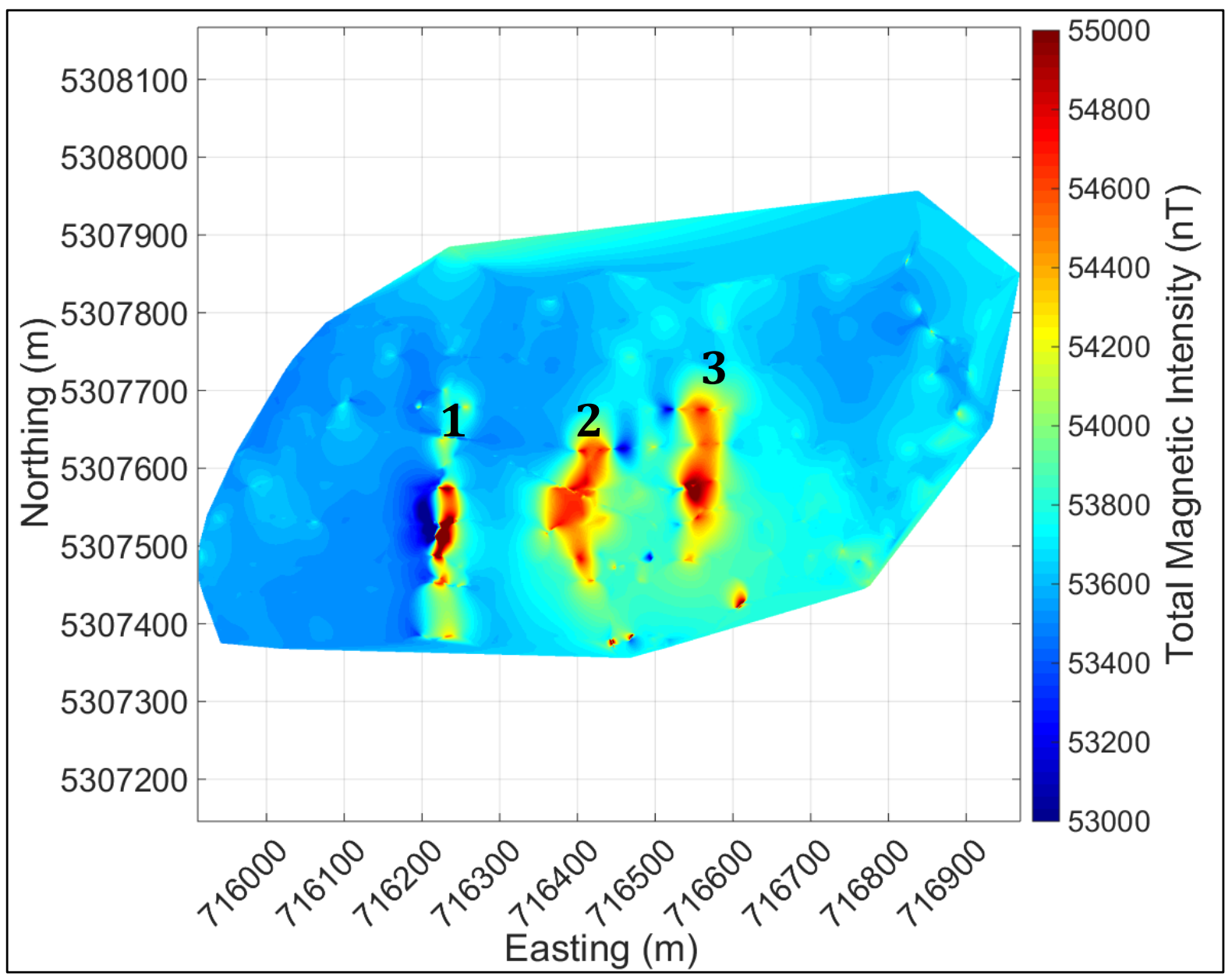

Figure 5.16: Final ground magnetic map for Nash Creek, NB, exhibiting three prominent anomalies labelled 1, 2, and 3. TMI is limited to be between 53,000nT and $55,000 n T$ instead of the maximum range of 50,306nT to 58,494nT. 


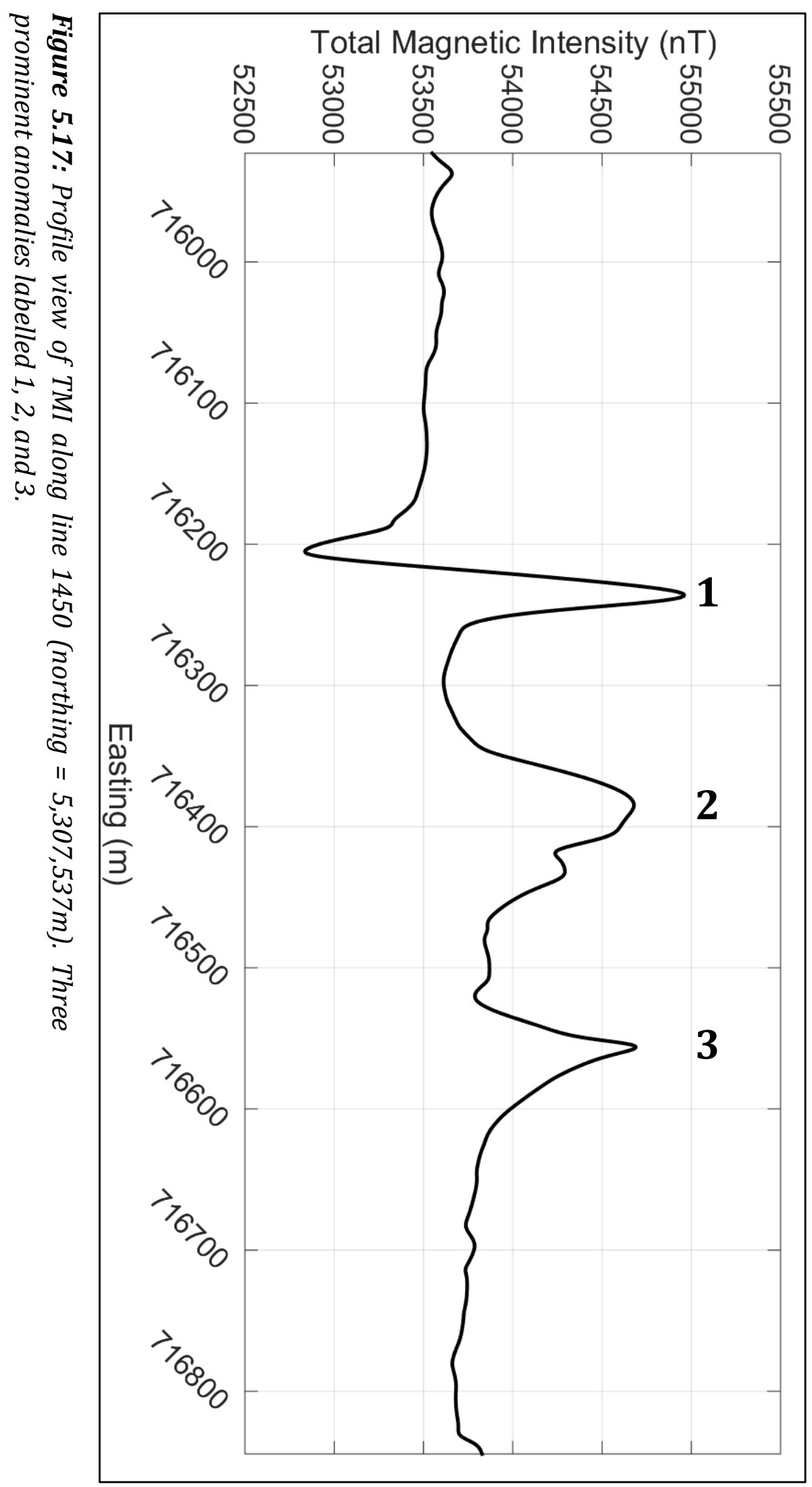




\subsection{2 - Aeromagnetic Survey}

The SkyLance UAS aeromagnetic survey was performed on October 17, 2015. Prior to the flights, the system was allowed to idle without and with the rotors on. Figure 5.18 shows the effects of, first having the rotors off (red) and then turning them on (green) and letting the SkyLance UAS hover. The time at which the power to the rotors is initiated is shown in blue. It is seen that there is a drop in TMI of approximately $0.5 \mathrm{nT}$ when the rotors are turned on. This drop is believed to be related to the orientation of rotors and to the direction of magnetic field they produce, which results in a slight reduction of the Earth's magnetic field. Other than this small drop in TMI there is not a significant in the TMI due to the status of the rotors. After the rotors are initially turned on, shown as green in Figure 5.18, there is over a 150s period a $0.3 \mathrm{nT}$ increase in TMI. This may be caused by a combination of effects such as: increased rotor rotation speeds; increased power output to the rotors; static magnetization of on-board components of the SkyLance; the motions of the SkyLance UAS (i.e. ascending or vibrations); and/or proximity to equipment or vehicles on the ground.

The fourth difference was first calculated for each of the flights (Figure 5.19). The maximum variation is approximately $\pm 0.4 \mathrm{nT}$ with one standard deviation of $\pm 0.04 \mathrm{nT}$. The noise envelope is one order of magnitude higher than the industry standard of $\pm 0.05 \mathrm{nT}$ as laid out by the Geological Survey of Canada (Coyle, M., et al., 2014). As the development of the SkyLance UAS continues, this issue will be mitigated. Some possible solutions to reduce noise include: (1) to optimize the 
magnetometer position; (2) to perform attitude compensation (which is typically done for manned aeromagnetic surveys); and (3) to increase the length of the arms holding the rotors to provide a more stable flight (to reduce oscillations during flight).

The TMI at the magnetic base station over the survey period is shown in Figure 5.20. Because the flights were short in duration there was not a large variation in magnetic field due to diurnal effects; for flight 1 , there were maximum variations of $0.82 \mathrm{nT}, 0.35 \mathrm{nT}$, and $1.04 \mathrm{nT}$ for flights 1,2 , and 3 , respectively. The diurnal corrections applied to the aeromagnetic data had a negligible effect on the overall data.

Due to wind gusts, the attitude (pitch, roll, and yaw) of the SkyLance UAS would occasionally oscillate causing a transient high frequency noise in the aeromagnetic data. This effect is shown in Figure 5.21 and Figure 5.22. This high frequency noise was removed for each flight via filtering, as shown in Figure 5.22.

After the diurnal correction and low-pass filtering were performed the flight line data was smoothed to remove some scatter in the GPS data. Figure 5.23 shows the processed data for each of the flights. The TMI data shows that very high repeatability was achieved along the sections of the line that were covered by each flight. 


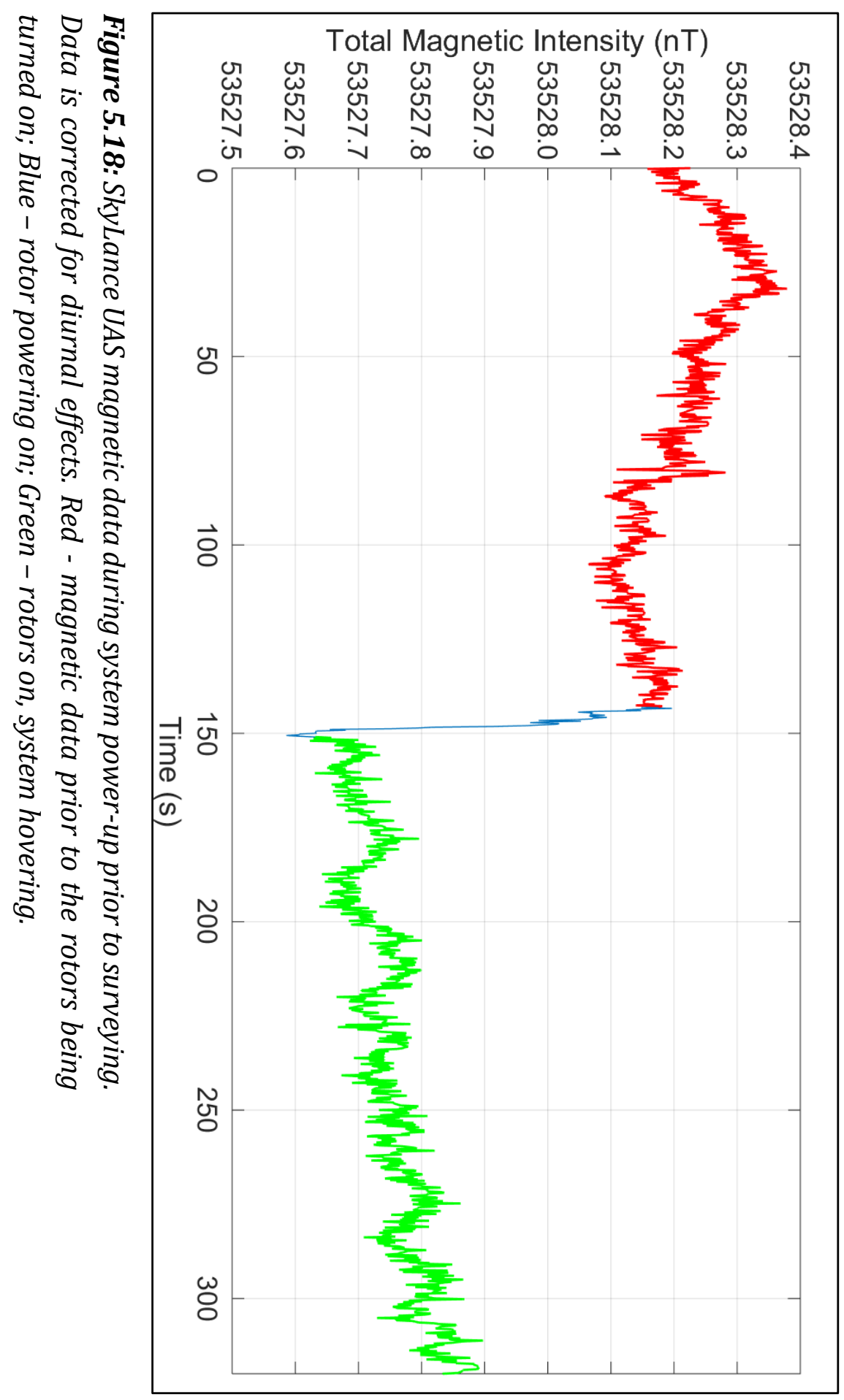




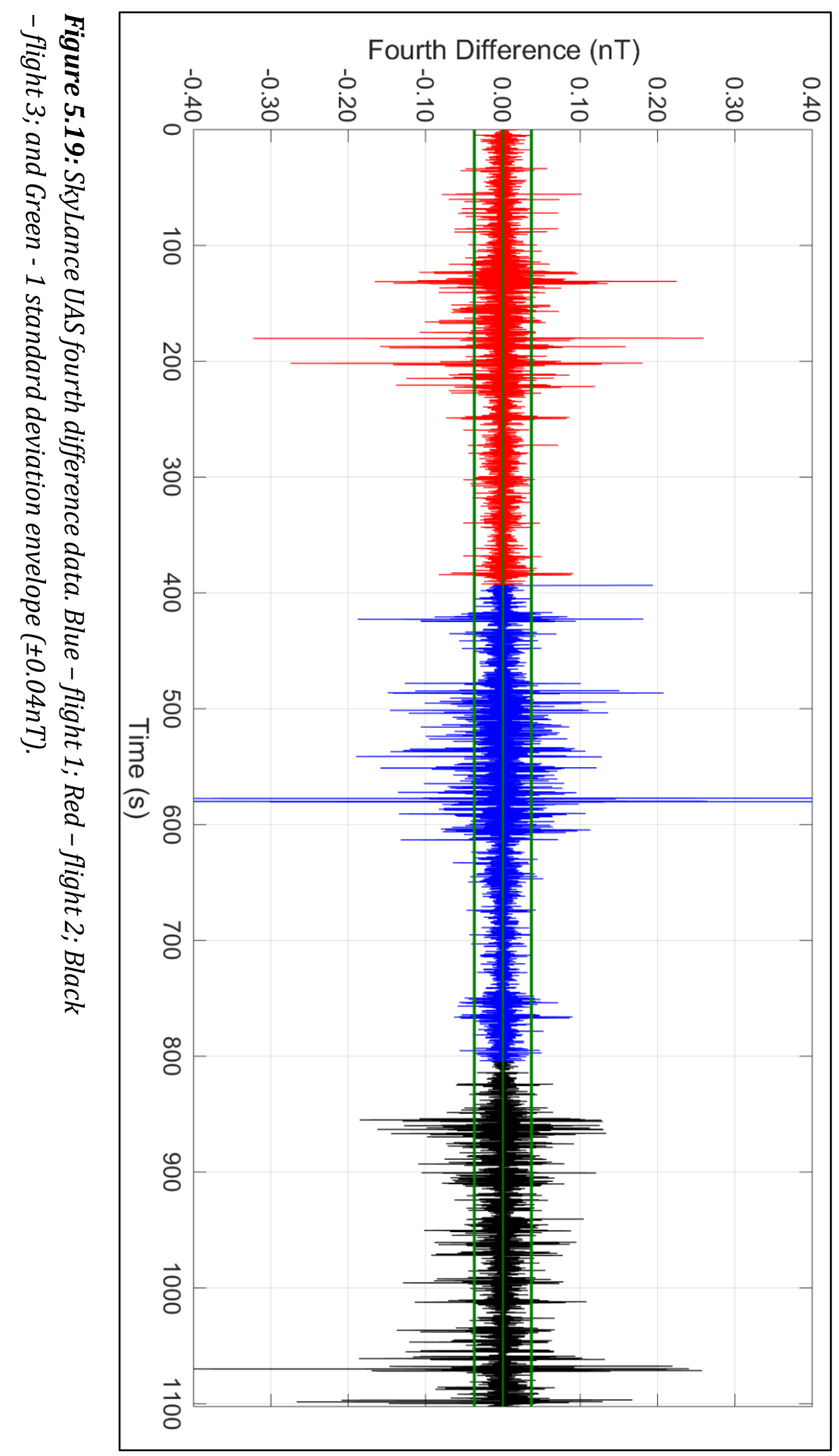




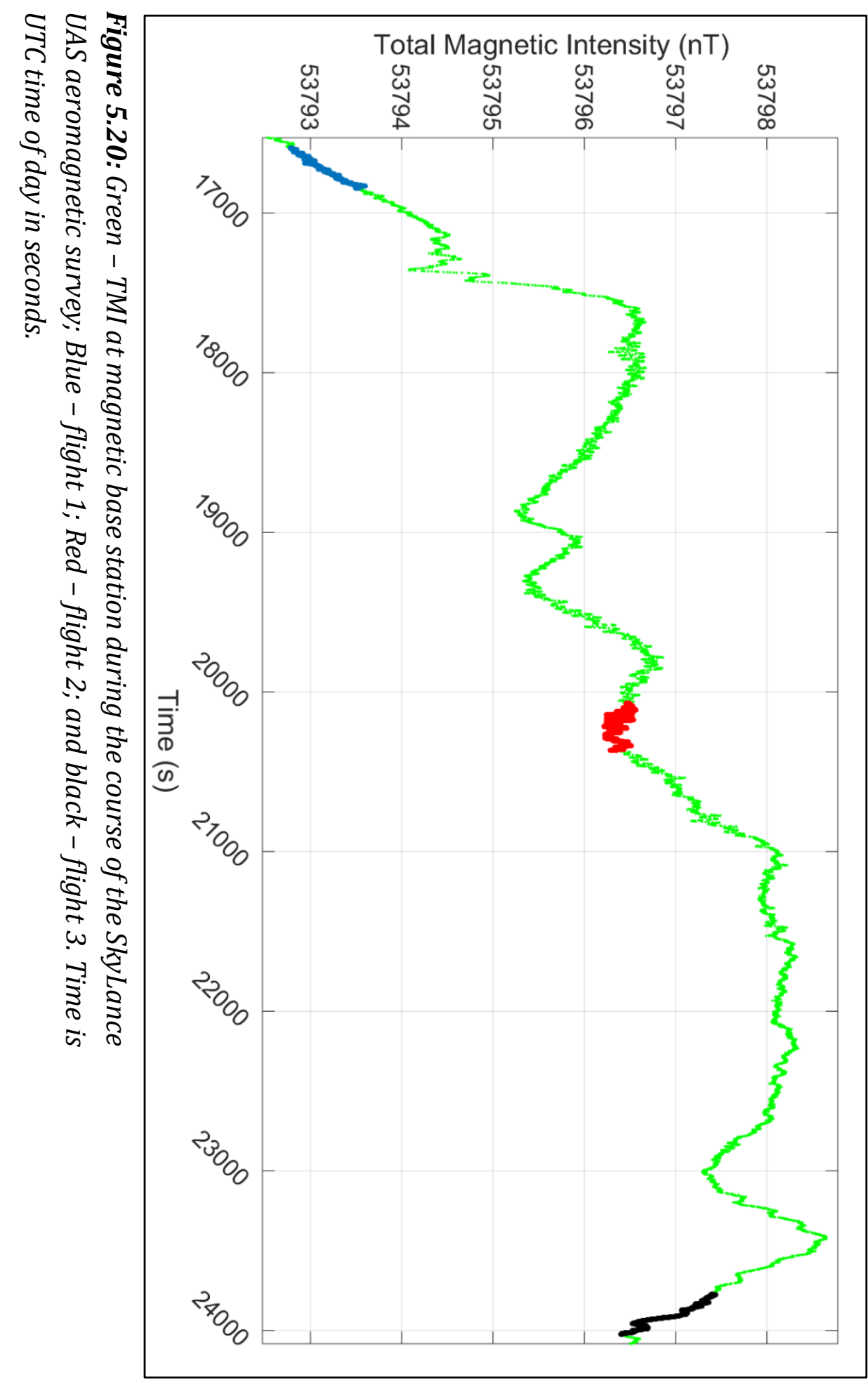




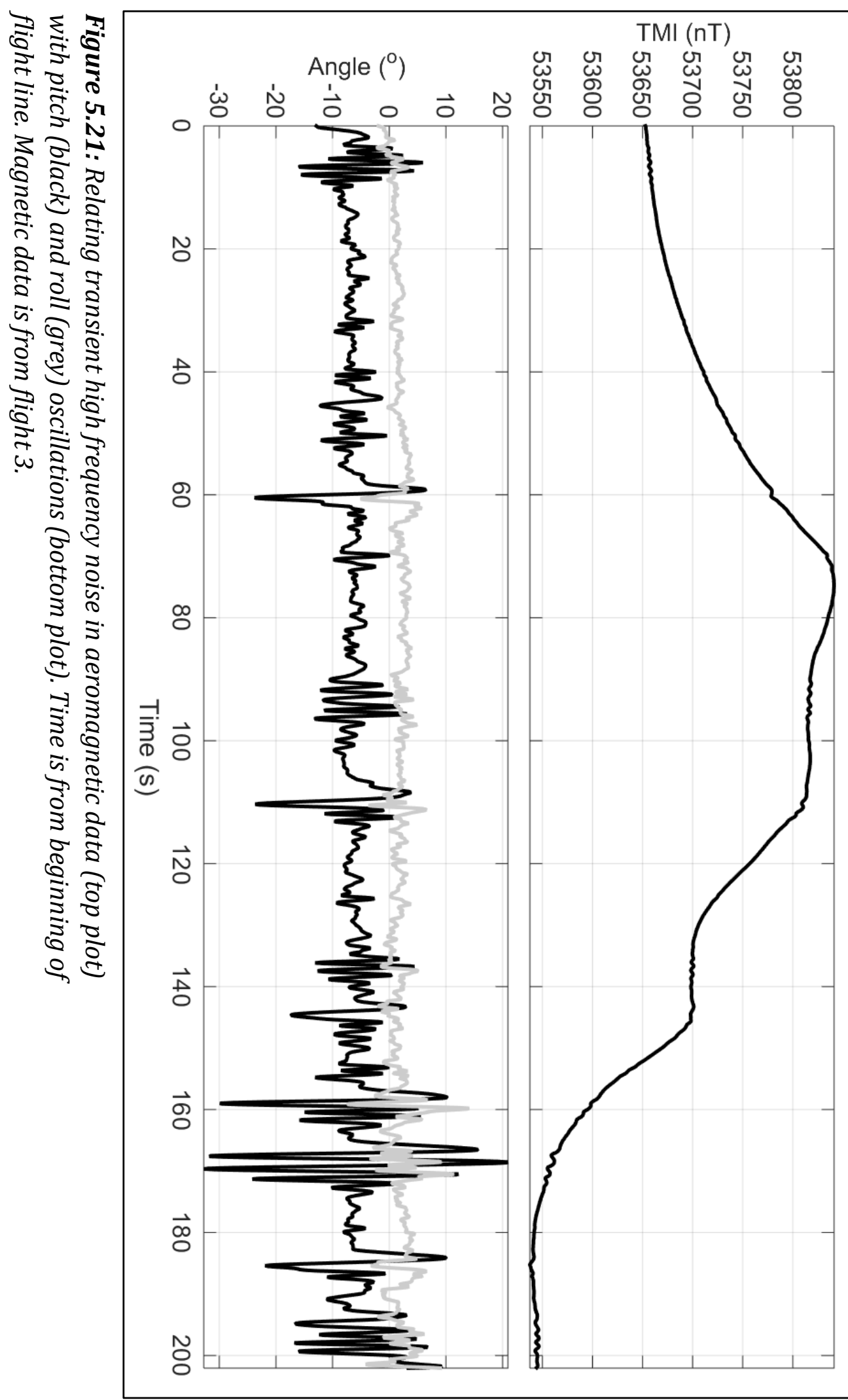




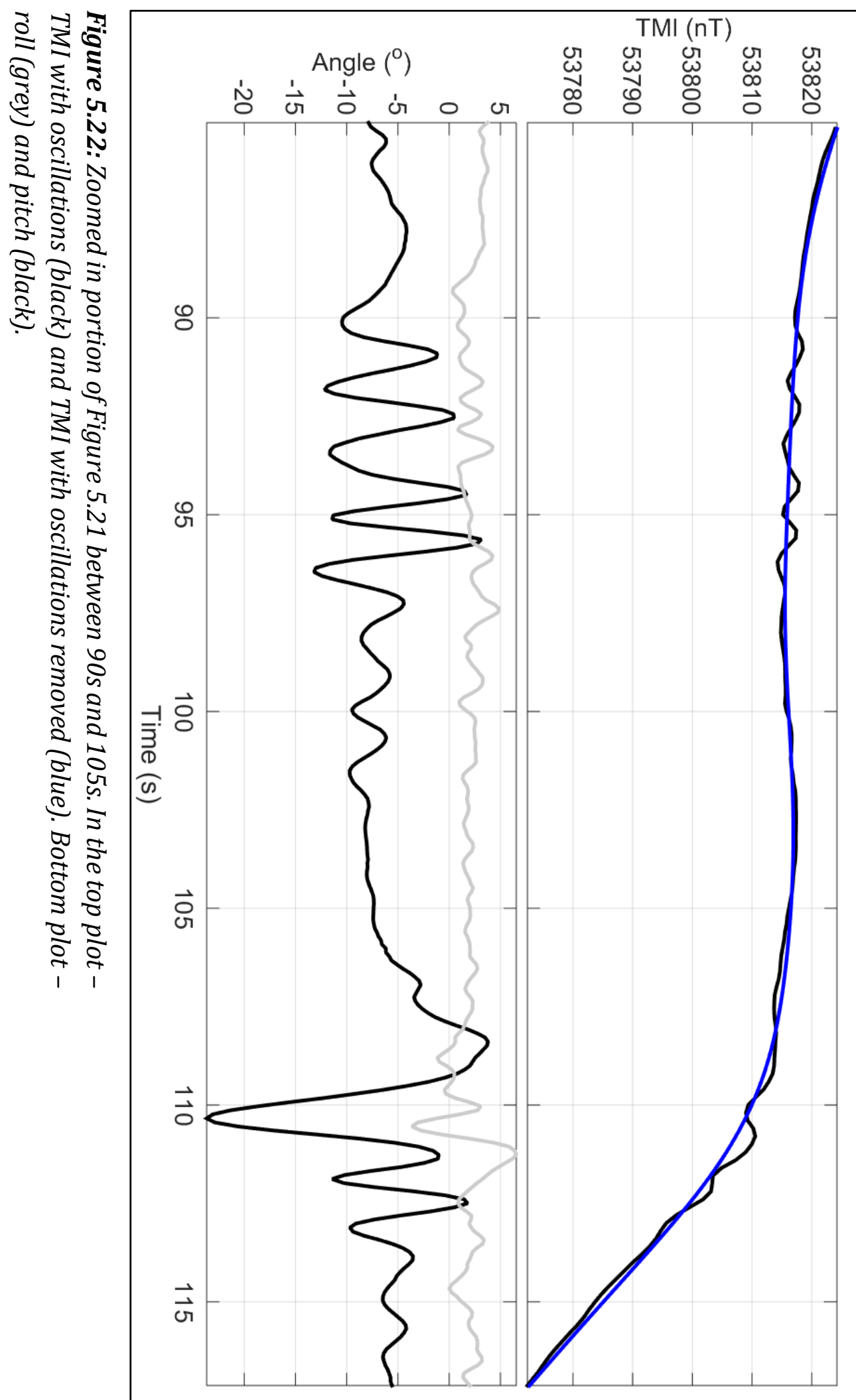




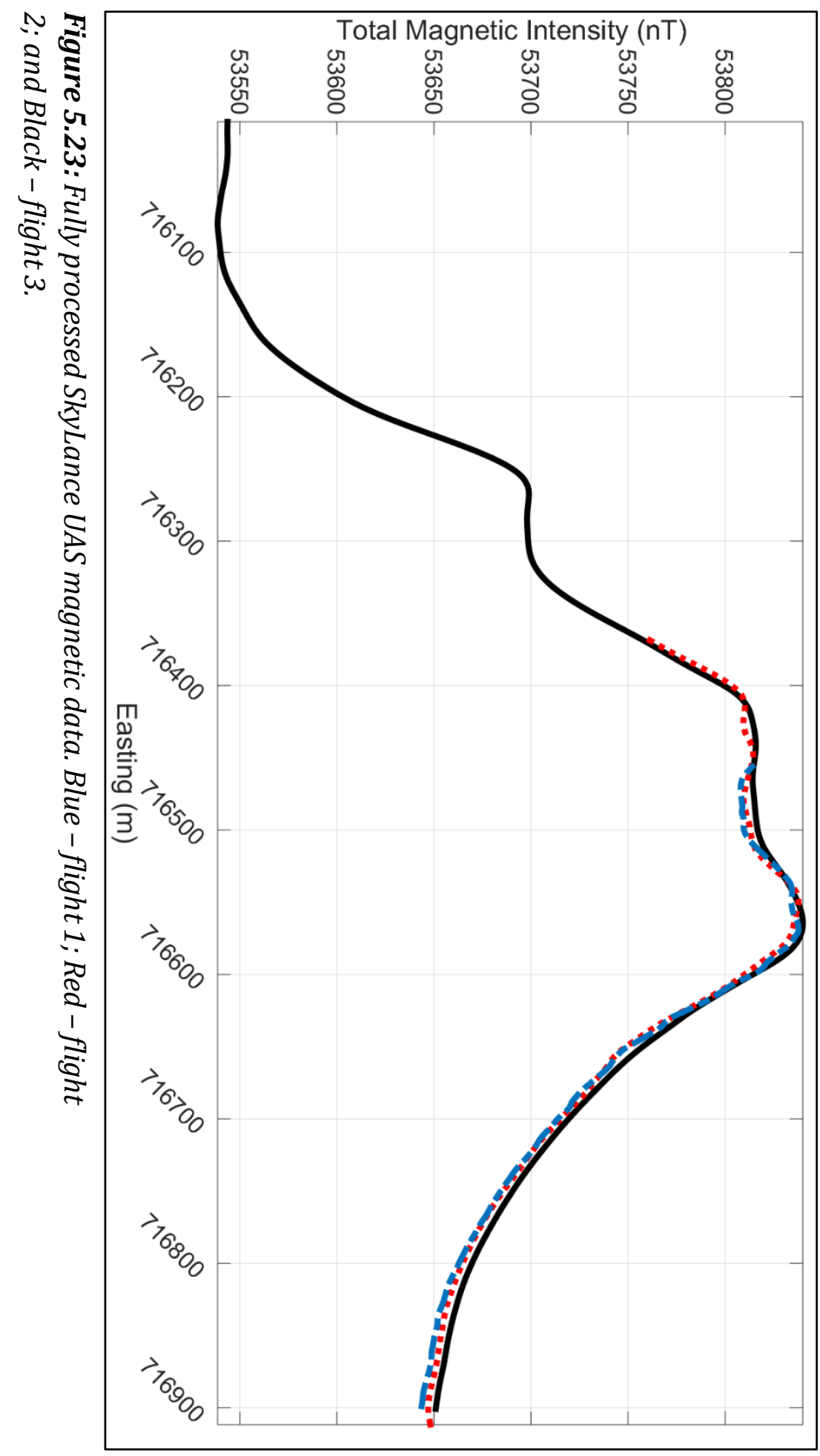




\subsection{3 - Ground Magnetic and Aeromagnetic Survey Comparison}

Upward continuation of the ground magnetic data was performed to allow comparison between ground magnetic and aeromagnetic data recorded by the SkyLance UAS. The ground magnetic data was upward continued (using Geosoft's Oasis Montaj) in $20 \mathrm{~m}$ increments from $0 \mathrm{~m}$ to $100 \mathrm{~m}$ to show continuation trends (Figure 5.24). On the set of upward continuation it can be seen that anomaly 1 is nearly lost at a flight altitude of $60 \mathrm{~m}$ above ground. The anomaly might be shallow in nature and its associated signal is lost with the use of upward continuation. It could also be blending structurally at depth with the other two anomalies. Both anomalies 2 and 3 blend together as the upward continuation height increases. At an average altitude of $80 \mathrm{~m}$ AGL, all three anomalies become too closely spaced for them to be individually detectable.

The SkyLance UAS flew at an altitude of approximately $170 \mathrm{~m}$ ASL, which is on average $80 \mathrm{~m}$ AGL. To match this average flight altitude of the SkyLance UAS an upward continuation of $80 \mathrm{~m}$. Using the $80 \mathrm{~m}$ upward continuation magnetic map (Figure 5.24E) a comparison between the processed ground and flight 3 data is presented in Figure 5.25. The data from line 1450 for the three flights is compared with the same line from the ground magnetic map in Figure 5.26, where the magnetic anomalies previously identified in the ground magnetic data match up well with the SkyLance UAS magnetic data. 


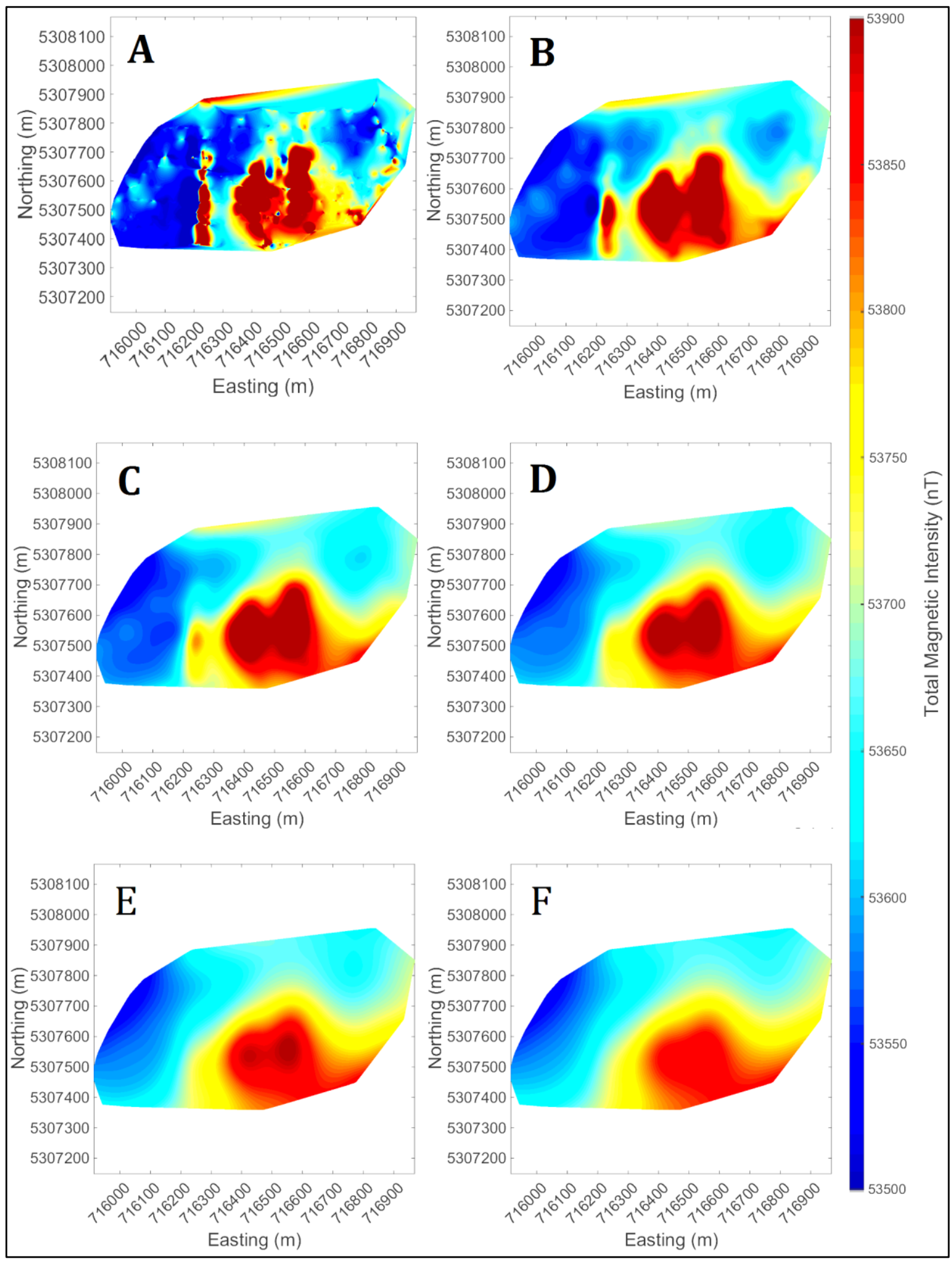

Figure 5.24: Upward continuations of ground magnetic map for Nash Creek, NB. Each TMI plot uses the same magnetic intensity limits for consistency; however, some may go below or above the 53,500nT and 53,900nT range. $A-$ no continuation; $B-20 \mathrm{~m}$; $C$ 40m; D-60m; E-80m; and F-100m. 


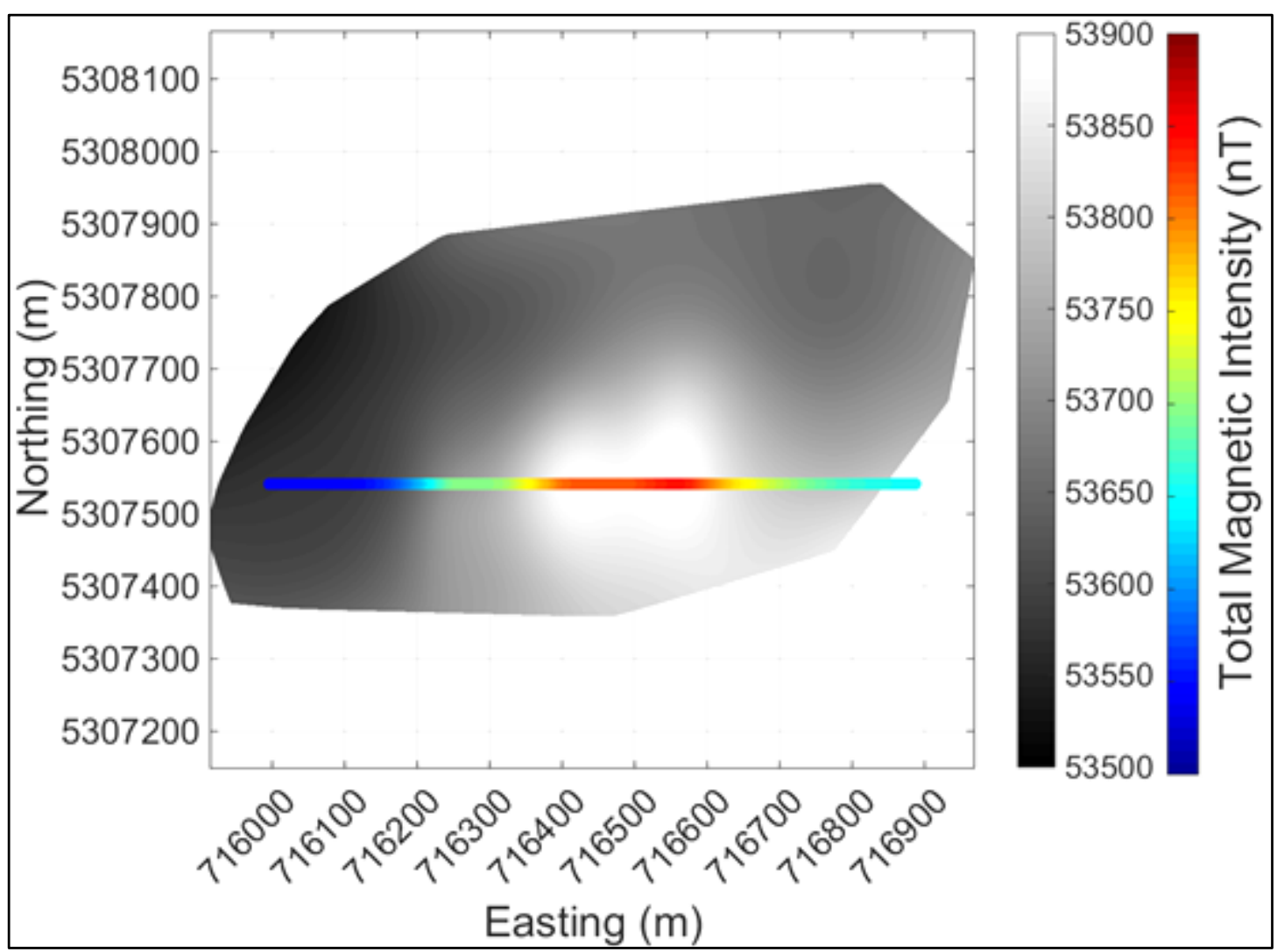

Figure 5.25: Fully processed SkyLance UAS flight 3 aeromagnetic data (coloured) overlaying the ground magnetic data upward continued to 80m AGL (black and white). 


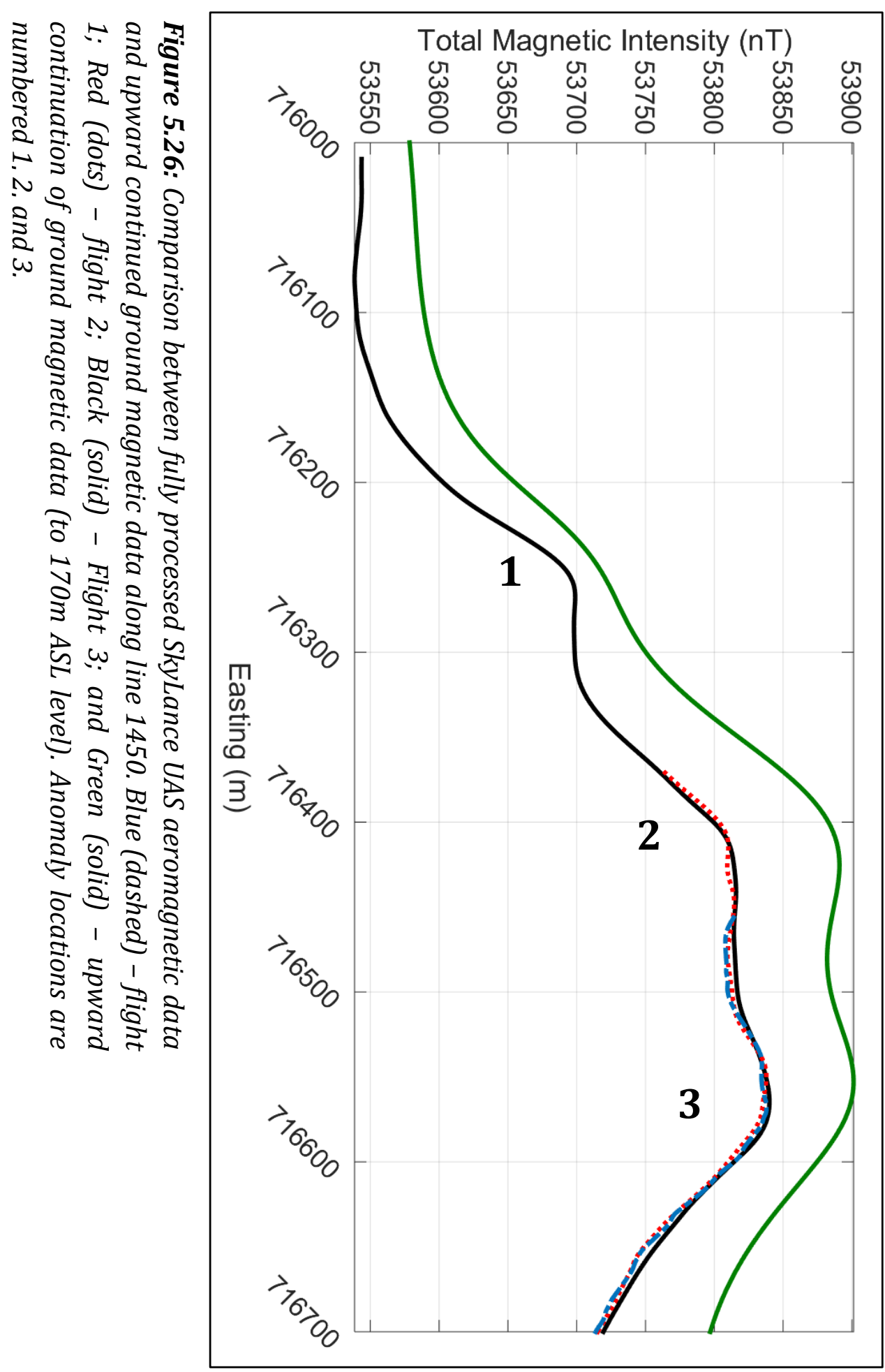




\section{3 -Discussion}

The ground magnetic survey performed in August, 2015, successfully captured and identified the magnetic anomalies which were previously identified, and are labeled in Figure 5.2. Since the ground magnetic data has a higher spatial resolution than the aeromagnetic data in Figure 5.2, it better delineates three distinct magnetic anomalies. These three large magnetic anomalies can be seen prominently in Figure 5.16 where they are very linear and trending north-south. The anomalies are located at an easting of approximately: (anomaly 1) 716,225m; (anomaly 2) 716,400m; and (anomaly 3) 716,550m (Map view: Figure 5.16 and profile view: Figure 5.17). Anomaly 1 could be due to a north-south striking fault plane (Reynolds, 2011): from west to east, there is a magnetic low and then a steep increase in TMI towards a maximum. For anomalies 2 and 3, the magnetic signature could correspond to a dyke-like structure. It is also possible that anomaly 2 and 3 could be related to fault planes as well; the magnetic lows that are expected for a fault plane could be masked by the close spacing between the two anomalies. These three anomalies are prime candidates for the comparison between ground and aeromagnetic data. They are closely spaced and exhibit very sharp features, this lets us determine how capable the SkyLance UAS is at identifying and delineating geological magnetic anomalies.

The average above ground flight altitude of the SkyLance UAS is $80 \mathrm{~m}$, so a comparison between the aeromagnetic map and $80 \mathrm{~m}$ upward continuation magnetic map is show in map view for flight 3 in Figure 5.25, and also in profile view for all three flights in Figure 5.26. The trends of the two datasets match very well. There is 
a DC shift of approximately 20-50nT in TMI between the upward continued and SkyLance data. This is possibly due to: (1) an effect from the upward continuation algorithm used; (2) differences in the TMI due to the time of year the surveys were performed (August and October, 2015); (3) static magnetic sources on the SkyLance UAS; and/or (4) instrument drift on the Overhauser magnetometer used to produce the ground magnetic survey data. Anomalies 2 and 3 are very similar in shape; however, anomaly 1 is more pronounced in the aeromagnetic survey data. This is an artifact related to the upward continuation; it was not possible to perform the upward continuation of the ground magnetic data (which varies in elevation across the survey) to a constant altitude ASL to exactly match the aeromagnetic data. In the vicinity of anomaly 1 , the SkyLance UAS is flying closer to the ground than it is over other areas of the survey line.

Overall, both the ground magnetic and aeromagnetic surveys performed at the Nash Creek, NB property were successful. The ground magnetic map provides important insight for further development of the mineral deposit. Ground magnetic data had not been previously acquired on the property. The data outlined three strong magnetic targets, providing results that will help steer future exploration strategies. The SkyLance UAS aeromagnetic survey was successful in delivering high-resolution, repeatable magnetic data. Further development of the SkyLance UAS should focus on improving the data quality so that the fourth difference noise levels fall within the industry standards. Stability during flight is another area for improvement, with the objective of attenuating the high-frequency pitch oscillations occurring during wind gusts. 


\section{6 - Summary and Concluding Remarks}

The research presented in this thesis is focussed on aeromagnetic surveying using UASs, specifically the Venturer fixed-wing UAS, and the SkyLance rotary-wing UAS. These systems have been designed to perform high-resolution aeromagnetic surveys for mineral exploration, to identify metallic ore bodies that have a magnetic signature.

From the theoretical analysis performed in Chapter 3, it was shown that UASs are very capable contenders for aeromagnetic surveying. UASs flying at 50m AGL altitude, such as the Venturer UAS, would detect smaller, less magnetic and deeper ore bodies compared to traditional fixed-wing manned systems typically flying at an altitude of $100 \mathrm{~m}$. Further improvements could be delivered by UASs flying at even lower altitudes, such as the SkyLance UAS. Lowering of flight altitudes allows for increased discrimination of two magnetic bodies closely spaced to one another. If two bodies had a limiting depth of $40 \mathrm{~m}$ and a radius of $10 \mathrm{~m}$, they would need to be spaced at least $67.5 \mathrm{~m}$ apart for a traditional systems to detect them. At a UAS altitude AGL of $50 \mathrm{~m}$, however; the bodies would only need to be separated by $45.0 \mathrm{~m}$ for successful detection while at $2 \mathrm{~m}$ altitude, they would need to be separated by only $22.5 \mathrm{~m}$. Although their wingspan is smaller, theoretical analyses also showed that fixed-wing UASs instrumented with 2 magnetometers on the wingtips have the potential of recording gradiometric data of equivalent quality as fixed-wing manned systems.

Fixed-wing UASs, such as the Venturer, will become accepted by the industry if they can deliver the same data quality as their manned counterparts. The analyses 
presented in this thesis show that the required level of performance is already achieved by the Venturer UAS. This UAS is very stable in flight, keeping the fourth difference levels within industry standards and producing high-quality aeromagnetic surveys. Further improvements will be made when a real-time or post-processing system has been implemented to perform attitude compensation. Currently, one of the main hurdles to market penetration for fixed-wing UAVs with a mass greater than $25 \mathrm{~kg}$ is the complicated application process for the obtention of a Special Flight Operations Certificate from Transport Canada.

The UASs flying at very low altitude, most likely rotary-wing UASs, could eventually replace the ground magnetic survey crew. Since these UASs tend to be limited by battery power, they can only fly shorter distances, however; they are capable of flying much faster than the survey crew can walk. As in the case of ground magnetic surveys, these rotary-wing UASs would also require cut lines prior to surveying unless the survey is performed over barren terrain (i.e. arctic tundra, frozen lakes, and desert) or flown above the vegetation. The SkyLance rotary-wing UAS is an example of a small $(<25 \mathrm{~kg})$ rotary-wing UAS. This research project demonstrated that the SkyLance is capable of acquiring very repeatable data. It was further shown that the survey results compare very well with the upwardly continued ground magnetic data except for a 20-50nT shift in TMI. The SkyLance UAS is still in its developmental stages, so further improvements will be forthcoming. The fourth difference noise levels for the SkyLance UAS ranged between $\pm 0.4 \mathrm{nT}(0.8 \mathrm{nT}$ peak to peak), which is higher than the industry's acceptable level of $\pm 0.05 \mathrm{nT}(0.1 \mathrm{nT}$ peak to peak). There are various options to remedy this issue, and some are already in the 
works. Due to the length of the magnetometer pole, it is challenging to keep the system stable in flight. An increase in the aircraft footprint, by increasing the arms that hold the rotors would provide a more stable flight. As for the Venturer UAS, the implementation of an attitude compensation system, would reduce noise. A major advantage of UASs with a mass smaller $25 \mathrm{~kg}$, such as the SkyLance, is that the regulatory framework is much simplified.

Overall, this research project has shown that the use of UASs for aeromagnetic surveying might fill a gap between high altitude, traditional aeromagnetic surveys, and ground magnetic surveys. With further development, UASs might eventually replace these two survey methods altogether. UASs are highly versatile systems capable of providing high-quality survey results with details that traditional aeromagnetic systems cannot provide while flying at a fraction of the cost. It is critical, however, that the UASs acquire magnetic data of the same or better quality if they are to become an alternative to traditional surveys. UASs are also appealing systems for future use since they require less fuel than their traditional airborne counterparts and have a smaller environmental footprint. The two UASs featured in this thesis, as well as many others, will continue to advance at a fast pace. It will be exciting to watch how the mineral exploration industry will evolve with the further development of UASs for aeromagnetic surveying. 


\section{7 - References}

Anderson, D., \& Pita, A. (2005). Geophysical surveying with GeoRangerTM UAV. American Institute of Aeronautics and Astronautics Inc., 67-78.

Anderson, J. (2011). Fundamentals of Aerodynamics, 5th Edition. New York: McGrawHill.

Anderson, K \& Gaston K. (2013). Lightweight unmanned aerial vehicles will revolutionize spatial ecology. Frontiers in Ecology and the Environment. $11(2), 138-146$

Barnard, J. (2008). The use of unmanned air vehicles in exploration and production activities. 78th Annual Meeting of the Society of Exploration Geophysicists. 27, pp. 1132-1136. Las Vegas, NV: SEG Expanded Abstracts.

Blakely, R. (1996). Potential theory in gravity and magnetic applications. Cambridge, UK: Cambridge University Press.

Bongajum, E. (2011). Investigating seismic wave scattering in heterogeneous environments and implications for seismic imaging. Toronto: University of Toronto.

Brown, D. (2007). Technical report on mineral resource estimate, Nash Creek project, Restigouche County, New Brunswick Canada.

Burns, M. (2015). UAV Remote Sensing \& Survey Solutions. Retrieved 11 09, 2015, from Pioneer Exploration Consultants Ltd.: http://www.pioneerexploration.ca/uav-airborne-surveys.html

Caron, R., Samson, C., Straznicky, P., Ferguson, S., \& Sander, L. (2014). Aeromagnetic surveying using a simulated unmanned aircraft system. Geophysical Prospecting, 62, 352-363. 
Caron, R., Samson, C., Straznicky, P., Ferguson, S., Archer, R., \& Sander, L. (2011). Magnetic and magneto-gradiometric surveying using a simulated unmanned aircraft system. 81st Annual meeting of the Society of Exploration Geophysists. 30, pp. 861-865. San Antonio, TX: SEG Expanded Abstracts.

Coyle, M., Dumont, R., Kiss, F., \& Miles, W. (2014). Geological Survey of Canada aeromagnetic surveys: design, quality assurance, and data dissemination. Ottawa: Geological Survey of Canada. doi:doi:10.4095/295088

Cunningham, M., \& Samson, C. (2015, November 3-5). Detecting mineral ore bodies with UASs instrumented with magnetometers. Unmanned Systems Canada Conference. Halifax, NS: 3-5 November.

Cunningham, M., Samson, C., Wood, A., Cook, I., \& Doyle, B. (2015). Assessing the capabilities of unmanned fixed and rotary wing systems for geomagnetic surveying. AGU-CGU-GAC-MAC Joint Assembly. Montreal, QC: 3-7 May.

Dion-Ortega, A. (2015, 06 01). Abitibi Géophysique lance le tout premier drone magnétométrique. Montreal, QC, Canada. Retrieved 11 09, 2015, from https://www.lesaffaires.com/dossier/exploration-miniere/abitibigeophysique-lance-le-tout-premier-drone-magnetometrique/579253

Drenth, B. (2014). Geophysical expression of a buried niobium and rare earth element deposit: The Elk Creek carbonatite, Nebraska, USA. Society of Exploration Geophysics, SJ23-SJ33.

Elkins, T., \& Hammer, S. (1938). The resolution of combined effects, with applications to gravitational and magnetic data. Geophysics, 315-331. doi:10.1190/1.1439512

Ferguson, I., Epp, D., Saturnino, T., Orellana, M., Cravn, J., \& Jones, A. (2015). Interpretation of resisitivity and magnetic anomalise from the Fox River Sill, Trans Hudson Orogen, Canada. 24th International Conference and Exhibition (pp. 1-5). ASEG Extended Abstracts. 
Geiger, K.W. (1968). Bedrock topography of the Gleichen Map-Area, Research Council of Alberta, Report 67-2.

Hood, P., \& Teskey, D. (1989). Aeromagnetic gradiometer program of the Geological Survey of Canada. Geophysics, 54(8), 1012-1022.

Johnson, R.A. \& Bhattacharyya, G.K. (2014). Statistics: Principles and Methods, $7^{\text {th }}$ Edition. Wiley.

Keary, P., Brooks, M., \& Hill, I. (2002). An Introduction to Geophysical Exploration. Oxford: Blackwell Publishing.

Kroll, A. (2013). Evaluation of an unmanned aircraft for geophysical survey. 23rd International Geophysical Conference and Expedition (pp. 1-4). Melbourne, Australia: ASEG Extended Abstracts.

Leach, B. (1979). Aeromagnetic compensation as a linear regression problem. National Aeronautical Establishment.

Lee, M. \& Morris, W. (2013). Quality assurance of aeromagnetic data using lineament analysis. Exploration Geophysics. 44, 104-113

Li, Y. \& Oldenburg, D.W., 1998, Separation of regional and residual magnetic field data. Geophysics. 63, 431-439

Luyendyk, A. (1997). Processing of airborne magnetic data. Journal of Australian Geology \& Geophysics, 17(2), 31-38.

Mandea, M., \& Korte, M. (2011). Geomagnetic observations and models. IAGA Special Sopron Book Series 5. Springer Science and Business Media.

Milkereit, B. (2015, August). Personal Communication.

Minty, B. (1991). Simple micro-levelling for aeromagnetic data. Exploration Geophysics, 22, 591-592. 
Natural Resources Canada. Space Weather Canada. Retrieved from Space Weather: http://www.spaceweather.gc.ca/index-en.php. Accessed on August 11-13, 2015.

Pajares, G. (2015). Overview and current status of remote sensing applications based on unmanned aerial vehicles (UAVs). Photogrammetric Engineering and Remote Sensing, 281-329.

Peck, R. \& Devore, J.L. (2012). Statistics - The exploration \& analysis of data, 7th edition. Cengage Learning.

Ranalli, G. (1995). Rheology of the Earth, Second Edition. London: Chapman \& Hall.

Raymer, D. (2006). Aircraft design: a conceptual approach, 4th Edition. Reston, VA: American Institute of Aeronautics and Astronautics.

Reynolds, J. (2011). An Introduction to Applied and Environmental Geophysics Second Edition. West Sussex, UK: Wiley-Blackwell.

Samson, C., Cunningham, M., Wood, A., Cook, I., \& Dyle, B. (2014). Magnetic surveying using an unmanned system for geological mapping. Unmanned Systems Canada Conference. Montreal: 4-6 November.

Samson, C., Straznicky, P., Laliberte, J., Caron, R., Ferguson, S., \& Archer, R. (2010). Designing and building an unmanned aircraft system for aeromagnetic surveying. 80th Annual meeting of the Society of Exploration Geophysicists. 29, pp. 1167-1171. Denver, CO: SEG Expanded Abstracts.

Saul, S., \& Pearson, M. (1998, December). Levelling of aeromagnetic data. Canadian Journal ofExploration Geophysics, 34, 9-15.

Telford, W., Geldart, L., Sheriff, R., \& Keys, D. (1976). Applied Geophysics. Cambridge University Press. 
Teskey, D., Barlow, R., Hood, P., Lefebvre, D., Paterson, N., Reford, M., \& Watson, D. (1991). A guide to aeromagnetic specifications and contracts. Ottawa:

Geological Survey of Canada. Open File 2349.

Tollo, R., Corriveau, L., McLelland, J., \& Bartholomew, M. (2004). Proterozoic tectonic evolution of the Grenville orogen in North America: An introduction. Geological Society of America, 197, 1-18. doi:doi: 10.1130/0-8137-1197-5.1

Trade India. (2015, November 13). Retrieved from Trade India: http://pimg.tradeindia.com/01709736/b/1/Proton-PrecessionMagnetometer.jpg

Transport Canada. (2015, November 13). Flying an unmanned aircraft recreationally. Retrieved from Transport Canada - Government of Canada: http://www.tc.gc.ca/eng/civilaviation/standards/general-recavi-uav2265.htm?WT.mc_id=21zwi

Ugalde, H., L'Heureux, E., \& Milkereit, B. (2007). An integrated geophysical study for orebody delineation, Nash Creek, New Brunswick. Proceedings of Exploration 07: Fifth Decennial International Conference on Mineral Exploration, (pp. 1055-1058).

United States Geological Survey. (2015, November 6). Retrieved from USGS: http://pubs.usgs.gov/sm/mag_map/

Wang, B. (2006). 2D and 3D potential-filed upward continuation using splines. Geophysical Prospecting, 54, 199-209.

Wikipedia. (2015, November 13). Retrieved from Wikipedia: https://commons.wikimedia.org/wiki/File:Floating_core_fluxgate_inclinome ter_compass_autonnic.jpg

Williams, P. (1993). Aeromagnetic compensation using neural networks. Neural Computing and Applications, 207-214. 
Wood, A., Cook, I., Doyle, B., Cunningham, M., \& Samson, C. (2016). Experimental aeromagnetic survey using an unmanned air system. The Leading Edge - In Press. 


\section{Appendix A - Derivation of gradiometry error propagation}

The derivation for the error on magnetic gradient, as discussed in Section 3.1.3, is shown below.

Using the magnetic gradient equation,

$$
\frac{\Delta B}{\Delta x}=\frac{B_{1}-B_{2}}{x_{1}-x_{2}}
$$

$$
\frac{\Delta B}{\Delta x}=\frac{B_{1}-B_{2}}{\Delta x}
$$

where $\frac{\Delta B}{\Delta x}$ is the magnetic gradient, $B_{1}$ and $B_{2}$ are magnetic field measurements from two magnetometers separated by a distance $\Delta x$ (or $x_{1}-x_{2}$ ).

The error on a function, $f(x, y, \ldots)$, is determined following the standard error propagation equation (Eq. A.3). Where $\delta x$ and $\delta y$ are the errors on variables $x$ and $y$.

$$
\delta f(x, y, \ldots)=\sqrt{\left(\frac{\partial f(x, y, \ldots)}{\partial x}\right)^{2} \delta x^{2}+\left(\frac{\partial f(x, y, \ldots)}{\partial y}\right)^{2} \delta y^{2}+\cdots} \quad \text { Eq. A.3 }
$$

By substituting the magnetic gradient (Eq. A.2) into Eq. A.3, the gradiometry error can be determined. 


$$
\begin{gathered}
\delta \frac{\Delta B}{\Delta x} \\
=\sqrt{\left(\frac{\partial\left(\frac{B_{1}-B_{2}}{\Delta x}\right)}{\partial B_{1}}\right)^{2} \delta B_{1}{ }^{2}+\left(\frac{\partial\left(\frac{B_{1}-B_{2}}{\Delta x}\right)}{\partial B_{2}}\right)^{2} \delta B_{2}{ }^{2}+\left(\frac{\partial\left(\frac{B_{1}-B_{2}}{\Delta x}\right)}{\partial \Delta x}\right)^{2} \delta \Delta x^{2}} \text { Eq. A.4 } \\
\delta \frac{\Delta B}{\Delta x}=\sqrt{\delta B_{1}{ }^{2}+\delta B_{2}{ }^{2}+\left(\frac{1}{\Delta x^{2}}\right)^{2} \delta \Delta x^{2}} \quad \text { Eq. A.4 }
\end{gathered}
$$

The recording errors on each magnetometer are $\delta B_{1}$ and $\delta B_{2}$ and are equivalent to each other $\left(\delta B=\delta B_{1}=\delta B_{2}\right)$. The error on magnetometer separation is $\delta \Delta x$.

The effects on the gradiometry error due to magnetometer separation is considered negligible. This approximation is valid due to the fact that the separation distance is fairly large (ranging from $5 \mathrm{~m}$ to $20 \mathrm{~m}$ ) and that the magnetometers are rigidly mounted on the aircraft. As a result, the separation error is small, on the order of centimeters. This results in the gradiometry error equation becoming that shown in Eq. A.5:

$$
\delta \frac{\Delta B}{\Delta x}=\sqrt{2} \frac{\delta B}{\Delta x}
$$




\section{Appendix B - First derivative of the total magnetic field}

As used in Section 3.1.4 and in Eq. 3.13, the first derivative of the total magnetic field is:

$$
\frac{d}{d x}\left(B_{T}\right)=\frac{B_{x} \frac{d}{d x}\left(B_{x}\right)+B_{y} \frac{d}{d x}\left(B_{y}\right)+B_{z} \frac{d}{d x}\left(B_{z}\right)}{\sqrt{B_{x}^{2}+B_{y}^{2}+B_{z}^{2}}}
$$

where the derivatives of $B_{x}, B_{y}$, and $B_{z}$ are as follows:

$$
\begin{aligned}
\frac{d}{d x}\left(B_{x}\right)= & \frac{4 H_{o} \sin (\beta) x-3 H_{o} \cos (\beta) y+3 Z_{o} z}{\left(x^{2}+y^{2}+z^{2}\right)^{5 / 2}} \\
& -\frac{5 x\left(H_{o} \sin (\beta)\left(2 x^{2}-y^{2}-z^{2}\right)-3 H_{o} \cos (\beta) y x+3 Z_{o} z x\right)}{\left(x^{2}+y^{2}+z^{2}\right)^{7 / 2}} \\
\frac{d}{d x}\left(B_{y}\right)= & \frac{3 H_{o} \sin (\beta) y+2 H_{o} \cos (\beta) x}{\left(x^{2}+y^{2}+z^{2}\right)^{5 / 2}} \\
& -\frac{5 x\left(3 H_{o} \sin (\beta) y x-H_{o} \cos (\beta)\left(-x^{2}+2 y^{2}-z^{2}\right)+3 Z_{o} z y\right)}{\left(x^{2}+y^{2}+z^{2}\right)^{7 / 2}} \\
\frac{d}{d x}\left(B_{z}\right)= & \frac{3 H_{o} \sin (\beta) z-2 Z_{o} x}{\left(x^{2}+y^{2}+z^{2}\right)^{5 / 2}} \\
& -\frac{5 x\left(3 H_{o} \sin (\beta) z x-3 H_{o} \cos (\beta) y z+Z_{o}\left(-x^{2}-y^{2}+2 z^{2}\right)\right)}{\left(x^{2}+y^{2}+z^{2}\right)^{7 / 2}}
\end{aligned}
$$

Eq. B.3

Eq. B.4 


\section{Appendix C - Minimum detection capability surface plots}

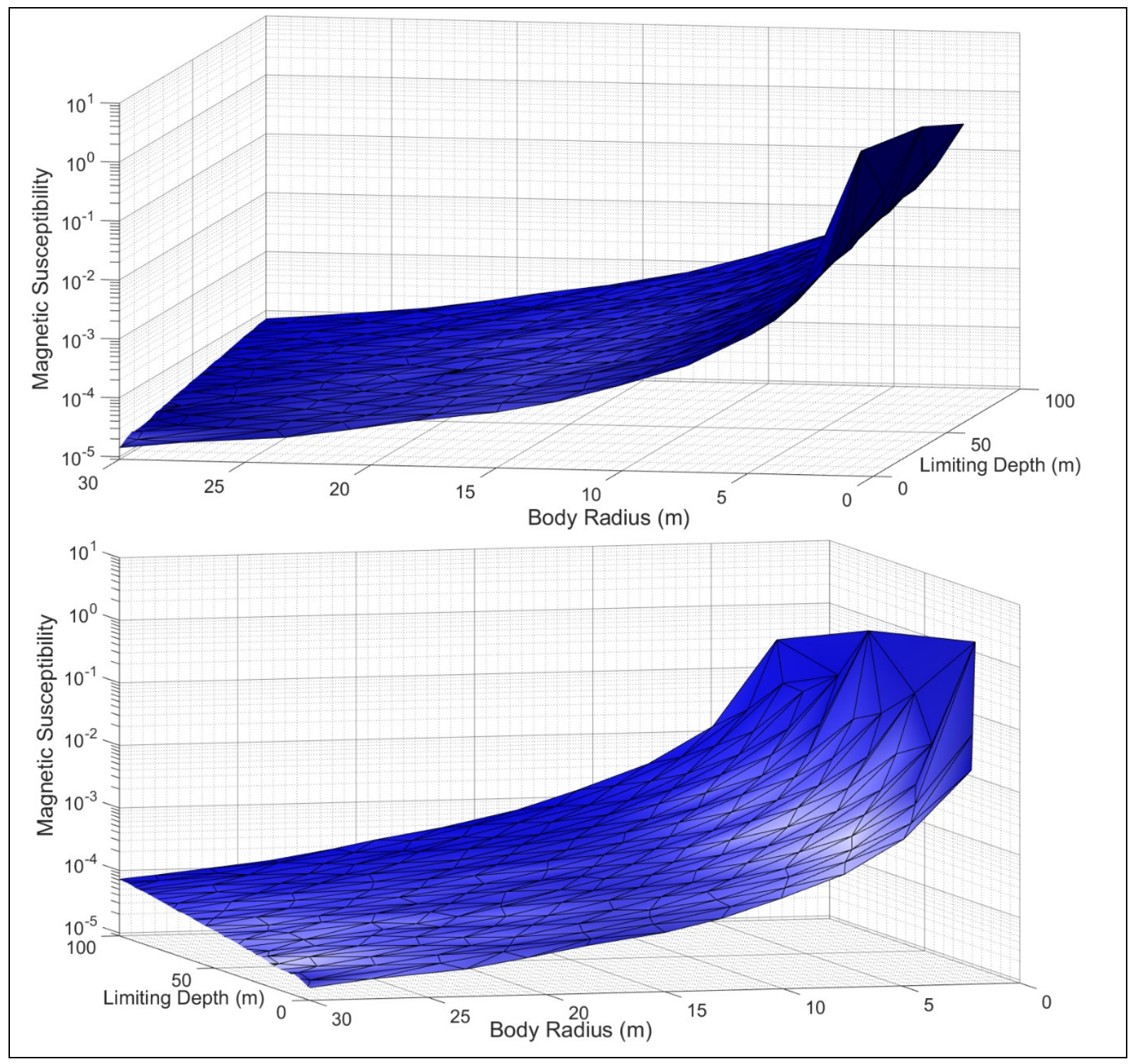

Figure C.1: Surface plot of the minimum detection limits with a 90\% success rate for a flight altitude of $100 \mathrm{~m}$ (i.e. the Traditional Fixed-wing system). Two views of the same plot. 


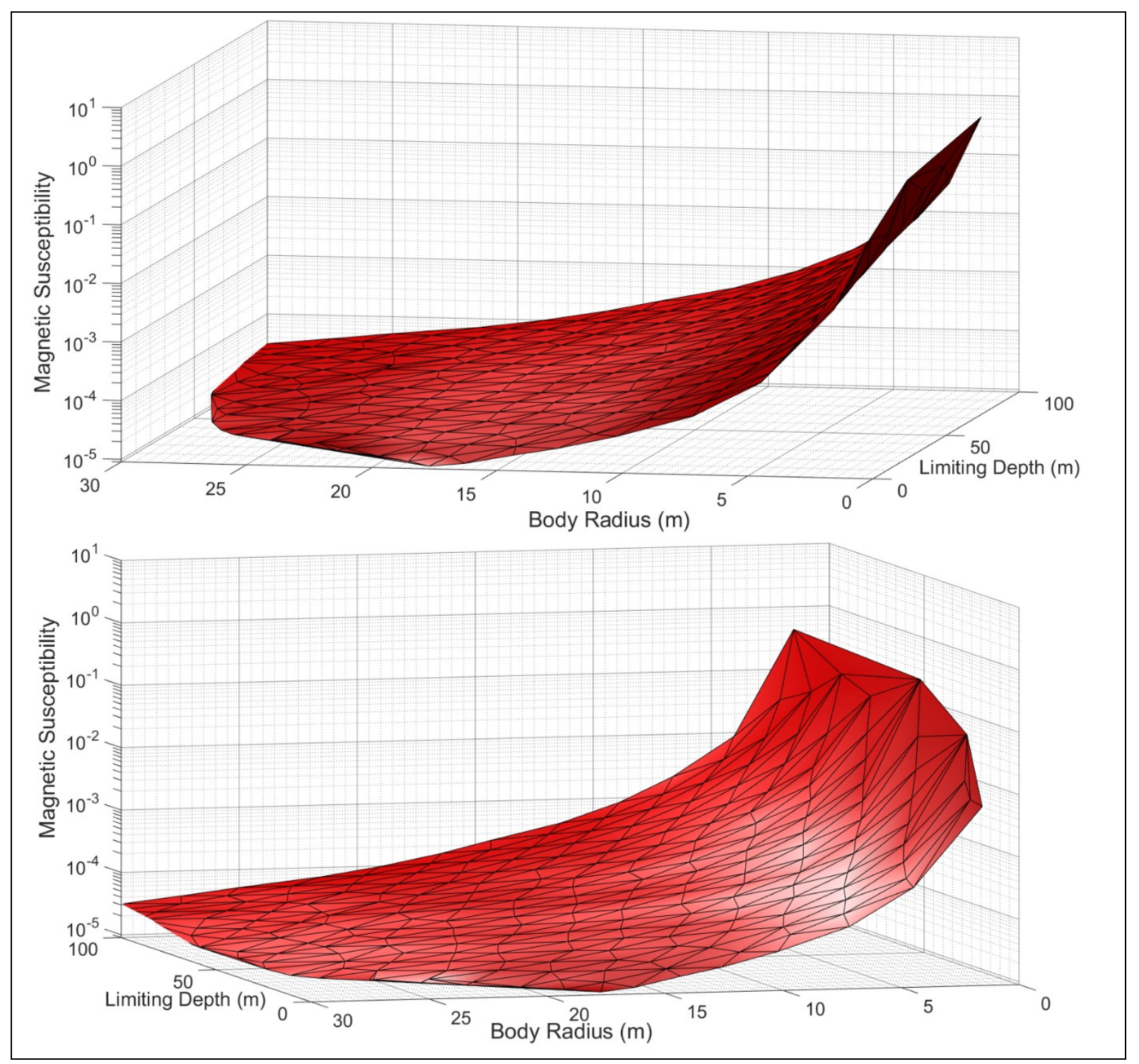

Figure C.2: Surface plot of the minimum detection limits with a $90 \%$ success rate for a flight altitude of 50m (i.e. the Venturer UAS). Two views of the same plot. 


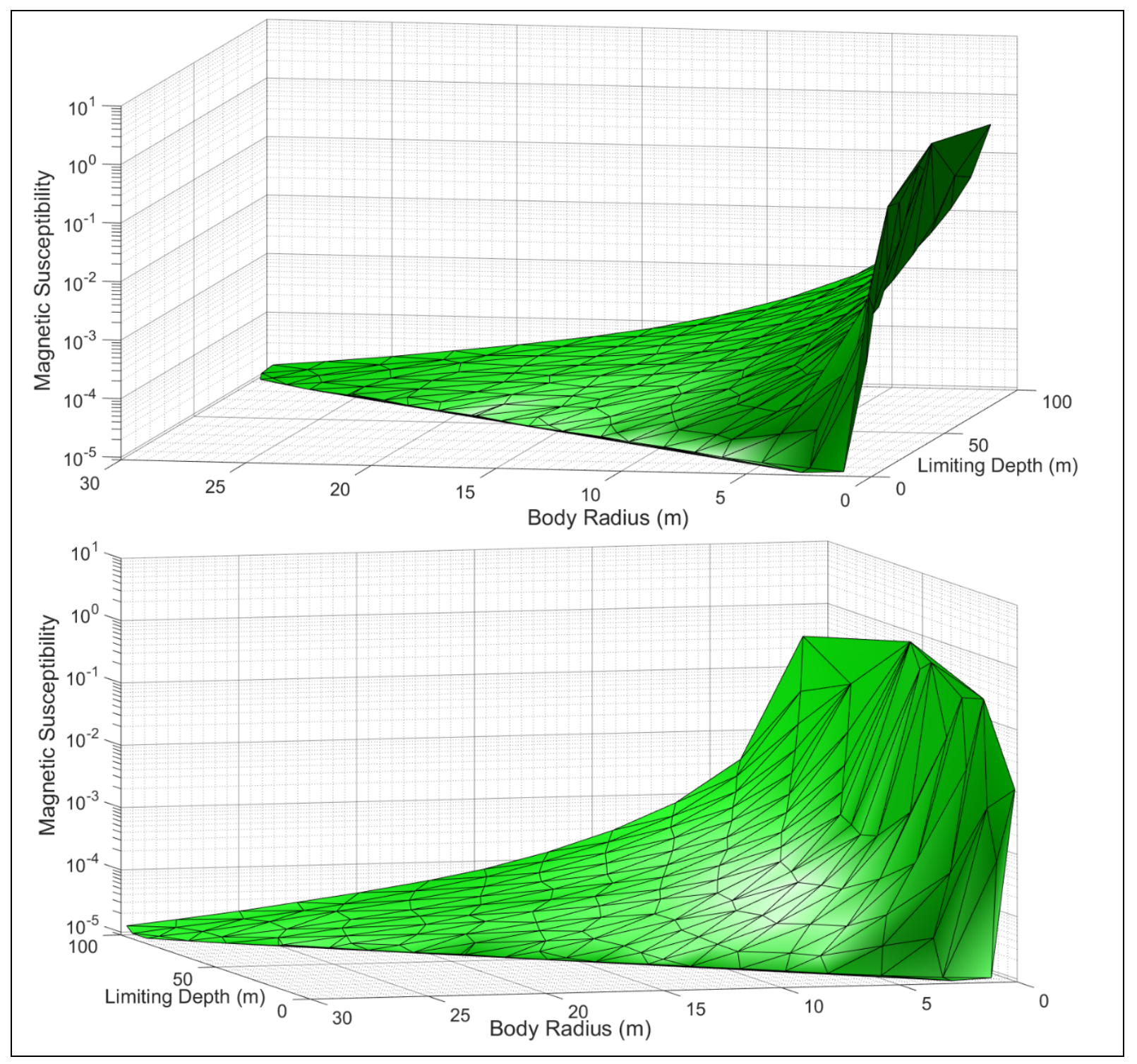

Figure C.3: Surface plot of the minimum detection limits with a 90\% success rate for a flight altitude of $2 m$ (i.e. the SkyLance UAS). Two views of the same plot. 